\title{
ॠUSGS
}

science for a changing world

\section{Benthic Response to Water Quality and Biotic Pressures in Lower South San Francisco Bay, Alviso Slough, and Coyote Creek}

By Francis Parchaso, Janet K. Thompson, Jeff S. Crauder, Rosa I. Anduaga, and Sarah A. Pearson

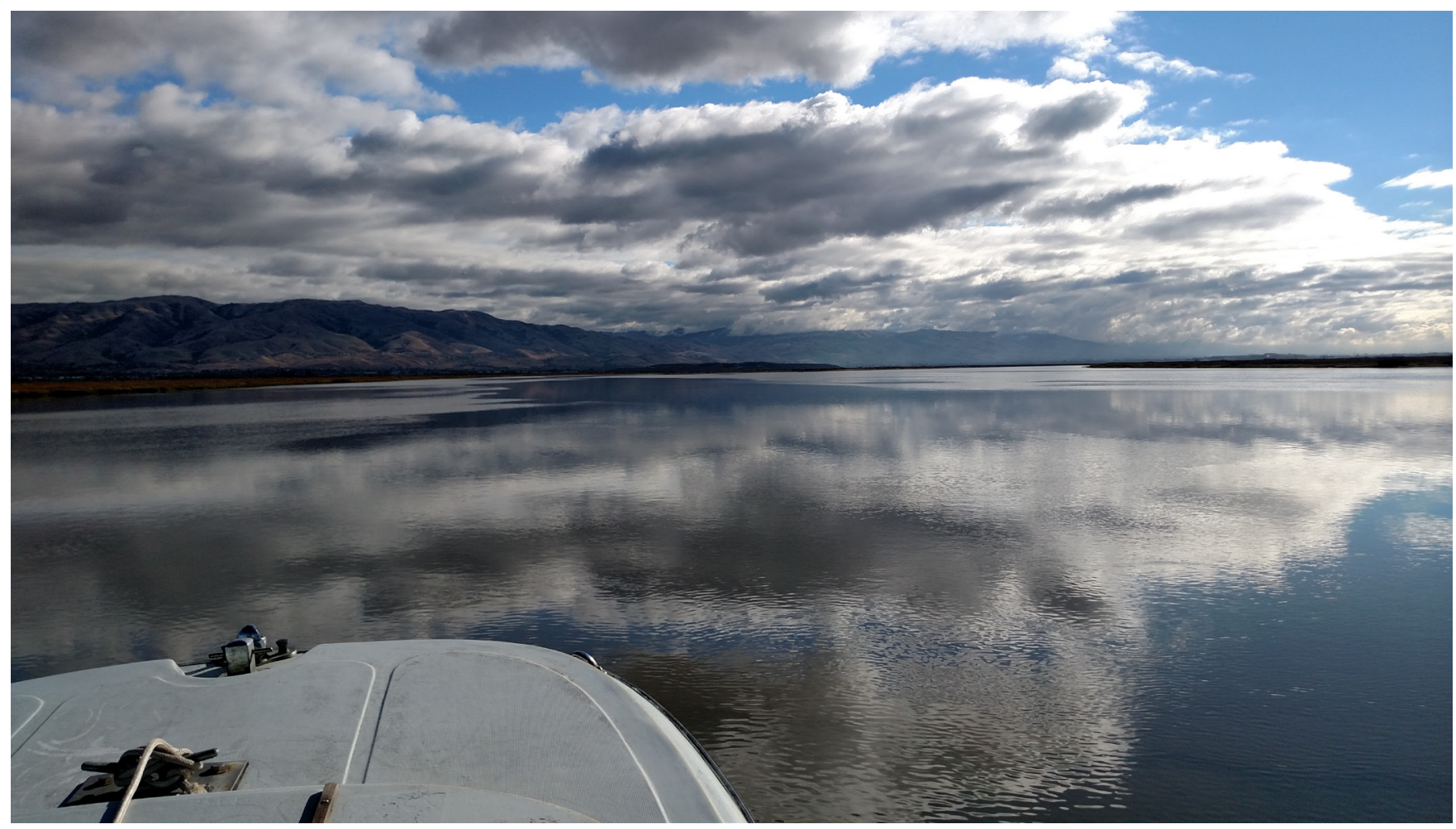

Open-File Report 2015-1234

U.S. Department of the Interior

U.S. Geological Survey 


\section{U.S. Department of the Interior \\ SALLY JEWELL, Secretary}

\section{U.S. Geological Survey \\ Suzette M. Kimball, Acting Director}

U.S. Geological Survey, Reston, Virginia: 2015

For more information on the USGS-the Federal source for science about the Earth, its natural and living resources, natural hazards, and the environment-visit http://www.usgs.gov/ or call 1-888-ASK-USGS (1-888-275-8747).

For an overview of USGS information products, including maps, imagery, and publications, visit http://www.usgs.gov/pubprod/.

Any use of trade, firm, or product names is for descriptive purposes only and does not imply endorsement by the U.S. Government.

Although this information product, for the most part, is in the public domain, it also may contain copyrighted materials as noted in the text. Permission to reproduce copyrighted items must be secured from the copyright owner.

Suggested citation:

Parchaso, F., Thompson, J.K., Crauder, J.S., Anduaga, R.I., and Pearson, S.A., 2015, Benthic response to water quality and biotic pressures in lower south San Francisco Bay, Alviso Slough, and Coyote Creek: U.S. Geological Survey Open-File Report 2015-1234, 44 p., http://dx.doi.org/10.3133/ofr20151234.

ISSN 2331-1258 (online) 


\section{Contents}

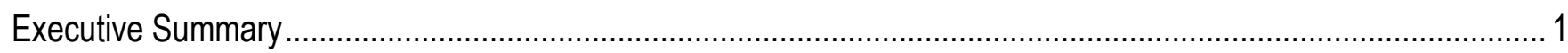

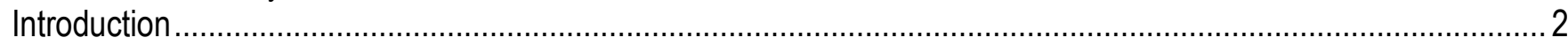

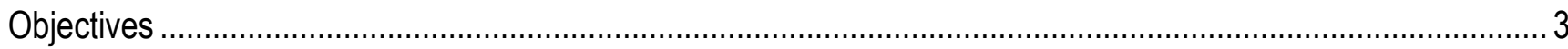

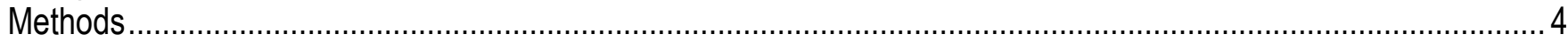

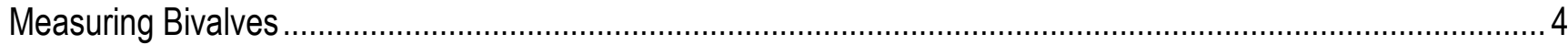

Biomass Calculations ....................................................................................................................... 4

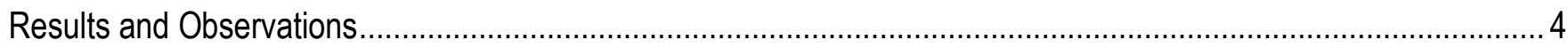

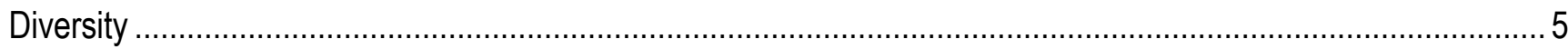

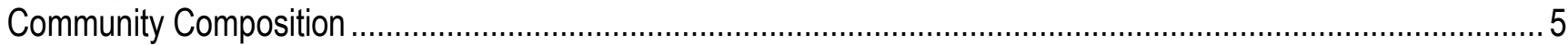

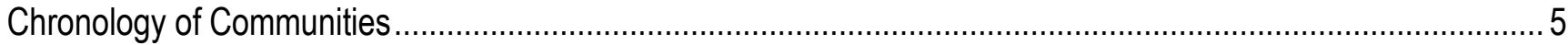

Transitions in Benthic Community Function-As Consumers, As Prey, As Geochemical Enhancers .....................6

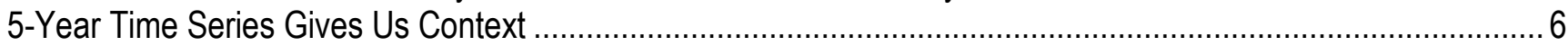

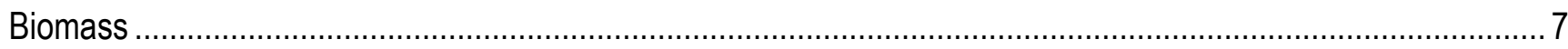

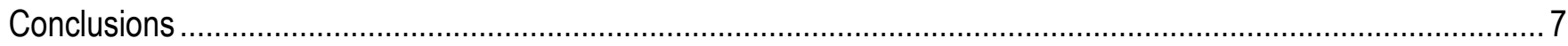

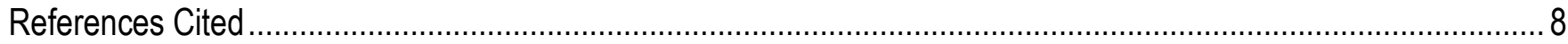

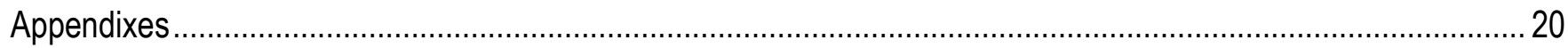

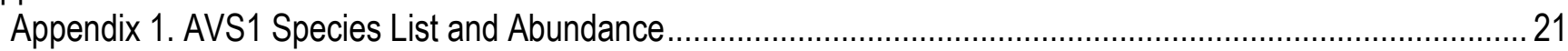

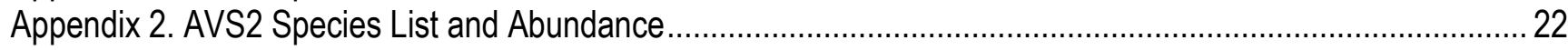

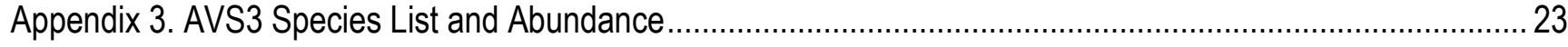

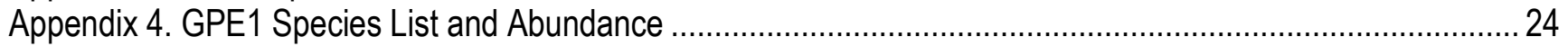

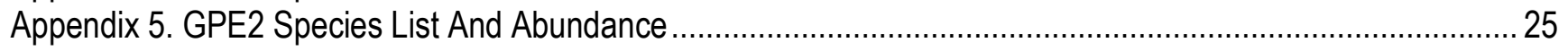

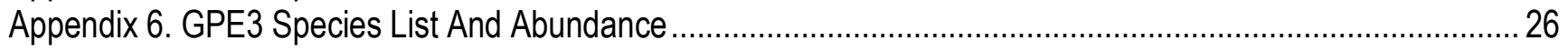

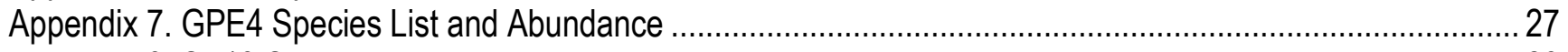

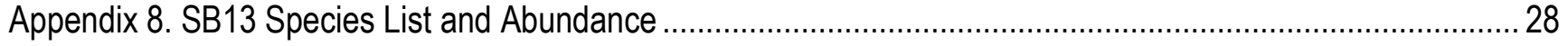

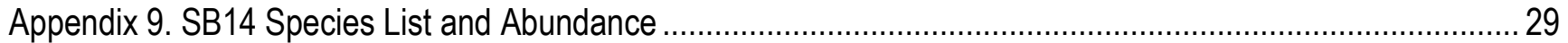

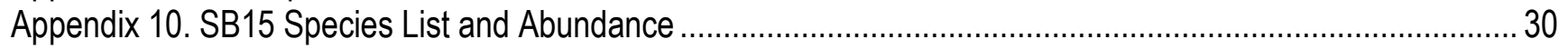

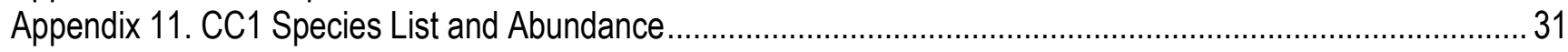

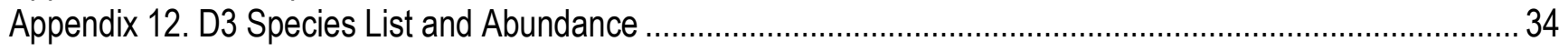

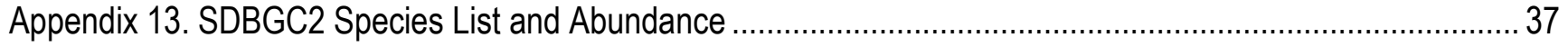

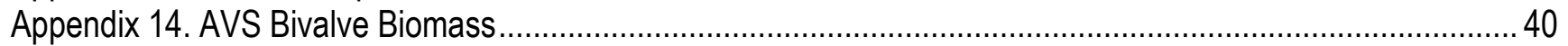

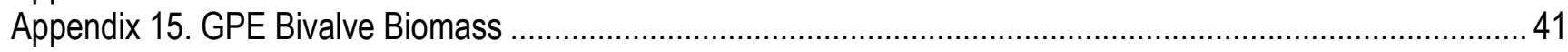

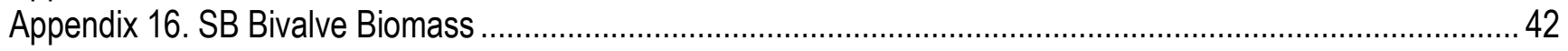

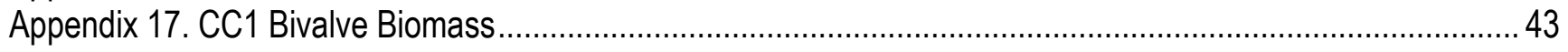

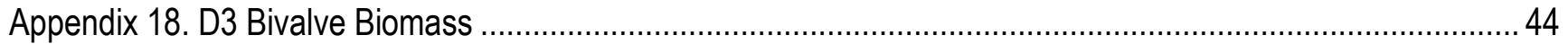

\section{Figures}

1. Map showing benthic sampling station locations in south San Francisco Bay and sampling area in relaton to San Francisco Bay proper. ……............................................................................................. 10

2. Maps showing the number of species at each station in south San Francisco Bay and its adjacent sloughs for March, June, and September 2014.

3. Map showing abundance of all species at all stations in March 2014 (for species where the abundance is greater than 2 per 0.05 square meter). 
4. Map showing abundance of all species at all stations in June 2014 for species where the abundance is greater than 2 per 0.05 square meter). 12

5. Map showing abundance of all species at all stations in September 2014 (for species where the abundance

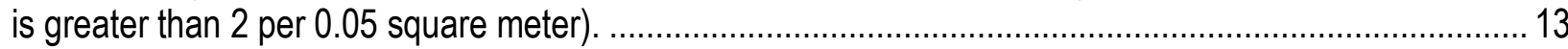

6. Time series graph showing abundance of class Bivalvia at station D3 in Coyote Creek, 2009-2014 ........ 14

7. Time series graph showing abundance of Order Amphipoda at station D3 in Coyote Creek, 2009-2014 ... 14

8. Time series graph showing abundance of class Bivalvia at station CC1 in Coyote Creek, 2009-2014 ...... 15

9. Time series graph showing abundance of Order Amphipoda at station CC1 in Coyote Creek, 2009-2014 15

10. Map showing bivalve biomass at sampling stations in Guadalupe Slough, Alviso Slough, Artisean Slough, and Coyote Creek in March 2014.

11. Map showing bivalve biomass at sampling stations in Guadalupe Slough, Alviso Slough, Artisean Slough, and Coyote Creek in June 2014.

12. Map showing bivalve biomass at sampling stations in Guadalupe Slough, Alviso Slough, Artisean Slough, and Coyote Creek in September 2014. 17

13. Time series graph showing bivalve biomass at station D3 in Coyote Creek, 2009-2014 ............................... 18

14. Time series graph showing bivalve biomass at station CC1 in Coyote Creek, 2009-2014 ........................ 18

\section{Tables}

1. Benthic sampling station coordinates (latitude-longitude). 


\title{
Benthic Response to Water Quality and Biotic Pressures in Lower South San Francisco Bay, Alviso Slough, and Coyote Creek
}

\author{
By Francis Parchaso, Janet K. Thompson, Jeff S. Crauder, Rosa I. Anduaga, and Sarah A. Pearson
}

\section{Executive Summary}

Benthic invertebrate communities are monitored because the composition of those communities can effect and be affected by the water quality of an aquatic system. Benthic communities use and sometimes regulate the cycling of essential elements (for example, carbon). Benthic invertebrate taxa may also indicate acutely and chronically stressful environments because they are mostly sessile, accumulate contaminants, and sometimes respond dramatically to oligotrophic as well as eutrophic conditions. Benthic communities can in turn affect water quality by grazing pelagic food resources and increasing the rate of nutrient regeneration through feeding and bioturbating the sediment.

South San Francisco Bay is a system dependent on phytoplankton as the base to the food web. Despite abundant nutrients, south San Francisco Bay has had limited phytoplankton production in the last several decades owning to poor light conditions and high grazing losses from the water column by benthic invertebrates. The south San Francisco Bay achieves a balance of biogeochemical conditions in most springs to accommodate a short phytoplankton bloom. This balance has maintained the phytoplankton in south San Francisco Bay at low biomass levels relative to other high-nutrient urban estuaries. The role that benthic invertebrates play in this balance, in these episodic spring events, and in other seasons within the estuary remains of great interest to water-quality and biological resource managers.

Our primary objective in this study is to quantify current (2014) benthic-community structure and function in the south San Francisco Bay sloughs and to compare those communities temporally over decadal time scales with a unique long-term dataset. The study area (fig. 1) is inclusive of the area south of the Dumbarton Bridge (DB) including Alviso and Guadalupe Sloughs and Coyote Creek.

The following are results highlighted in this report:

- The benthic communities of Coyote Creek and Alviso, Guadalupe, and Artesian Sloughs were dominated by different organisms but similar functional groups in March, June, and September 2014.

- Coyote Creek stations (D3 and CC1) had a similar increase in Potamocorbula amurensis biomass followed by a decline in 2014, as was observed annually in the south San Francisco Bay since 1999. The lack of grazing pressure owing to the observed declines in these taxa in 2014 may allow the sloughs to become sources of phytoplankton in spring.

- Bivalve biomass is elevated in summer and fall relative to the spring and winter except in Artesian Slough, where bivalves did not establish a signifcant presence. Presence of certain species contributes to the prey value of the community to predators. Potamocorbula amurensis 
is a shallow-burrowing bivalve and hence is easy prey for bottom-feeding predators. In contrast, Macoma petalum is a deposit feeder and can burrow deeper into the substrate than Potamocorbula amurensis, making it harder to be preyed upon. The quantitative importance of such predator-prey relationships on phytoplankton dynamics requires further investigation. There were also more amphipods in the sloughs in March 2014; this group is another potential contributor to the benthic-pelagic biomass balance. There is no observed reason for Artesian Slough to have low bivalve biomass values and high amphipod abundances.

\section{Introduction}

Benthic communities are monitored because the individuals reflect the water quality of a system and because they use and sometimes control available carbon resources. They are also a good monitor of acutely and chronically stressful environments because they are stationary, accumulate contaminants, and respond, sometimes dramatically, to low and high phytoplankton biomass as well as low oxygen conditions.

Benthic communities can affect water quality by grazing pelagic food resources and increasing the rate of nutrient regeneration through feeding and bioturbating the sediment. South San Francisco Bay is a system dependent on phytoplankton as the base to the food web (Jassby and others, 1993). Despite abundant nutrients, south San Francisco Bay has had limited phytoplankton production in the last several decades owing to poor light conditions and high grazing losses. Thus, the system has rarely experienced anoxia that is usually associated with high nutrient systems (Cloern, 2001). Our conceptual model for phytoplankton growth in south San Francisco Bay includes a delicate balance between light availability, grazing losses (primarily in the shallow water), and physical mixing of the water column (Lucas and others, 2009). The south San Francisco Bay achieves the right balance in most springs to accommodate a short phytoplankton bloom. This balance has maintained the phytoplankton in south San Francisco Bay at low biomass levels relative to other high-nutrient urban estuaries (Cloern, 2001).

We have found that a fall/winter reduction in filter-feeding bivalves in the shallow water each year is one of the primary triggers that allow a spring phytoplankton bloom to develop in the south San Francisco Bay. Each fall, predation by migratory and resident birds (Thompson and others, 2008), fish, and invertebrates (Cloern and others, 2007) decimates the shallow water bivalve communities in both south San Francisco and San Pablo Bays (Poulton and others, 2002, 2004; Richman and Lovvorn, 2004). This elimination of bivalve grazing in the shallow water allows the phytoplankton to grow if light and mixing are not limiting (Thompson and others, 2008). Bivalves in the shallow water are thus essentially an annual species with larvae settling each spring followed by rapid growth, which allows them to become a controlling factor on the phytoplankton by late spring and summer.

Changes in the benthic community structure occur normally. Benthic species distributions are dependent on the physical habitat (substrate and depth), physiological limits (such as salinity in this system; Lee and others, 2003), and predators (Cloern and others, 2007). Therefore, seasonal and interannual differences in freshwater flow result in both seasonal and episodic changes in species abundance and community composition (Nichols and Thompson, 1985a, 1985b). Episodic events such as the introduction of introduced species can have lasting effects on the benthic community, whereas other events such as wastewater treatment plant malfunctions are likely to have short-term effects. Contaminants can also restrict the success of some species (Hornberger and others, 2000). Therefore, when the benthic community that was dominated by filter feeders changes to one dominated by surface deposit feeders, many possible factors could be responsible for such a change.

Our current hypothesis is that a change in predator abundance associated with upwelling off

shore has reduced all bivalves in south San Francisco Bay. The result of the reduced grazing pressure on 
the phytoplankton in conjunction with decreased turbidity lead an increasing trend in phytoplankton biomass and the development of fall increases in phytoplankton biomass (Cloern and others, 2007). As shown by this example, understanding the ecological dynamics of the south San Francisco Bay is not always straightforward. This is a cautionary story, as it is important that we not misinterpret changes in phytoplankton standing stock to be due to the changing water-quality conditions when they are really due to biotic changes.

We know how important predation on the benthic bivalves can be, but the benthic invertebrates are also important as prey. Benthic invertebrates - a significant prey resource for many fish speciesare considered a component of essential fish habitat (Fisheries Management Plans under the Magnuson Stevens Fishery Conservation and Management Act). Our conceptual model for maintaining appropriate benthic prey for fish and bird species is based on understanding what prey characteristics and habitat of the prey are important for the predator. The effect of species swaps within benthic communities such as we have seen in the last 15 years may be very significant to predators. For example, a surface-dwelling bivalve like Potamocorbula amurensis has a soft shell, is highly caloric, is easy to capture, and has been shown to be valuable prey in San Francisco Bay (Richman and Lovvorn, 2004). A deep-burrowing, tube-dwelling worm such as Sabaco elongates, which is common in south San Francisco Bay (Lee and others, 2003), is unlikely to be fed upon by either fish or birds. For this reason, analysis of the benthic community needs to include abundance, grazing rate where appropriate, and functional ecology (feeding mode, habitat, motility, and structures such as tubes and shells, which may impede predation) of each species.

\section{Objectives}

Our primary objective is to investigate the current community structure and function in the benthic community in the sloughs connected to south San Francisco Bay and to compare those communities as well as the benthic community in the south San Francisco Bay. The study (fig. 1) is inclusive of the area south of the Dumbarton Bridge (DB), including Alviso and Guadalupe Sloughs and Coyote Creek. If possible, stations upstream of the railroad bridge on Coyote Creek will be included. A number of channel stations are included because the bivalves in the channel are the source of recruits following the fall predation on the bivalves. Thus, to fully understand the benthic community changes through the seasons, we need benthic community data from all depths.

The abundance of filter-feeding benthic organisms in south San Francisco Bay has declined in the past decade (Cloern and others, 2007). We ask if the benthic communities are similar in the lower south San Francisco Bay sloughs and south San Francisco Bay and if the sloughs could serve as a source of filter feeders to south San Francisco Bay?

The hypotheses tested by this study include the following:

- $\mathrm{H}_{0}$ : There is a difference in the structure of benthic communities in south San Francisco Bay and in the adjacent sloughs.

- $\mathrm{H}_{0}$ : The differences in the benthic community structure between south San Franisco Bay and the adjacent sloughs and among sloughs cannot be associated with known stressors (predators, hydrologic events, invasive species, and water quality).

- $\mathrm{H}_{0}$ : Grazing pressure in the sloughs shows that functional differences in the benthic community in the sloughs relative to south San Francisco Bay have little influence on south San Francisco Bay phytoplankton biomass.

- $\mathrm{H}_{0}$ : Differences in the benthic community structure of the south San Francisco Bay sloughs and the south San Francisco Bay result in an increase in the food available to predators in the sloughs either through changes in biomass and availability or palatability of benthic organisms. 


\section{Methods}

Analysis of the species composition and functional composition of the benthic community incorporates previously collected samples and samples collected in 2014. Samples that were previously collected are from 2009 to 2013 as part of a field and modeling study that examined the mechanisms of phytoplankton bloom development in south San Francisco Bay (Thompson and others, 2008; Lucas and others, 2009). These samples are archived at the U.S. Geological Survey (USGS).

Samples collected in 2014 from Alviso, Artesian, and Guadalupe Sloughs were collected during the months of March, June, and September to coincide with our spatially intensive sampling of south San Francisco Bay below the San Mateo Bridge (table 1). The remaining stations in south San Francisco Bay (D3, SDBGC2, and CC1) were collected monthly (fig. 1).

Samples were collected with a $0.05-\mathrm{m}^{2}$ (square meters) weighted Van Veen grab that was hand deployed in all but the deep water stations. Samples were sieved through a $0.5-\mathrm{mm}$ (millimeter) screen, preserved in 10-percent buffered formalin, and transferred to 70-percent ethyl alcohol with rose bengal dye. Samples were sorted and well-known species enumerated at the USGS Benthic Lab in Menlo Park. A quality assurance procedure was used whereby, depending on the difficulty and the number of organisms in the sample, samples were double sorted/identified. More difficult taxonomic groups were contracted out to a taxonomic consultant (Susan McCormick) with a request that all but the rare species be identified and enumerated to the lowest taxon possible. The contractor was asked to supply a taxonomic voucher collection.

\section{Measuring Bivalves}

U.S. Geological Survey personnel measured the bivalves to the nearest $\mathrm{mm}$ using a video image analyzer with HL++ image software (Western Vision, 2005) and handheld calipers (for the larger animals). Data were saved in size frequency spreadsheets. The USGS has used this technique for over 20 years. Large bivalves were measured for conversion to biomass in the recent samples (2004-08) to allow for comparison with previous data (1993-2009).

\section{Biomass Calculations}

All filter-feeding bivalve individuals (Potamocorbula amurensis, Venerupis japonica, Musculista senhousia, Mytilus c.f. edulis, Mya arenaria, and Macoma petalum) were removed from each sample and counted, and the longest length of each individual was measured. A size range (length of $1.5 \mathrm{~mm}$ and greater) of live animals was collected at each site during each month to calculate dry tissue weight for each size of animal. Animals were measured, dried at $60{ }^{\circ} \mathrm{C}$ (dry weight), weighed, ashed at $500{ }^{\circ} \mathrm{C}$ in a muffle furnace, and reweighed (ash weight). A relationship between animal length and ash-free dry weight (AFDW $=$ dry wt. - ash wt.) was then used to convert the measured animals from each benthic sample into biomass estimates for each species.

\section{Results and Observations}

We look at several components of the benthic community to help us interpret its response to water-quality conditions and to characterize its function within the ecosytem. We first look at the relative diversity of the communities, which reflects the degree and frequency of disturbance, both natural and human caused. Our second task is to examine the composition of the communities, which when combined with assignment of functional traits of the species (feeding mode, reproductive mode, and life history), tells us how the species in the community function as prey, predator, and facilitator of 
geochemical processes. It is the combination of these factors at several locations, ideally oriented along a gradient, that tell us the most.

\section{Diversity}

We show here the simplest index of diversity - the number of species found at each station for each month sampled in 2014. The general pattern was consistent among the seasons (fig. 2). The highest diversity was seen in the south San Francisco Bay transect (stations SBDCG2, CC1, and D3), where species number ranges from 6 to 12. Guadalupe Slough and Alviso Slough had similar diversities (4-10 species), and Artesian Slough consistently had the lowest diversity with a range of 1 to 6 species. It was also the only slough where the down-bay (closest to the bay) station did not have the most species on the transect. With a few exceptions, the diversity was highest in June and lowest in September 2014.

\section{Community Composition}

Bivalves and amphipods were the dominant taxonomic groups in Alviso Slough, Guadalupe Slough, Artesian Slough, Coyote Creek, and south San Francisco Bay stations during our 2014 sampling (figs. 3-5). The most common amphipods are tube-dwelling filter feeders. They are oviparous/viviparous (brood their young) and release their young as juveniles. Because most amphipods with these traits can swim, rapidly leave an area when challenged, and rapidly invade an area to reestablish their tubes and burrows, they are considered to be opportunistic species in spite of producing a relatively low number of juveniles. They feed on pelagic food sources (phytoplankton is common), surface detritus, and bacteria. The high number of individuals at some locations result in the amphipods consuming a noticeable amount of the pelagic food, but in most cases, they still have an order of magnitude or less affect on the phytoplankton biomass relative to the bivalves. Amphipods are common prey items for fish and birds (shore birds and ducks). Both pelagic and demersal fish feed on amphipods as some species vacate their tubes at night and actively swim, making them available to pelagic feeders.

Bivalves in the south San Francisco Bay and adjacent sloughs are filter feeders or deposit feeders with one species being capable of feeding both ways. They are broadcast spawners with external fertilization except for Gemma gemma, which broods its young. Except for small surface-dwelling species (Gemma gemma and juvenile Potamocorbula amurensis), bivalves are usually stationary once they settle. The large size of bivalves relative to the rest of the benthic community means they consume a significant amount of the available carbon in the water column and in the sediment surface. Their large size also allows them to "wait out" periods of unfavorable water quality and low food availability and respond very quickly once conditions change. Therefore, they are formidable competitors with pelagic filter feeders such as zooplankton, which must reproduce and grow populations in response to increased food. Bivalves are important prey for demersal fish and birds.

The annelids were remarkably absent in our sampling. Of the annelid species that did commonly occur, most were subsurface-deposit feeders (Neanthes succinea, Heteromastus filliformis, and members of the tubificidae family), eating through the sediment in much the same way that earthworms feed.

\section{Chronology of Communities}

The March 2014 sampling was dominated by amphipods except at AVS1 and D3. Species diversity was highest in March and similar at Alviso and Guadalupe Sloughs, although the abundances were considerably higher in Alviso Slough than in Guadalupe Slough. Bivalve abundance was higher at stations where amphipod abundance was low; both of these sloughs had a combination of Macoma 
petalum, a filter-feeding/deposit-feeding bivalve, and Corophidae amphipods. Artesian Slough had the lowest diversity and the greatest number of individuals, which were mostly the amphipod

Monocorophium. Corophium, a member of the same amphipod family as Monocorophium, was the dominant amphipod in the more estuarine south San Francisco Bay station SDBGC2. Gemma gemma, a tiny (maximum 5-mm) bivalve, was the dominant species at D3 and AVS1, the stations near the convergence of Coyote Creek and south San Francisco Bay.

The stations with the greatest number of indiviuals shifted from Artesian Slough to the upper Guadalupe Slough (GPE3) and south San Francisco Bay (SDBGC2 and D3) stations in June. Along with the spatial shift in dominant stations, there was a change in the dominant species. All three south San Francisco Bay stations were dominated by Gemma gemma. Monocorophium continued to be the dominant species in the upper slough stations, where it was joined by Macoma petalum and Potamocorbula amurensis. Species diversity continued to be greatest in the lower Guadalupe and Alviso Sloughs in addition to the south San Francisco Bay stations.

More transitions in the benthic community were observed in the September sampling. There was an increase in the number of invidiuals at the most upstream locations in Alviso and Guadalupe Sloughs. The total number of individuals in south San Francisco Bay stations declined from the June to September sampling with Gemma gemma being replaced as the dominant species by Ampelisca abdita, a tube-dwelling, filter-feeding amphipod. The increase in abundance of animals from June to September in the upper sloughs was due to increases in the abundance of Monocorophium.

\section{Transitions in Benthic Community Function-As Consumers, As Prey, As Geochemical Enhancers}

Filter feeders are effective at moving carbon from the water column to the benthos both as food and as feces, so their presence can speed up the geochemical processing of organic matter. For the entire sampling period, filter feeders were the dominant feeding type present at our sites. Filter feeders are also capable of grazing down phytoplankton biomass, and the grazing pattern that we observed with the bivalves in 2014 is similar to the pattern that we have observed with bivalves in south San Francisco over the last 30 years. The bivalves were absent in March, increased in June, were an integral part of the phytoplankton growth dynamics in summer, and then disappeared in fall. We assume that the same predators that consume San Francisco Bay bivalves were at least partially responsible for the decline in bivalve abundance in all the south San Francisco Bay sloughs in fall. This pattern of decreased numbers of bivalves in the spring followed with increasing numbers by summer is a pattern that is observed in the greater south San Francisco Bay.

The amphipods were present all year but responded to the changes in bivalve presence quickly, as seen by the shifting relative dominance of amphipods from Artesian Slough in March to upper Guadalupe Slough in June and then to the upper reaches of Guadalupe and Alviso Sloughs in September. The change in amphipod abundance may have been due to them relocating or being preyed upon, but they were still the most abundant group at all stations in September.

\section{5-Year Time Series Gives Us Context}

Time series data from stations D3 and CC1 show the temporal transition in dominant filter feeder type from bivalves to amphipods in more detail (figs. 6-9). Bivalve abundance from 2009 to 2014 at D3 and CC1 show bivalves being the dominant taxa until late 2013. In fall 2013, the amphipod abundance increased during the period when the bivalve abundance was low. We have seen this transition in abundance occur in the bay before, and it is never clear if the amphipods were successful only because the bivalves were gone or if something made it appealing to the amphipods and coincidently unappealing to the bivalves. Macoma petalum has been reported to have an opposing cycle 
to the amphipod Ampelisca abdita in this system as well as others (Nichols and Thompson, 1985a, 1985 b), so it is not an unknown dynamic.

\section{Biomass}

Biomass is the common currency between trophic levels in the food web, so an estimation of bivalve biomass can tell us not only how much they must consume to maintain their biomass but also the mass of the food available to the predators on the bivalves. We estimated biomass for Macoma petalum, a deep-living deposit/filter feeder, and for the two filter feeders, Mya arenaria and Potamocorbula amurensis. By showing the data spatially (figs. 10, 11, and 12), we can see the relative importance of the spatial changes. Bivalve biomass is generally greater in Alviso Slough and Guadalupe Slough than at the Coyote Creek and Artesian Slough stations. Biomass in Artesian Slough is consistently lower than in the other locations for each month of sampling. Macoma is the overwhemingly dominant bivalve in the March sampling. Potamocorbula has more of a presence in the June and September sampling. Mya, while not numerically dominant, was represented in the biomass data.

Bivalve biomass (shown in figs. 10,11, and 12) is a good proxy for benthic grazing rates. The biomass distribution patterns in the study area do not differ from the observed patterns in the greater south San Francisco Bay, where we see low biomass values in March and increasing biomass values in June. The biomass values in September are generally equal to June. The largest contributor to the biomass values in March was Macoma, a deposit feeder. In June and September, Potamocorbula amurensis, a very efficient filter-feeding bivalve, contributed to a greater portion of the biomass values. This change in the species contribution to the community suggests that there may be a greater grazing pressure on the phytoplankton community in the summer and fall than in the spring. This would allow all the sloughs connected to south San Francisco Bay to be a source of phytoplankton in the spring while potentially being a phytoplankton sink in the summer and fall.

In looking at the time series data in D3 and CC1 (figs. 13 and 14), we see that there has been a decline in bivalve biomass since its peak in 2012. Also notable is the increase in Macoma and Mya biomass when Potamocorbula amurensis biomass decreases at station CC1.

\section{Conclusions}

The hypotheses tested by this study include the following:

- $\mathrm{H}_{0}$ : There is a difference in the structure of benthic communities in south San Francisco Bay and in the adjacent sloughs.

True - the benthic communities are dominated by different organisms but similar functional groups.

- $\mathrm{H}_{0}$ : The differences in the benthic community structure between south San Francisco Bay and the sloughs that connect to south San Francisco Bay and between the sloughs themselves cannot be associated with known stressors (predators, hydrologic events, invasive species, and water quality).

True - there is no observed reason for Artesian Slough to have low bivalve biomass values and high amphipod abundances. Lower salinities and predators may be limiting the presence or growth of bivalves in Artesian Slough. 
- $\mathrm{H}_{0}$ : Grazing pressure in the sloughs shows that functional differences in the benthic community in the sloughs relative to the bay have little influence on bay phytoplankton biomass.

True - stations D3 and CC1 had a similar increase in Potamocorbula amurensis biomass followed by a decline throughout 2014 sampling, as was also observed in south San Francisco Bay in 2009. The lack of grazing pressure may allow the sloughs to become sources of phytoplankton in spring.

- $\mathrm{H}_{0}$ : Differences in the benthic community structure of the south San Francisco Bay sloughs and the south San Francisco Bay result in an increase in the food available in the sloughs to their predators either through changes in biomass, availability, or palatability of benthic organisms.

True-bivalve biomass is high in summer and fall except in Artesian Slough, where bivalves did not establish a signifcant presence. The species present also contribute to the prey value of the community to predators. Potamocorbula amurensis is a shallow-burrowing bivalve. Given its position in the substrate, it is easy prey for bottom-feeding predators. Macoma is a deposit feeder and can burrow deeper into the substrate than Potamocorbula amurensis, making it harder to be preyed upon. There were also more amphipods in the sloughs in March, and that might have been important for predators.

\section{References Cited}

Cloern, J.E., 2001, Our evolving conceptual model of the coastal eutrophication problem: Marine Ecology Progress Series, v. 210, p. 223-253.

Cloern, J.E., Jassby, A.D., Thompson, J.K., Hieb, K., 2007, A cold phase of the east Pacific triggers new phytoplankton blooms in San Francisco Bay: Proceedings of the National Academy of Sciences, v. 104, no. 47, p. 18561-18565.

Hornberger, M., Luoma, S., Cain, D., Parchaso, F., Brown, C., Bouse, R., Wellise, C., and Thompson, J., 2000, Linkage of bioaccumulation and biological effects to changes in pollutant loads in South San Francisco Bay: Environmental Science and Technology, v. 34, p. 2401-2409.

Jassby, A.D., Cloern, J.E., Powell, T.M., 1993, Organic carbon sources and sinks in San Francisco Bay; variability induced by river flow: Marine Ecology Progress Series, v. 95, p. 39-54.

Lee, H.L., III, Thompson, B., and Lowe, S., 2003, Estuarine and scalar patterns of invasion in the softbottom benthic communities of the San Francisco Estuary: Biological Invasions, v. 5, p. 85-102.

Lucas, L.V., Koseff, J.R., Monismith, S.G., Thompson, J.K., 2009, Shallow water processes govern system-wide bloom dynamics; A modeling study: Journal of Marine Systems, v. 75, nos. 1-2, p. 7086.

Nichols, F.H., and Thompson, J.K., 1985a, Persistence of an introduced mudflat community in South San Francisco Bay, California: Marine Ecology Progress Series, v. 24, p. 83-97.

Nichols, F.H., and Thompson, J.K., 1985b, Time scales of change in the San Francisco Bay benthos: Hydrobiologia, v. 129, p. 121-138.

Poulton, V.K., Lovvorn, J.R., Takekawa, J.Y., 2002, Clam density and scaup feeding behavior in San Pablo Bay, California: Condor, v. 104, p. 518-527.

Poulton, V.K., Lovvorn, J.R., Takekawa, J.Y., 2004, Spatial and overwinter changes in clam populations of San Pablo Bay, a semiarid estuary with highly variable freshwater inflow: Estuarine Coastal and Shelf Science, v. 59, no. 3, p. 459-473. 
Richman, S.E., and Lovvorn, J.R., 2004, Relative foraging value to Lesser Scaup ducks of native and exotic clams from San Francisco Bay: Ecological Applications, v. 14, no. 4, p. 1217-1232.

Thompson, J.K., Koseff, J.R., Monismith, S.G., Lucas, L.V., 2008, Shallow water processes govern system-wide phytoplankton bloom dynamics; A field study: Journal of Marine Systems, v. 74, nos. 12, p. 153-166. 


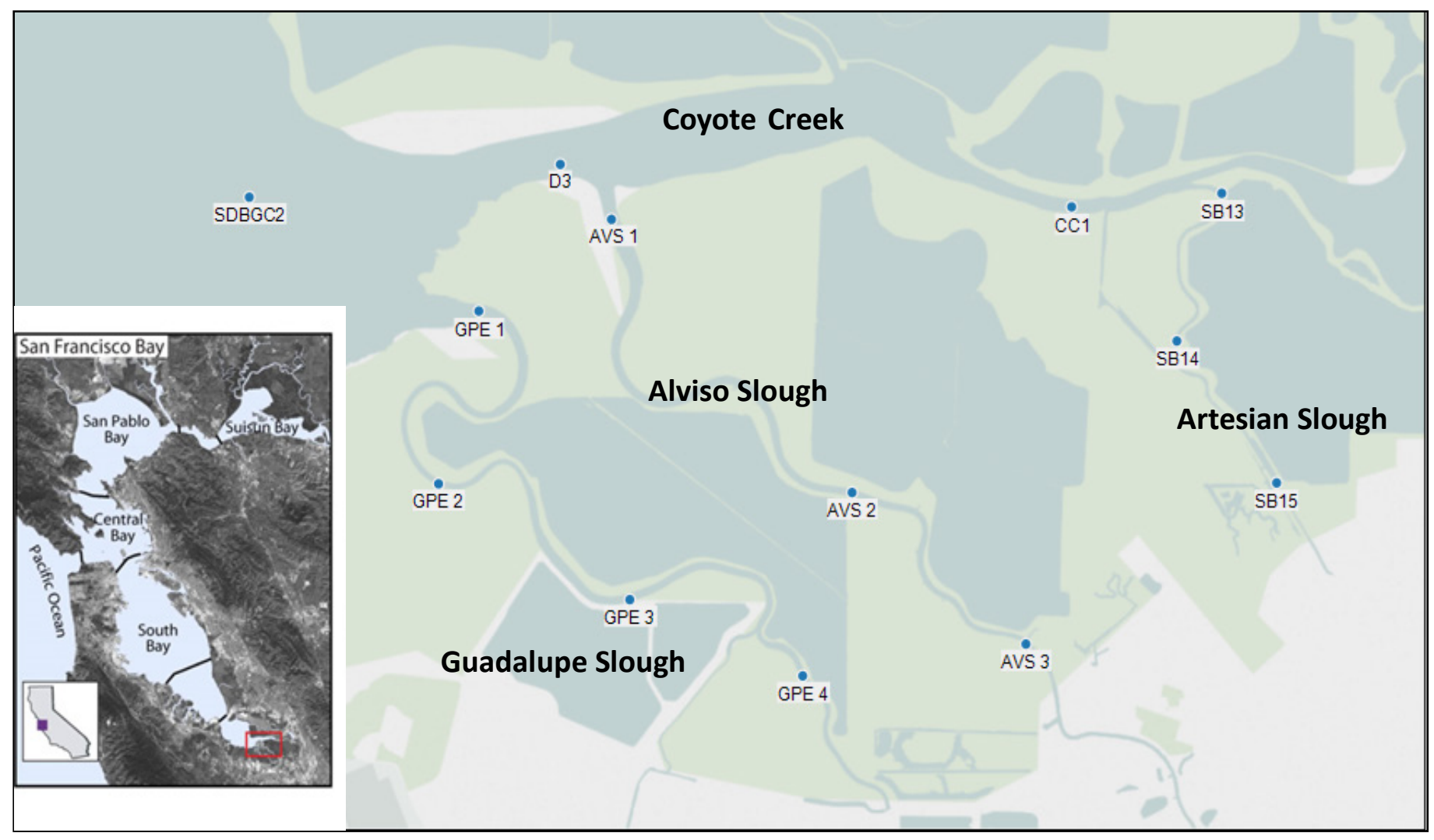

Figure 1. Map showing benthic sampling station locations in south San Francisco Bay and sampling area in relaton to San Francisco Bay proper. 


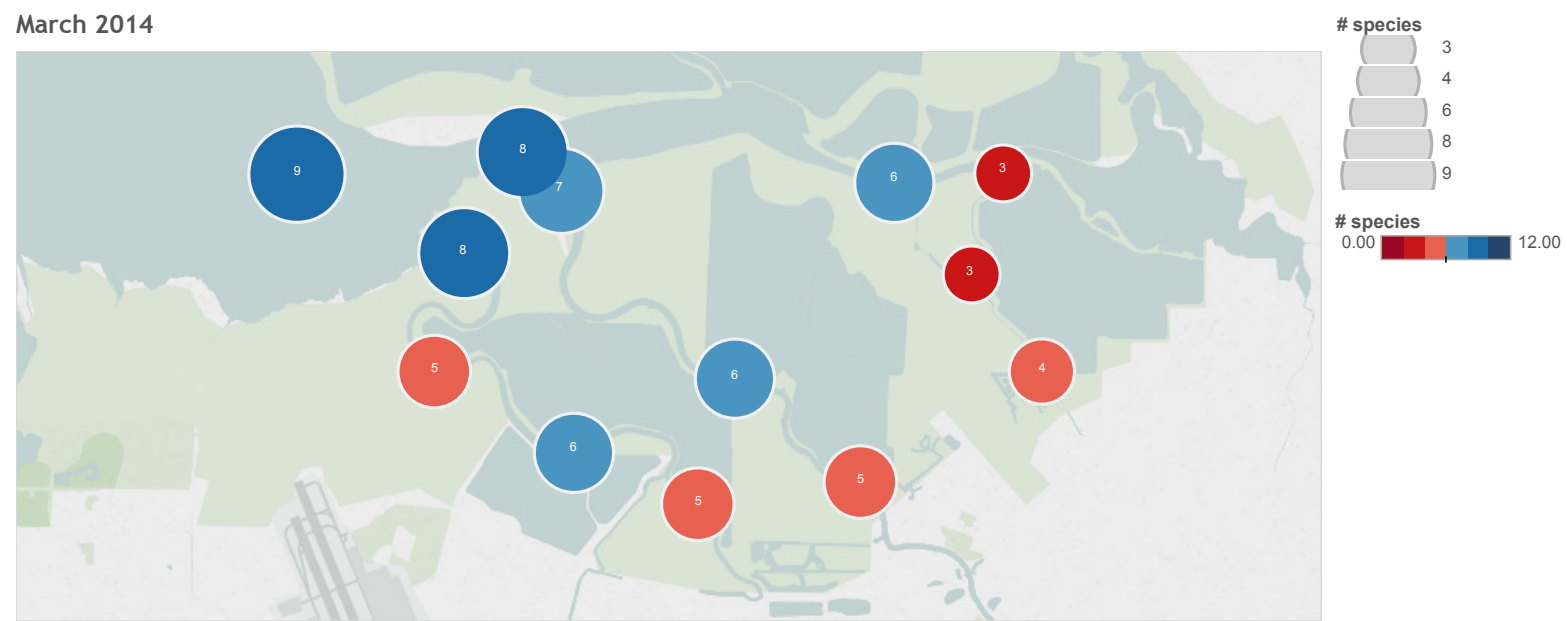

June 2014
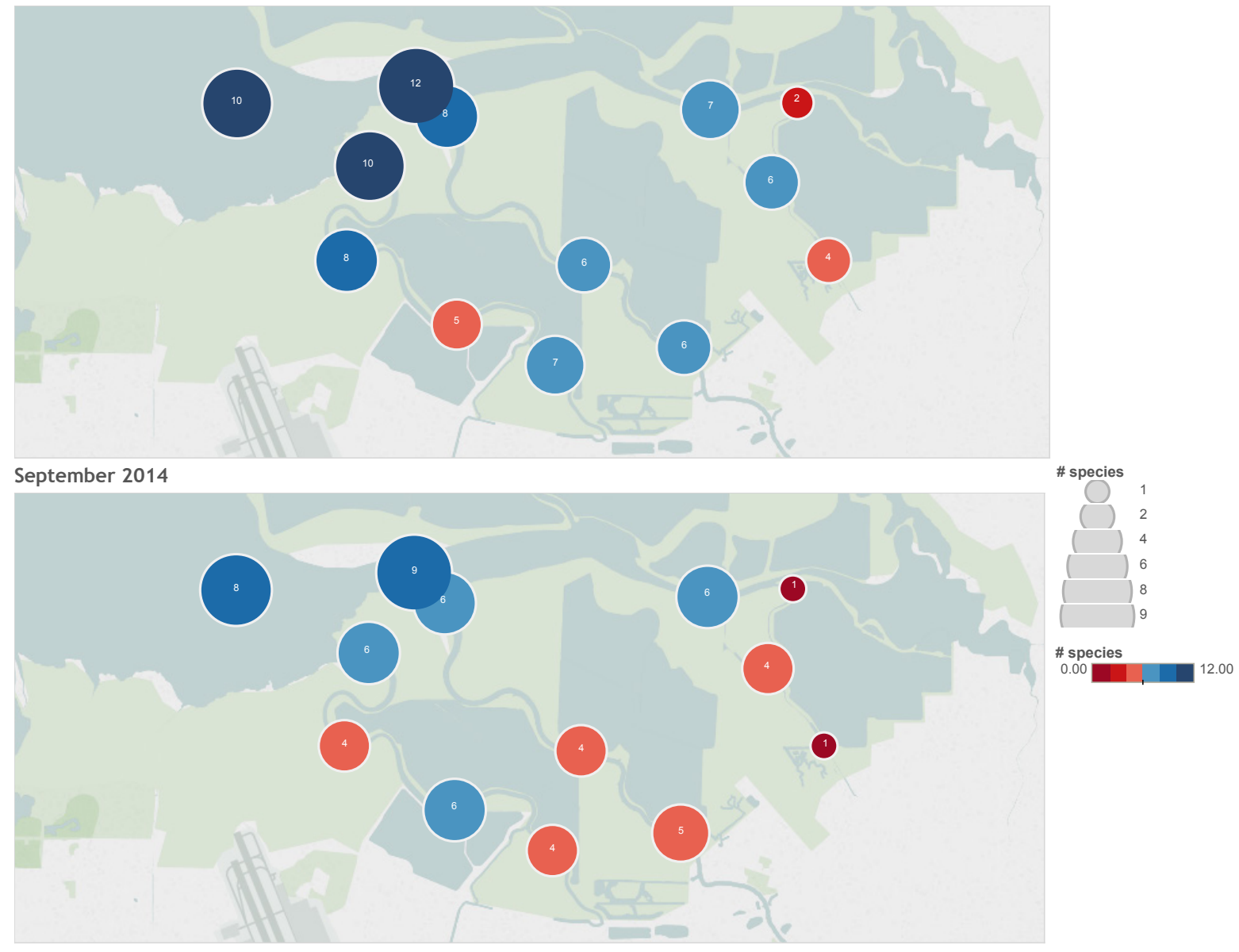

Figure 2. Maps showing the number of species at each station in south San Francisco Bay and its adjacent sloughs for March, June, and September 2014. 


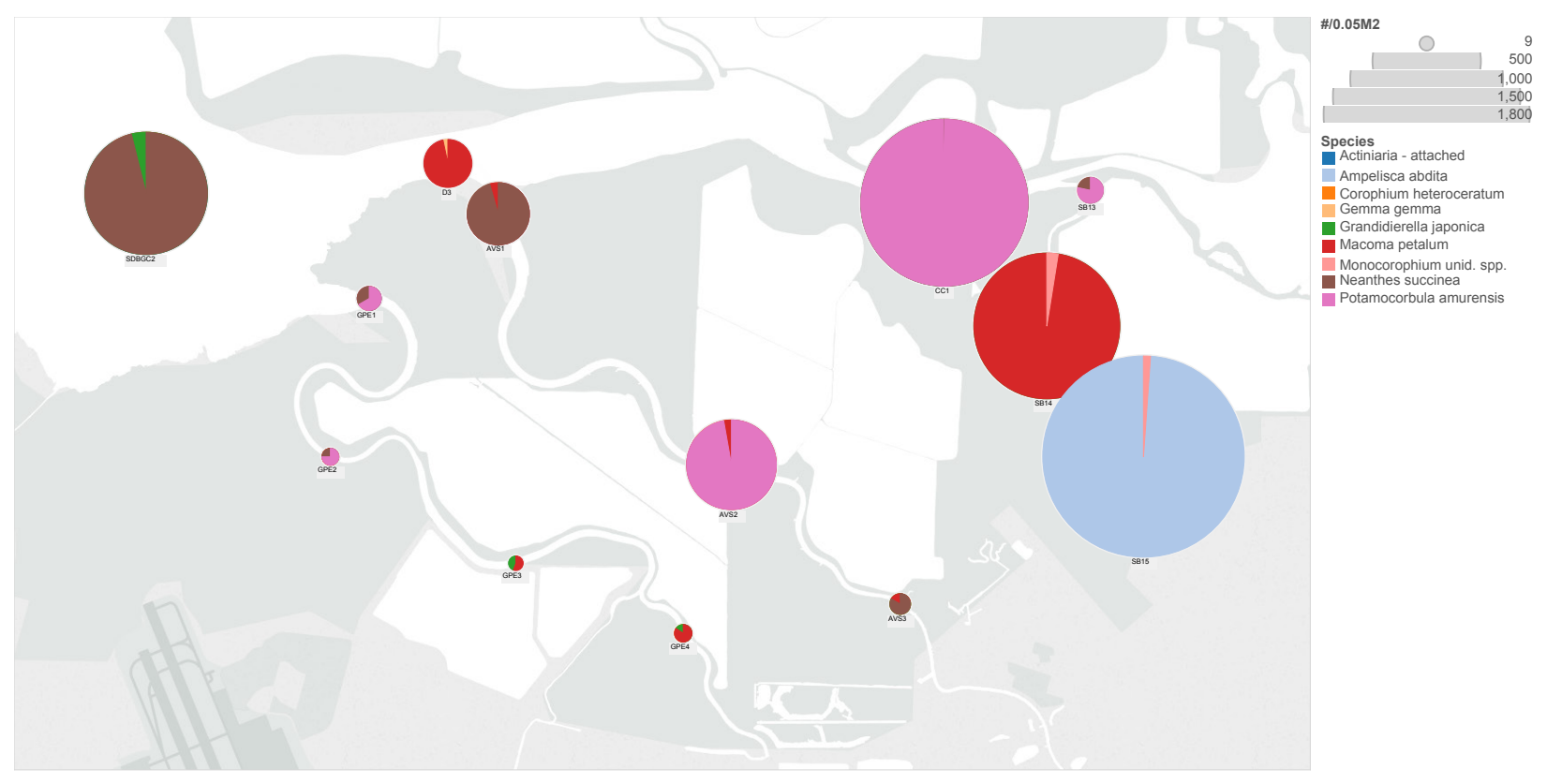

Figure 3. Map showing abundance of all species at all stations in March 2014 (for species where the abundance is greater than 2 per 0.05 square meter).

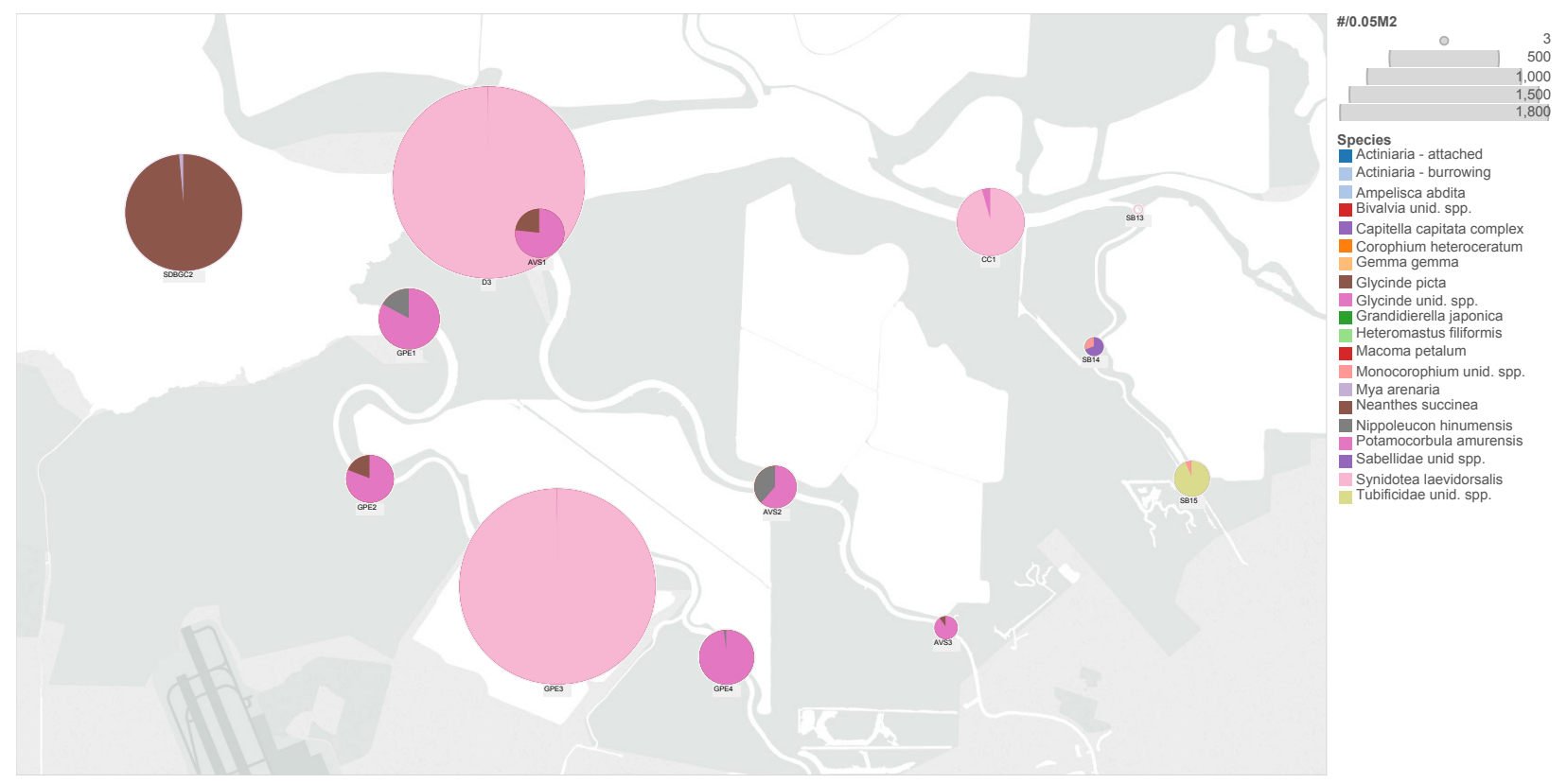

Figure 4. Map showing abundance of all species at all stations in June 2014 for species where the abundance is greater than 2 per 0.05 square meter). 


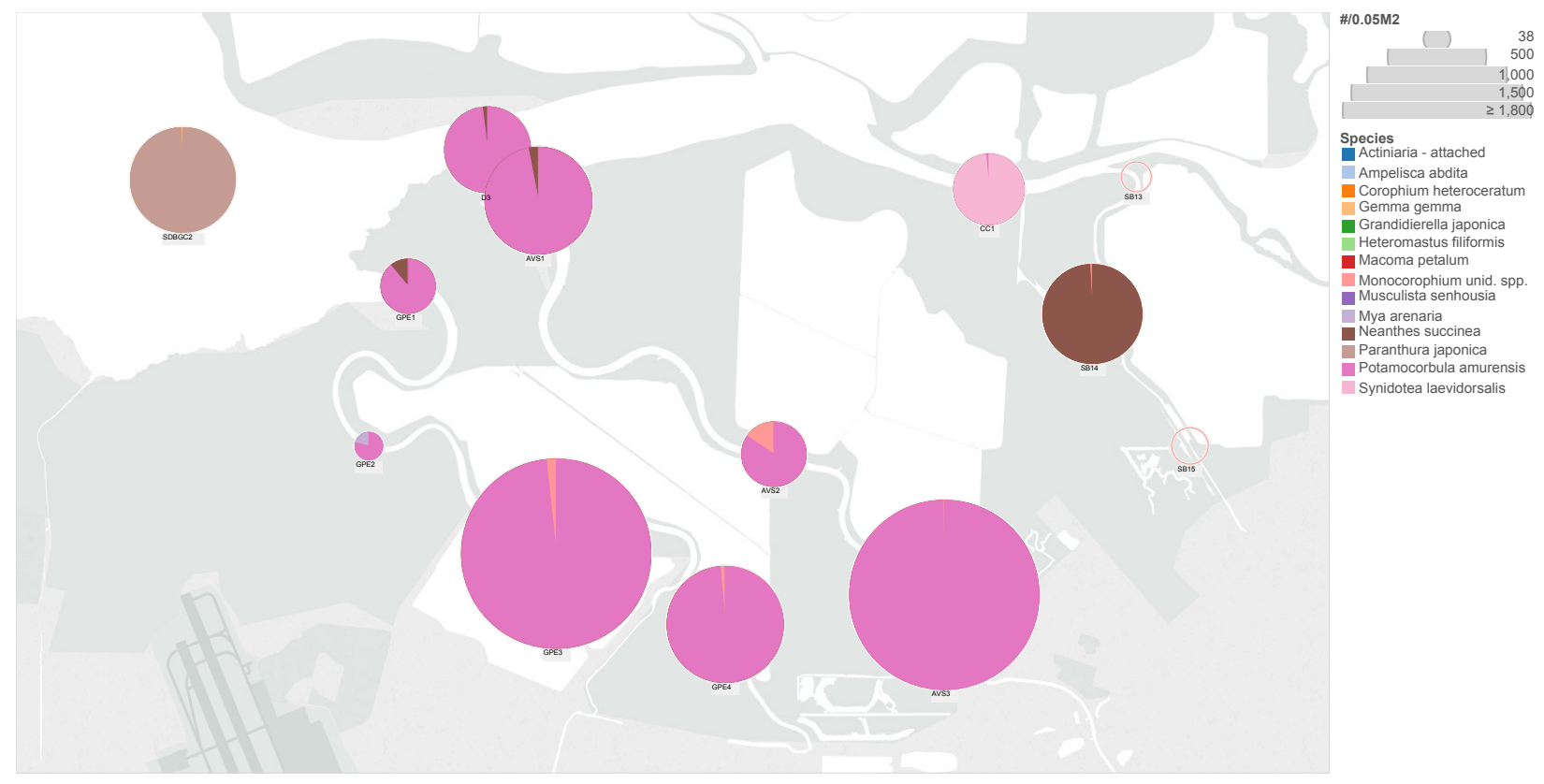

Figure 5. Map showing abundance of all species at all stations in September 2014 (for species where the abundance is greater than 2 per 0.05 square meter). 


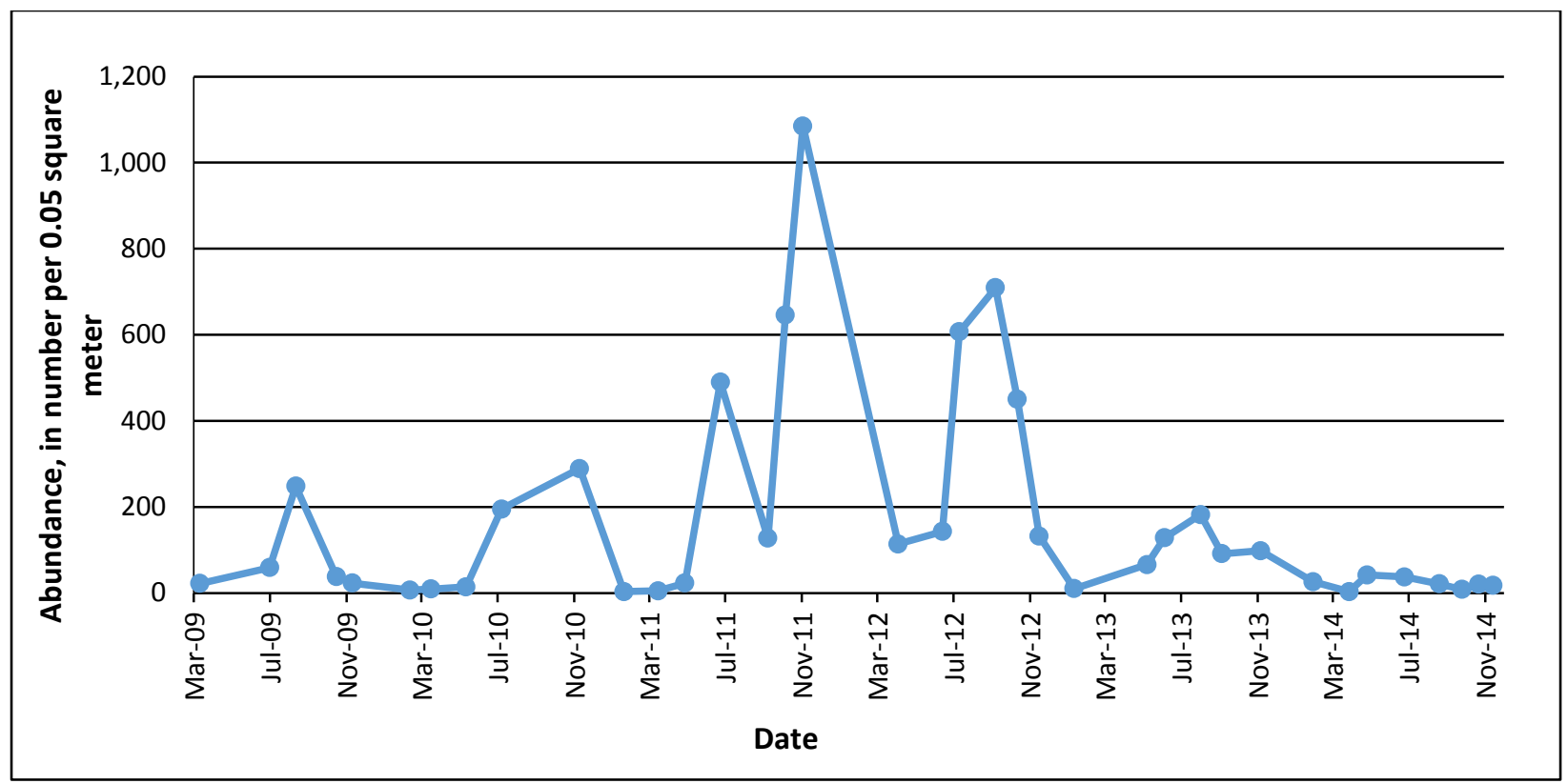

Figure 6. Time series graph showing abundance of class Bivalvia at station D3 in Coyote Creek, 2009-2014.

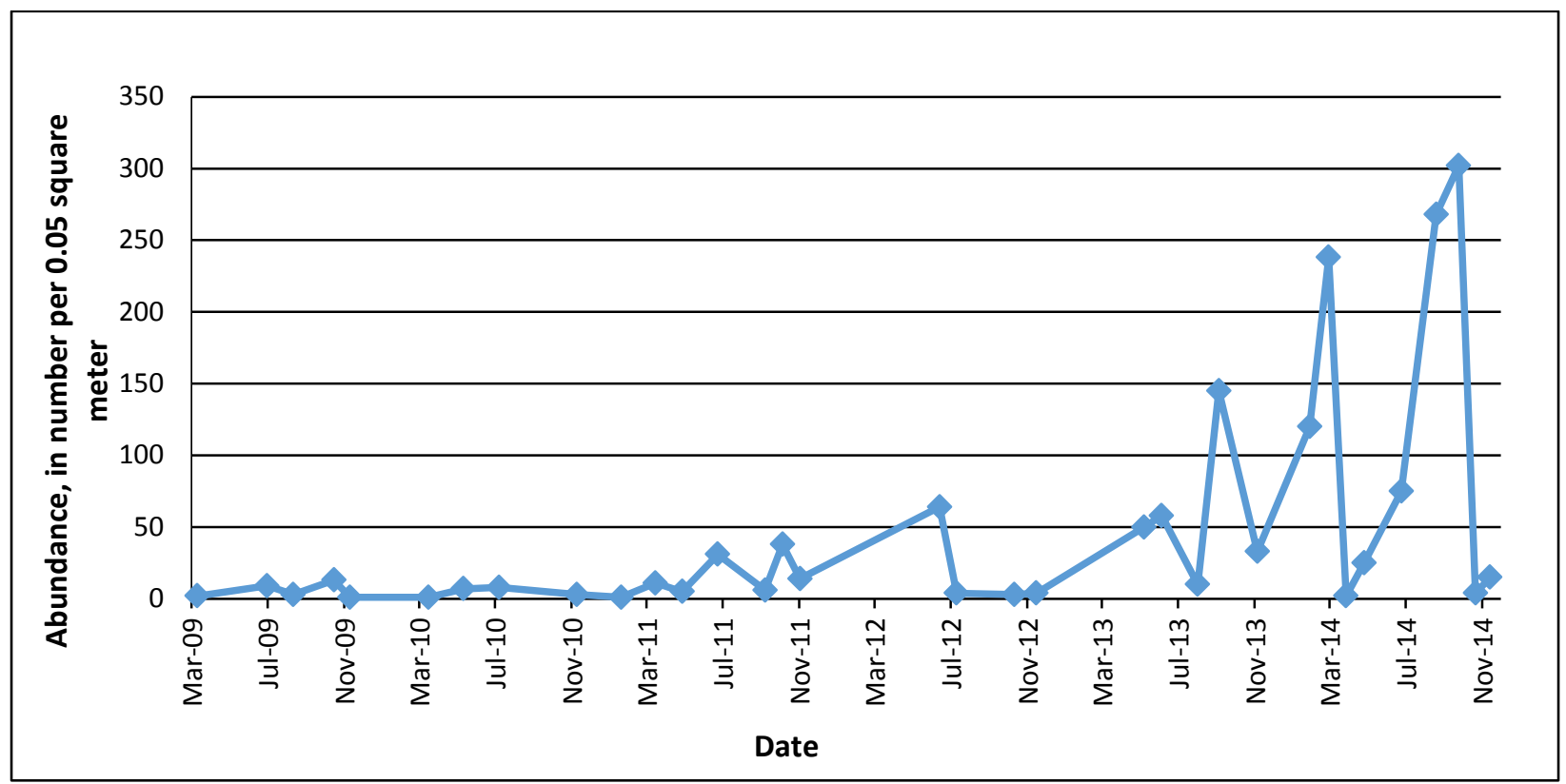

Figure 7. Time series graph showing abundance of Order Amphipoda at station D3 in Coyote Creek, 20092014. 


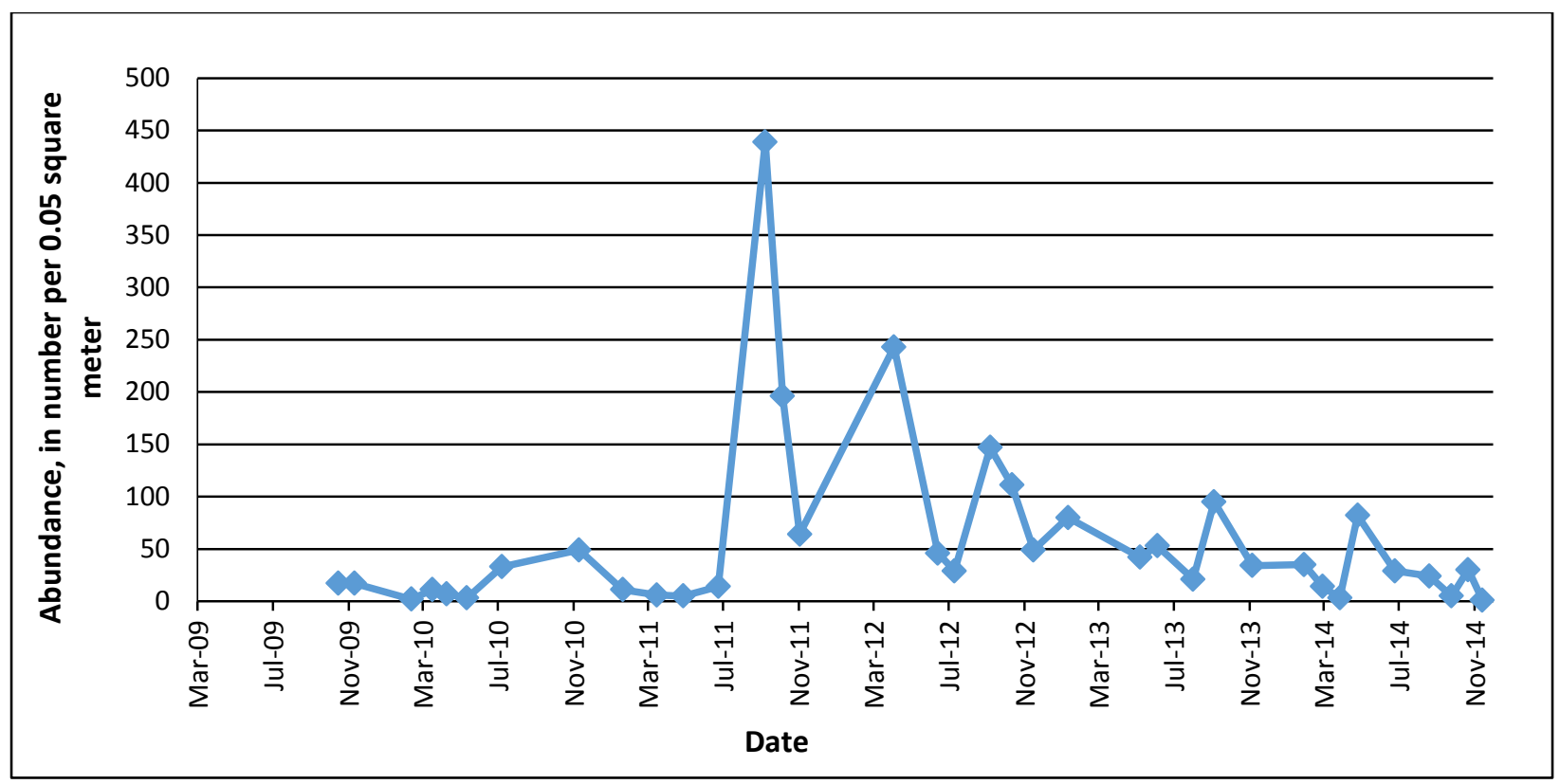

Figure 8. Time series graph showing abundance of class Bivalvia at station CC1 in Coyote Creek, 2009-2014.

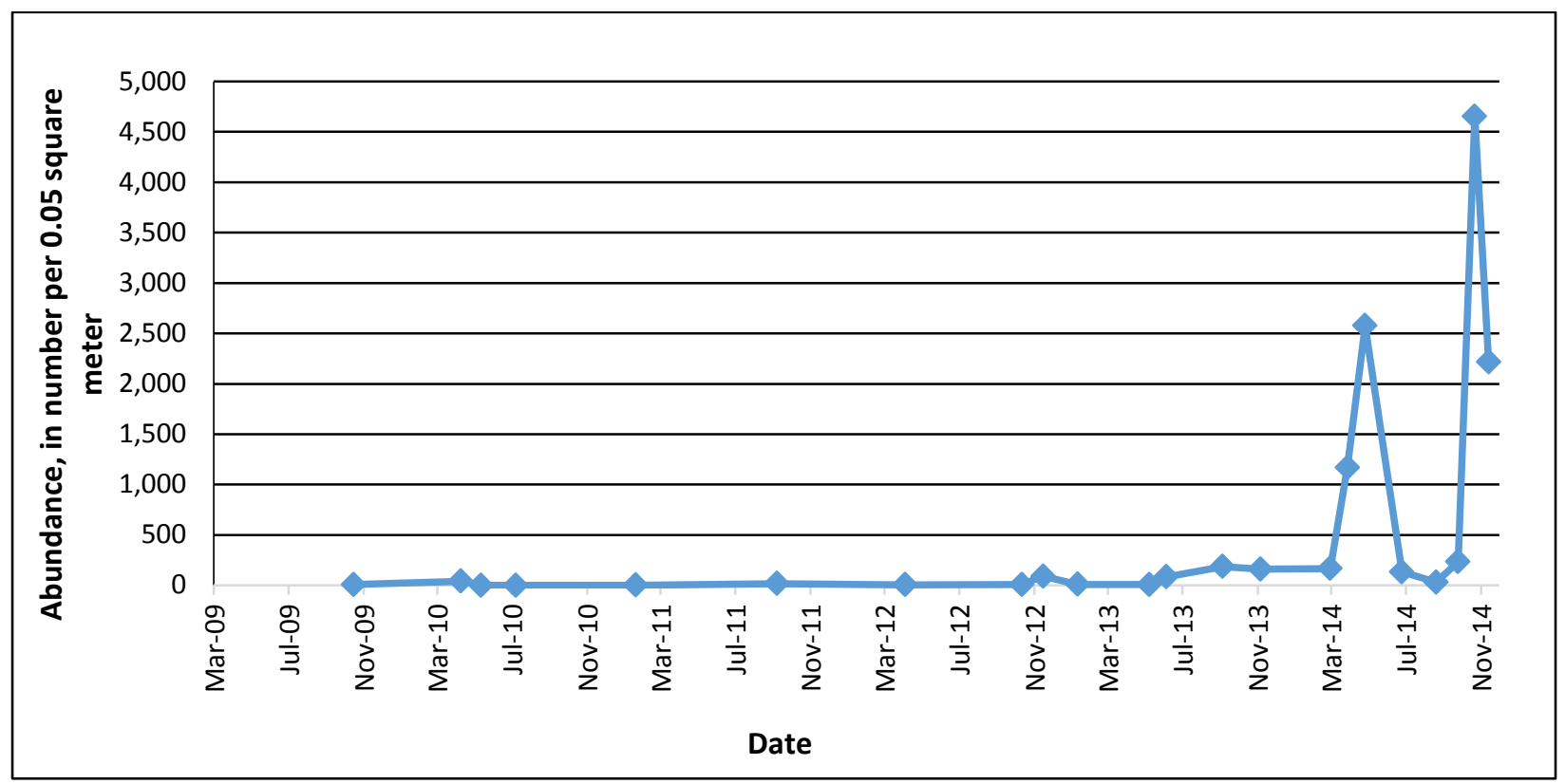

Figure 9. Time series graph showing abundance of Order Amphipoda at station CC1 in Coyote Creek, 20092014 


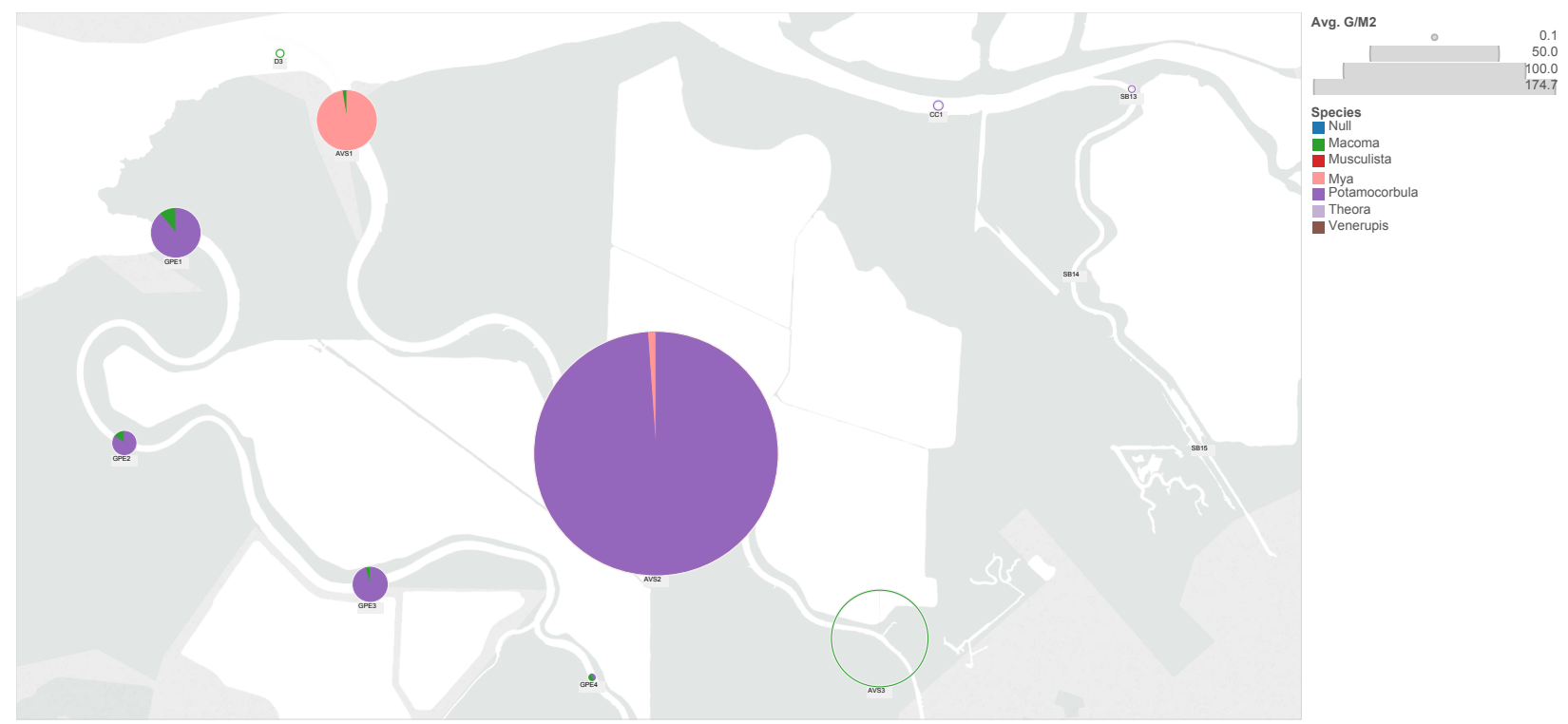

Figure 10. Map showing bivalve biomass at sampling stations in Guadalupe Slough, Alviso Slough, Artisean Slough, and Coyote Creek in March 2014. 


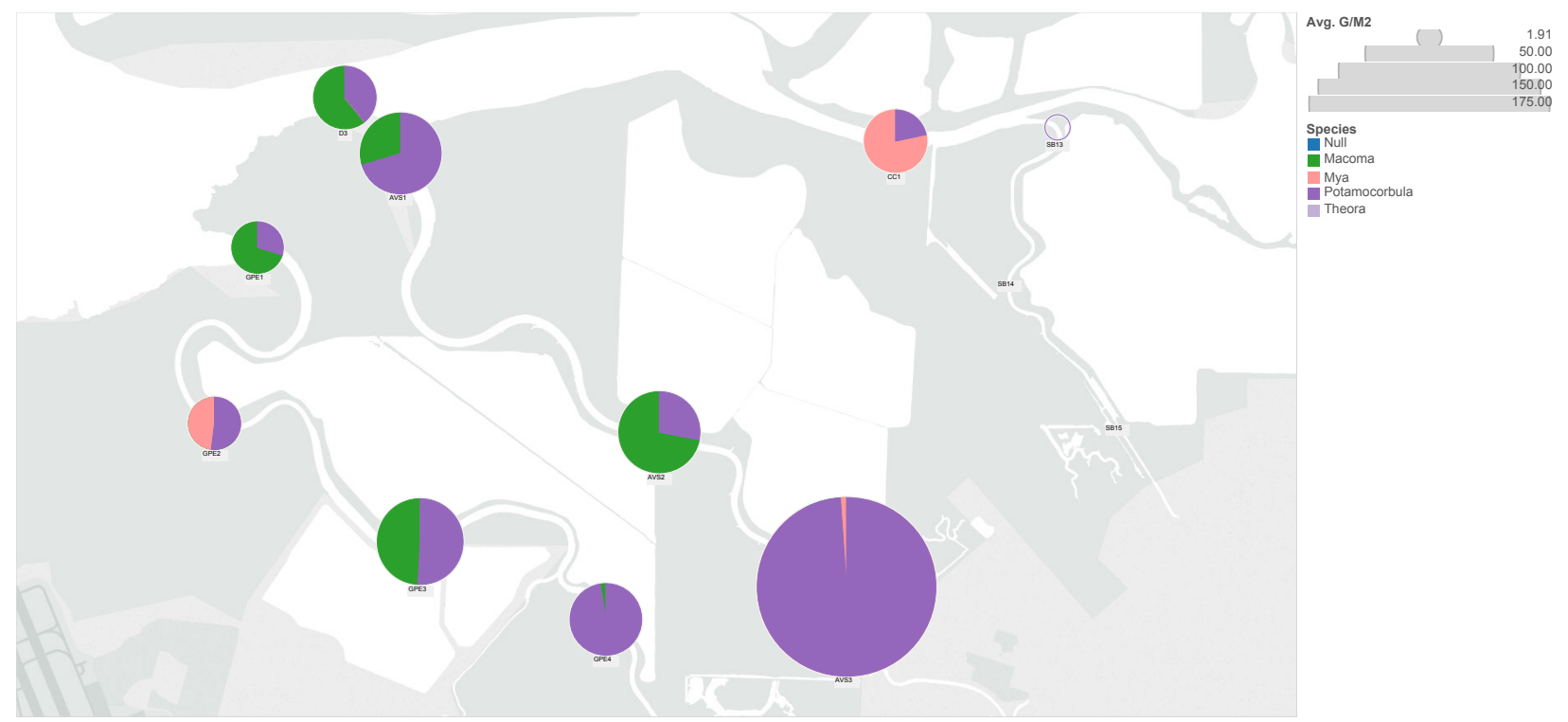

Figure 11. Map showing bivalve biomass at sampling stations in Guadalupe Slough, Alviso Slough, Artisean Slough, and Coyote Creek in June 2014.

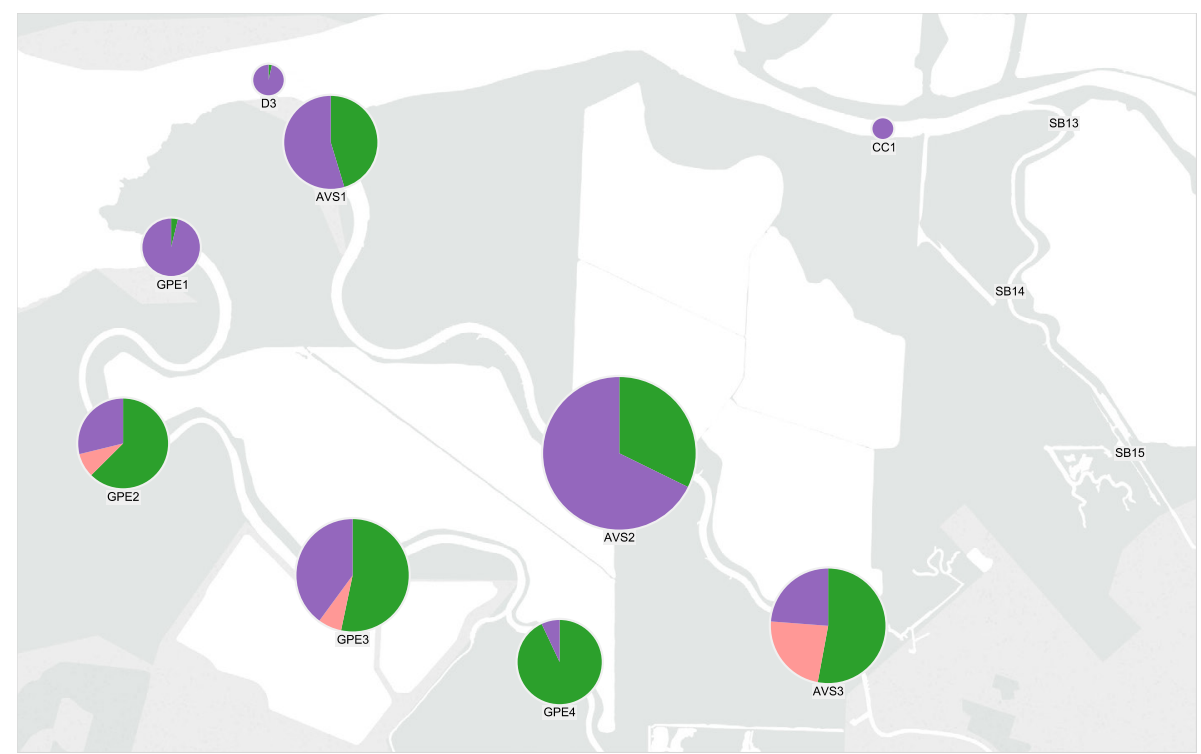

Figure 12. Map showing bivalve biomass at sampling stations in Guadalupe Slough, Alviso Slough, Artisean Slough, and Coyote Creek in September 2014. 


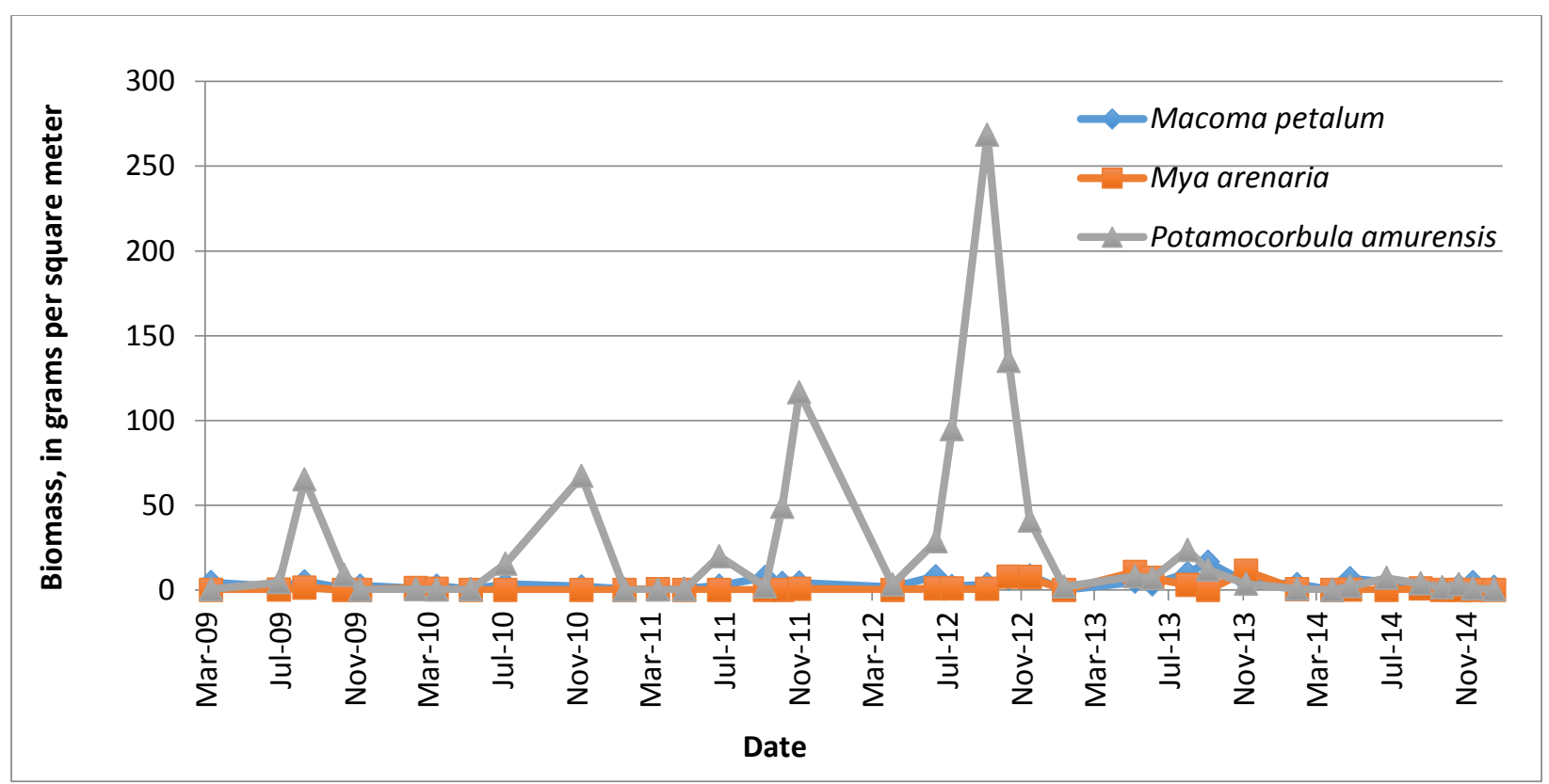

Figure 13. Time series graph showing bivalve biomass at station D3 in Coyote Creek, 2009-2014.

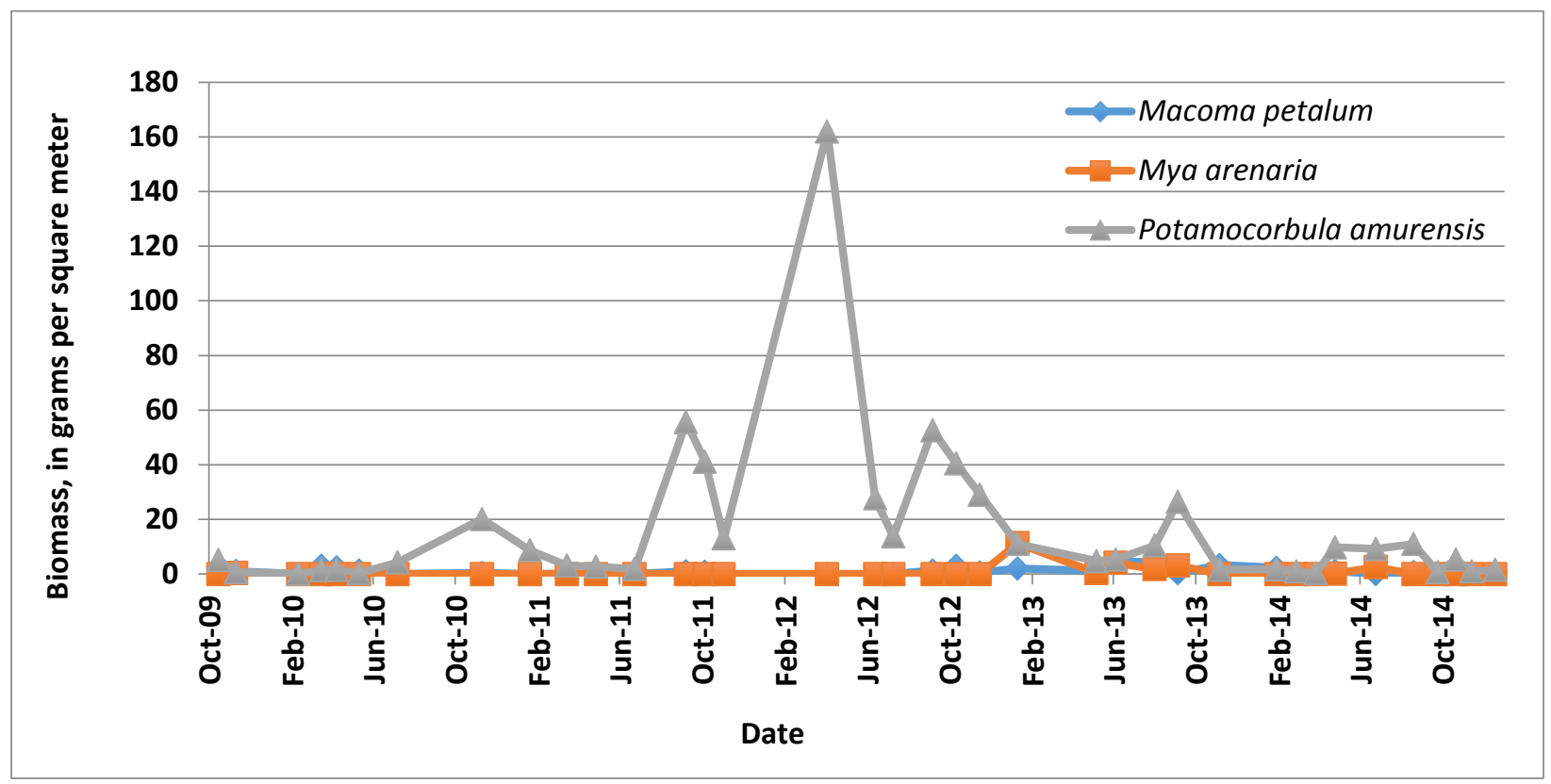

Figure 14. Time series graph showing bivalve biomass at station CC1 in Coyote Creek, 2009-2014. 
Table 1. Benthic sampling station coordinates (latitude-longitude).

\begin{tabular}{lll}
\hline \multicolumn{1}{c}{ Station } & \multicolumn{2}{c}{ Coordinates } \\
\hline AVS 1 & $\mathrm{N} 37.4591$ & $\mathrm{~W} 122.0209$ \\
AVS 2 & $\mathrm{N} 37.4399$ & $\mathrm{~W} 121.9984$ \\
AVS 3 & $\mathrm{N} 37.4193$ & $\mathrm{~W} 121.9821$ \\
GPE 1 & $\mathrm{N} 37.4526$ & $\mathrm{~W} 122.0334$ \\
GPE 2 & $\mathrm{N} 37.4405$ & $\mathrm{~W} 122.0371$ \\
GPE 3 & $\mathrm{N} 37.4323$ & $\mathrm{~W} 122.0192$ \\
GPE 4 & $\mathrm{N} 37.4269$ & $\mathrm{~W} 122.0031$ \\
SB13 & $\mathrm{N} 37.4609$ & $\mathrm{~W} 121.9637$ \\
SB14 & $\mathrm{N} 37.4505$ & $\mathrm{~W} 121.9679$ \\
SB15 & $\mathrm{N} 37.4405$ & $\mathrm{~W} 121.9586$ \\
D3 & $\mathrm{N} 37.4630$ & $\mathrm{~W} 122.0258$ \\
CC1 & $\mathrm{N} 37.4600$ & $\mathrm{~W} 121.9778$ \\
SDBGC2 & $\mathrm{N} 37.4607$ & $\mathrm{~W} 122.0549$ \\
\hline
\end{tabular}




\section{Appendixes}

Data are shown in the following units:

Biomas - grams ash-free-dry-mass/square meters $\left(\mathrm{g} / \mathrm{m}^{2}\right)$

Species and abundance-(number $/ 0.05 / \mathrm{m}^{2}$ )

Abbreviations:

sp.-Denotes a single species identified to the genus level spp.-Denotes many species of the same genera

(1), (2), SF1 - Denotes separate species of the same genera 
Appendix 1. AVS1 Species List and Abundance

\begin{tabular}{|c|c|c|c|c|c|c|c|}
\hline TAXON & 27-Mar-14 & 24-Jun-14 & 24-Sep-14 & TAXON & 27-Mar-14 & 24-Jun-14 & 24-Sep-14 \\
\hline $\begin{array}{l}\text { PHYLUM ANNELIDA } \\
\text { Class Oligochaeta } \\
\text { Naididae unid. spp. }\end{array}$ & 0.0 & 0.0 & 0.0 & $\begin{array}{l}\text { PHYLUM BRYOZOA } \\
\text { Class Gymnolaemata } \\
\text { Cheilostomatida unid. Spp. }\end{array}$ & 0.0 & 0.0 & 0.0 \\
\hline Oligochaeta unid. spp. & 0.0 & 0.0 & 0.0 & PHYLUM CHORDATA & & & \\
\hline Tubificidae unid. spp. & 0.0 & 0.0 & 0.0 & Class Ascidiacea & & & \\
\hline Class Polychaeta & & & & Molgula mahattensis & 0.0 & 0.0 & 0.0 \\
\hline Capitella capitata complex & 0.0 & 0.0 & 0.0 & PHYLUM CNIDARIA & & & \\
\hline Cirratulidae unid. spp. & 0.0 & 0.0 & 0.0 & Class Anthozoa & & & \\
\hline Eteone fauchaldi & 0.0 & 0.0 & 0.0 & Actiniaria - attached & 0.0 & 2.0 & 6.0 \\
\hline Eteone lighti & 0.0 & 0.0 & 0.0 & Actiniaria - burrowing & 0.0 & 0.0 & 0.0 \\
\hline Eteone unid. spp. & 0.0 & 0.0 & 0.0 & Actiniaria unid. spp. & 0.0 & 0.0 & 0.0 \\
\hline Euchone limnicola & 0.0 & 0.0 & 0.0 & Class Hydrozoa & & & \\
\hline Euchone unid. spp. & 0.0 & 0.0 & 0.0 & Hydrozoa unid spp. & 0.0 & 0.0 & 0.0 \\
\hline Exogone lourei & 0.0 & 0.0 & 0.0 & PHYLUM MOLLUSCA & & & \\
\hline Glycera unid. spp. & 0.0 & 0.0 & 0.0 & Class Bivalvia & & & \\
\hline Glycinde armigera & 0.0 & 0.0 & 0.0 & Bivalvia unid. spp. & 0.0 & 0.0 & 0.0 \\
\hline Glycinde picta & 0.0 & 0.0 & 0.0 & Gemma gemma & 118.0 & 27.0 & 0.0 \\
\hline Glycinde unid. sp. SF1 & 0.0 & 0.0 & 0.0 & Macoma petalum & 33.0 & 35.0 & 9.0 \\
\hline Glycinde unid. spp. & 0.0 & 0.0 & 0.0 & Musculista senhousia & 0.0 & 0.0 & 0.0 \\
\hline Harmothoe imbricata & 0.0 & 0.0 & 0.0 & Mya arenaria & 16.0 & 1.0 & 0.0 \\
\hline Heteromastus filiformis & 2.0 & 1.0 & 0.0 & Potamocorbula amurensis & $\begin{array}{c}10.0 \\
0.0\end{array}$ & 22.0 & 16.0 \\
\hline Leitoscoloplos pugettensis & 0.0 & 0.0 & 0.0 & Theora lubrica & 0.0 & $\begin{array}{c}22.0 \\
0.0\end{array}$ & $\begin{array}{c}10.0 \\
0.0\end{array}$ \\
\hline Maldanidae unid. spp. & 0.0 & 0.0 & 0.0 & Venerupis philippinarum & 0.0 & 0.0 & 0.0 \\
\hline Marphysa sanguinea & 0.0 & 0.0 & 0.0 & Class Gastropoda & & & \\
\hline Neanthes succinea & 7.0 & 5.0 & 8.0 & Crepidula unid. Spp. & 0.0 & 0.0 & 0.0 \\
\hline Polychaeta unid. spp. (1) & 0.0 & 0.0 & 0.0 & Gastropoda unid. sp. B & 0.0 & 0.0 & 0.0 \\
\hline Polychaeta unid. spp. (2) & 1 vial & 0.0 & 0.0 & $\begin{array}{l}\text { Gastropoda unia. sp. B } \\
\text { Ilyanassa obsoleta }\end{array}$ & 0.0 & 0.0 & 0.0 \\
\hline Polycirrus spp. & 0.0 & 0.0 & 0.0 & $\begin{array}{l}\text { lyyanassa obsoleta } \\
\text { Philine unid. spp. }\end{array}$ & $\begin{array}{l}0.0 \\
0.0\end{array}$ & 0.0 & 0.0 \\
\hline Polydora cornuta & 0.0 & 0.0 & 0.0 & Urosalpinx cinerea & 0.0 & 0.0 & 0.0 \\
\hline Polydora unid. spp. & 0.0 & 0.0 & 0.0 & PHYLUM NEMATODA & & & \\
\hline Pseudopolydora kempi & 0.0 & 0.0 & 0.0 & Nematoda unid. spp. & 0.0 & 0.0 & 0.0 \\
\hline Pseudopolydora paucibranchiata & 0.0 & 0.0 & 0.0 & & & & \\
\hline Sabaco elongatus & 0.0 & 0.0 & 0.0 & PHYLUM PLATYHELIMINTHES & & & \\
\hline Sabellidae unid. spp. & 0.0 & 0.0 & 0.0 & Class Turbellaria & & & \\
\hline Sphaerosyllis californiensis & 0.0 & 0.0 & 0.0 & Turbellaria unid. sp. A & 0.0 & 0.0 & 0.0 \\
\hline Sphaerosyllis erinaceus & 0.0 & 0.0 & 0.0 & Turbellaria unid. spp. & 0.0 & 0.0 & 0.0 \\
\hline Sphaerosyllis unid. sp. A & 0.0 & 0.0 & 0.0 & & & & \\
\hline Streblospio benedicti & 0.0 & 0.0 & 0.0 & & & & \\
\hline Spionidae unid. spp. & 0.0 & 0.0 & 0.0 & & & & \\
\hline PHYLUM ARTHROPODA & & & & & & & \\
\hline Class Copepoda & & & & & & & \\
\hline Calanoida unid. spp. & 0.0 & 0.0 & 0.0 & & & & \\
\hline Harpacticoida unid. spp. & 0.0 & 0.0 & 0.0 & & & & \\
\hline Class Malacostraca & & & & & & & \\
\hline Order Amphipoda & & & & & & & \\
\hline Ampelisca abdita & 5.0 & 4.0 & 507.0 & & & & \\
\hline Ampithoe unid. spp. & 0.0 & 0.0 & 0.0 & & & & \\
\hline Caprella californica & 0.0 & 0.0 & 0.0 & & & & \\
\hline Corophium heteroceratum & 0.0 & 0.0 & 26.0 & & & & \\
\hline Corophiidae unid. spp. & 0.0 & 0.0 & 0.0 & & & & \\
\hline Grandidierella japonica & 0.0 & 0.0 & 0.0 & & & & \\
\hline Monocorophium acherusicum & 0.0 & 0.0 & 0.0 & & & & \\
\hline Monocorophium insidiosum & 0.0 & 0.0 & 0.0 & & & & \\
\hline Monocorophium unid. spp. & 0.0 & 0.0 & 0.0 & & & & \\
\hline Oedicerotidae unid spp. & 0.0 & 0.0 & 0.0 & & & & \\
\hline Order Cumacea & & & & & & & \\
\hline Nippoleucon hinumensis & 11.0 & 0.0 & 0.0 & & & & \\
\hline Order Decapoda & & & & & & & \\
\hline Crangon franciscorum & 0.0 & 0.0 & 0.0 & & & & \\
\hline Hemigrapsus oregonensis & 0.0 & 0.0 & 0.0 & & & & \\
\hline Crab unid spp. & 0.0 & 0.0 & 0.0 & & & & \\
\hline Order Isopoda & & & & & & & \\
\hline Gnorisphaeroma oregonensis & 0.0 & 0.0 & 0.0 & & & & \\
\hline Paranthura japonica & 0.0 & 0.0 & 0.0 & & & & \\
\hline Synidotea laevidorsalis & 0.0 & 0.0 & 0.0 & & & & \\
\hline Synidotea unid. spp. & 0.0 & 0.0 & 0.0 & & & & \\
\hline Class Ostracoda & & & & & & & \\
\hline Eusarsiella zostericola & 0.0 & 0.0 & 0.0 & & & & \\
\hline Cyprideis unid. spp. & 0.0 & 0.0 & 0.0 & & & & \\
\hline Class Thecostraca & & & & & & & \\
\hline Balanomorpha unid. spp. & 0.0 & 0.0 & 0.0 & & & & \\
\hline Cirripedia unid. spp. & 0.0 & 0.0 & 0.0 & & & & \\
\hline
\end{tabular}


Appendix 2. AVS2 Species List and Abundance

\begin{tabular}{|c|c|c|c|c|c|c|c|}
\hline TAXON & 27-Mar-14 & 24-Jun-14 & 24-Sep-14 & TAXON & 27-Mar-14 & 24-Jun-14 & 24-Sep-14 \\
\hline $\begin{array}{l}\text { PHYLUM ANNELIDA } \\
\text { Class Oligochaeta } \\
\text { Naididae unid. spp. }\end{array}$ & 0.0 & 0.0 & 0.0 & $\begin{array}{l}\text { PHYLUM BRYOZOA } \\
\text { Class Gymnolaemata } \\
\text { Cheilostomatida unid. Spp. }\end{array}$ & 0.0 & 0.0 & 0.0 \\
\hline Oligochaeta unid. spp. & 0.0 & 0.0 & 0.0 & PHYLUM CHORDATA & & & \\
\hline Tubificidae unid. spp. & 5.0 & 0.0 & 0.0 & Class Ascidiacea & & & \\
\hline Class Polychaeta & & & & Molgula mahattensis & 0.0 & 0.0 & 0.0 \\
\hline Capitella capitata complex & 0.0 & 0.0 & 0.0 & PHYLUM CNIDARIA & & & \\
\hline Cirratulidae unid. spp. & 0.0 & 0.0 & 0.0 & Class Anthozoa & & & \\
\hline Eteone fauchaldi & 0.0 & 0.0 & 0.0 & Actiniaria - attached & 0.0 & 0.0 & 0.0 \\
\hline Eteone lighti & 1.0 & 1.0 & 0.0 & Actiniaria - burrowing & 0.0 & 0.0 & 0.0 \\
\hline Eteone unid. spp. & 0.0 & 0.0 & 0.0 & Actiniaria unid. spp. & 0.0 & 0.0 & 0.0 \\
\hline Euchone limnicola & 0.0 & 0.0 & 0.0 & Class Hydrozoa & & & \\
\hline Euchone unid. spp. & 0.0 & 0.0 & 0.0 & Hydrozoa unid spp. & 0.0 & 0.0 & 0.0 \\
\hline Exogone lourei & 0.0 & 0.0 & 0.0 & PHYLUM MOLLUSCA & & & \\
\hline Glycera unid. spp. & 0.0 & 0.0 & 0.0 & Class Bivalvia & & & \\
\hline Glycinde armigera & 0.0 & 0.0 & 0.0 & Bivalvia unid. spp. & 0.0 & 0.0 & 0.0 \\
\hline Glycinde picta & 0.0 & 0.0 & 0.0 & Gemma gemma & 0.0 & 0.0 & 0.0 \\
\hline Glycinde unid. sp. SF1 & 0.0 & 0.0 & 0.0 & Macoma petalum & 40.0 & 35.0 & 24.0 \\
\hline Glycinde unid. spp. & 0.0 & 0.0 & 0.0 & Musculista senhousia & 0.0 & 0.0 & 0.0 \\
\hline Harmothoe imbricata & 0.0 & 0.0 & 0.0 & Mya arenaria & 2.0 & 0.0 & 0.0 \\
\hline Heteromastus filiformis & 0.0 & 0.0 & 0.0 & Potamocorbula amurensis & 9.0 & 27.0 & 32.0 \\
\hline Leitoscoloplos pugettensis & 0.0 & 0.0 & 0.0 & Theora lubrica & 0.0 & 0.0 & $\begin{array}{c}32.0 \\
0.0\end{array}$ \\
\hline Maldanidae unid. spp. & 0.0 & 0.0 & 0.0 & Venerupis philippinarum & 0.0 & 0.0 & 0.0 \\
\hline Marphysa sanguinea & 0.0 & 0.0 & 0.0 & Class Gastropoda & & & \\
\hline Neanthes succinea & 0.0 & 0.0 & 0.0 & Crepidula unid. Spp. & 0.0 & 0.0 & 0.0 \\
\hline Polychaeta unid. spp. (1) & 0.0 & 0.0 & 0.0 & Gastropoda unid. sp. B & 0.0 & 0.0 & 0.0 \\
\hline Polychaeta unid. spp. (2) & 0.0 & 0.0 & 0.0 & $\begin{array}{l}\text { Gastropoda unia. sp. B } \\
\text { Ilyanassa obsoleta }\end{array}$ & 0.0 & 0.0 & 0.0 \\
\hline Polycirrus spp. & 0.0 & 0.0 & 0.0 & Philine unid. spp. & 0.0 & 0.0 & 0.0 \\
\hline Polydora cornuta & 0.0 & 0.0 & 0.0 & Urosalpinx cinerea & 0.0 & 0.0 & 0.0 \\
\hline Polydora unid. spp. & 0.0 & 0.0 & 0.0 & PHYLUM NEMATODA & & & \\
\hline Pseudopolydora kempi & 0.0 & 0.0 & 0.0 & Nematoda unid. spp. & 0.0 & 0.0 & 0.0 \\
\hline Pseudopolydora paucibranchiata & 0.0 & 0.0 & 0.0 & & & & \\
\hline Sabaco elongatus & 0.0 & 0.0 & 0.0 & PHYLUM PLATYHELIMINTHES & & & \\
\hline Sabellidae unid. spp. & 0.0 & 0.0 & 0.0 & Class Turbellaria & & & \\
\hline Sphaerosyllis californiensis & 0.0 & 0.0 & 0.0 & Turbellaria unid. sp. A & 0.0 & 0.0 & 0.0 \\
\hline Sphaerosyllis erinaceus & 0.0 & 0.0 & 0.0 & Turbellaria unid. spp. & 0.0 & 0.0 & 0.0 \\
\hline Sphaerosyllis unid. sp. A & 0.0 & 0.0 & 0.0 & & & & \\
\hline Streblospio benedicti & 0.0 & 0.0 & 0.0 & & & & \\
\hline Spionidae unid. spp. & 0.0 & 0.0 & 0.0 & & & & \\
\hline PHYLUM ARTHROPODA & & & & & & & \\
\hline Class Copepoda & & & & & & & \\
\hline Calanoida unid. spp. & 0.0 & 0.0 & 0.0 & & & & \\
\hline Harpacticoida unid. spp. & 0.0 & 0.0 & 0.0 & & & & \\
\hline Class Malacostraca & & & & & & & \\
\hline Order Amphipoda & & & & & & & \\
\hline Ampelisca abdita & 0.0 & 3.0 & 0.0 & & & & \\
\hline Ampithoe unid. spp. & 0.0 & 0.0 & 0.0 & & & & \\
\hline Caprella californica & 0.0 & 0.0 & 0.0 & & & & \\
\hline Corophium heteroceratum & 289.0 & 3.0 & 0.0 & & & & \\
\hline Corophiidae unid. spp. & 0.0 & 0.0 & 0.0 & & & & \\
\hline Grandidierella japonica & 0.0 & 0.0 & 6.0 & & & & \\
\hline Monocorophium acherusicum & 0.0 & 0.0 & 0.0 & & & & \\
\hline Monocorophium insidiosum & 0.0 & 0.0 & 0.0 & & & & \\
\hline Monocorophium unid. spp. & 0.0 & 0.0 & 146.0 & & & & \\
\hline Oedicerotidae unid spp. & 0.0 & 0.0 & 0.0 & & & & \\
\hline Order Cumacea & & & & & & & \\
\hline Nippoleucon hinumensis & 0.0 & 2.0 & 0.0 & & & & \\
\hline Order Decapoda & & & & & & & \\
\hline Crangon franciscorum & 0.0 & 0.0 & 0.0 & & & & \\
\hline Hemigrapsus oregonensis & 0.0 & 0.0 & 0.0 & & & & \\
\hline Crab unid spp. & 0.0 & 0.0 & 0.0 & & & & \\
\hline Order Isopoda & & & & & & & \\
\hline Gnorisphaeroma oregonensis & 0.0 & 0.0 & 0.0 & & & & \\
\hline Paranthura japonica & 0.0 & 0.0 & 0.0 & & & & \\
\hline Synidotea laevidorsalis & 0.0 & 0.0 & 0.0 & & & & \\
\hline Synidotea unid. spp. & 0.0 & 0.0 & 0.0 & & & & \\
\hline Class Ostracoda & & & & & & & \\
\hline Eusarsiella zostericola & 0.0 & 0.0 & 0.0 & & & & \\
\hline Cyprideis unid. spp. & 0.0 & 0.0 & 0.0 & & & & \\
\hline Class Thecostraca & & & & & & & \\
\hline Balanomorpha unid. spp. & 0.0 & 0.0 & 0.0 & & & & \\
\hline Cirripedia unid. spp. & 0.0 & 0.0 & 0.0 & & & & \\
\hline
\end{tabular}


Appendix 3. AVS3 Species List and Abundance

\begin{tabular}{|c|c|c|c|c|c|c|c|}
\hline TAXON & 27-Mar-14 & 24-Jun-14 & 24-Sep-14 & TAXON & 27-Mar-14 & 24-Jun-14 & 24-Sep-14 \\
\hline $\begin{array}{l}\text { PHYLUM ANNELIDA } \\
\text { Class Oligochaeta } \\
\text { Naididae unid. spp. }\end{array}$ & 0.0 & 0.0 & 0.0 & $\begin{array}{l}\text { PHYLUM BRYOZOA } \\
\text { Class Gymnolaemata } \\
\text { Cheilostomatida unid. Spp. }\end{array}$ & 0.0 & 0.0 & 0.0 \\
\hline Oligochaeta unid. spp. & 0.0 & 0.0 & 0.0 & PHYLUM CHORDATA & & & \\
\hline Tubificidae unid. spp. & 0.0 & 0.0 & 0.0 & Class Ascidiacea & & & \\
\hline Class Polychaeta & & & & Molgula mahattensis & 0.0 & 0.0 & 0.0 \\
\hline Capitella capitata complex & 0.0 & 0.0 & 0.0 & PHYLUM CNIDARIA & & & \\
\hline Cirratulidae unid. spp. & 0.0 & 0.0 & 0.0 & Class Anthozoa & & & \\
\hline Eteone fauchaldi & 0.0 & 0.0 & 0.0 & Actiniaria - attached & 0.0 & 0.0 & 0.0 \\
\hline Eteone lighti & 0.0 & 0.0 & 0.0 & Actiniaria - burrowing & 0.0 & 0.0 & 0.0 \\
\hline Eteone unid. spp. & 0.0 & 0.0 & 0.0 & Actiniaria unid. spp. & 0.0 & 0.0 & 0.0 \\
\hline Euchone limnicola & 0.0 & 0.0 & 0.0 & Class Hydrozoa & & & \\
\hline Euchone unid. spp. & 0.0 & 0.0 & 0.0 & Hydrozoa unid spp. & 0.0 & 0.0 & 0.0 \\
\hline Exogone lourei & 0.0 & 0.0 & 0.0 & PHYLUM MOLLUSCA & & & \\
\hline Glycera unid. spp. & 0.0 & 0.0 & 0.0 & Class Bivalvia & & & \\
\hline Glycinde armigera & 0.0 & 0.0 & 0.0 & Bivalvia unid. spp. & 0.0 & 0.0 & 0.0 \\
\hline Glycinde picta & 0.0 & 0.0 & 0.0 & Gemma gemma & 0.0 & 0.0 & 0.0 \\
\hline Glycinde unid. sp. SF1 & 0.0 & 0.0 & 0.0 & Macoma petalum & 11.0 & 12.0 & 18.0 \\
\hline Glycinde unid. spp. & 0.0 & 0.0 & 0.0 & Musculista senhousia & 0.0 & 0.0 & 0.0 \\
\hline Harmothoe imbricata & 0.0 & 0.0 & 0.0 & Mya arenaria & 0.0 & 2.0 & 1.0 \\
\hline Heteromastus filiformis & 0.0 & 1.0 & 0.0 & Potamocorbula amurensis & 0.0 & $\begin{array}{l}2.0 \\
2.0\end{array}$ & $\begin{array}{c}1.0 \\
10.0\end{array}$ \\
\hline Leitoscoloplos pugettensis & 0.0 & 0.0 & 0.0 & Theora lubrica & 0.0 & $\begin{array}{l}2.0 \\
0.0\end{array}$ & $\begin{array}{c}10.0 \\
0.0\end{array}$ \\
\hline Maldanidae unid. spp. & 0.0 & 0.0 & 0.0 & Venerupis philippinarum & 0.0 & 0.0 & 0.0 \\
\hline Marphysa sanguinea & 0.0 & 0.0 & 0.0 & Class Gastropoda & & & \\
\hline Neanthes succinea & 3.0 & 4.0 & 0.0 & Crepidula unid. Spp. & 0.0 & 0.0 & 0.0 \\
\hline Polychaeta unid. spp. (1) & 0.0 & 0.0 & 0.0 & Gastropoda unid. sp. B & 0.0 & 0.0 & 0.0 \\
\hline Polychaeta unid. spp. (2) & 0.0 & 0.0 & 0.0 & $\begin{array}{l}\text { Gastropoda unia. sp. B } \\
\text { Ilyanassa obsoleta }\end{array}$ & 0.0 & 0.0 & 0.0 \\
\hline Polycirrus spp. & 0.0 & 1.0 & 0.0 & Philine unid. spp. & 0.0 & 0.0 & 0.0 \\
\hline Polydora cornuta & 0.0 & 0.0 & 0.0 & Urosalpinx cinerea & 0.0 & 0.0 & 0.0 \\
\hline Polydora unid. spp. & 0.0 & 0.0 & 0.0 & PHYLUM NEMATODA & & & \\
\hline Pseudopolydora kempi & 0.0 & 0.0 & 0.0 & Nematoda unid. spp. & 0.0 & 0.0 & 0.0 \\
\hline Pseudopolydora paucibranchiata & 0.0 & 0.0 & 0.0 & & & & \\
\hline Sabaco elongatus & 0.0 & 0.0 & 0.0 & PHYLUM PLATYHELIMINTHES & & & \\
\hline Sabellidae unid. spp. & 0.0 & 0.0 & 0.0 & Class Turbellaria & & & \\
\hline Sphaerosyllis californiensis & 0.0 & 0.0 & 0.0 & Turbellaria unid. sp. A & 0.0 & 0.0 & 0.0 \\
\hline Sphaerosyllis erinaceus & 0.0 & 0.0 & 0.0 & Turbellaria unid. spp. & 0.0 & 0.0 & 0.0 \\
\hline Sphaerosyllis unid. sp. A & 0.0 & 0.0 & 0.0 & & & & \\
\hline Streblospio benedicti & 0.0 & 0.0 & 0.0 & & & & \\
\hline Spionidae unid. spp. & 0.0 & 0.0 & 0.0 & & & & \\
\hline PHYLUM ARTHROPODA & & & & & & & \\
\hline Class Copepoda & & & & & & & \\
\hline Calanoida unid. spp. & 0.0 & 0.0 & 0.0 & & & & \\
\hline Harpacticoida unid. spp. & 0.0 & 0.0 & 0.0 & & & & \\
\hline Class Malacostraca & & & & & & & \\
\hline Order Amphipoda & & & & & & & \\
\hline Ampelisca abdita & 0.0 & 0.0 & 57.0 & & & & \\
\hline Ampithoe unid. spp. & 0.0 & 0.0 & 0.0 & & & & \\
\hline Caprella californica & 0.0 & 0.0 & 0.0 & & & & \\
\hline Corophium heteroceratum & 2.0 & 1.0 & 0.0 & & & & \\
\hline Corophiidae unid. spp. & 0.0 & 0.0 & 0.0 & & & & \\
\hline Grandidierella japonica & 2.0 & 0.0 & 0.0 & & & & \\
\hline Monocorophium acherusicum & 0.0 & 0.0 & 0.0 & & & & \\
\hline Monocorophium insidiosum & 0.0 & 0.0 & 0.0 & & & & \\
\hline Monocorophium unid. spp. & 0.0 & 0.0 & 3573.0 & & & & \\
\hline Oedicerotidae unid spp. & 0.0 & 0.0 & 0.0 & & & & \\
\hline Order Cumacea & & & & & & & \\
\hline Nippoleucon hinumensis & 12.0 & 0.0 & 0.0 & & & & \\
\hline Order Decapoda & & & & & & & \\
\hline Crangon franciscorum & 0.0 & 0.0 & 0.0 & & & & \\
\hline Hemigrapsus oregonensis & 0.0 & 0.0 & 0.0 & & & & \\
\hline Crab unid spp. & 0.0 & 0.0 & 0.0 & & & & \\
\hline Order Isopoda & & & & & & & \\
\hline Gnorisphaeroma oregonensis & 0.0 & 0.0 & 0.0 & & & & \\
\hline Paranthura japonica & 0.0 & 0.0 & 0.0 & & & & \\
\hline Synidotea laevidorsalis & 0.0 & 0.0 & 0.0 & & & & \\
\hline Synidotea unid. spp. & 0.0 & 0.0 & 0.0 & & & & \\
\hline Class Ostracoda & & & & & & & \\
\hline Eusarsiella zostericola & 0.0 & 0.0 & 0.0 & & & & \\
\hline Cyprideis unid. spp. & 0.0 & 0.0 & 0.0 & & & & \\
\hline Class Thecostraca & & & & & & & \\
\hline Balanomorpha unid. spp. & 0.0 & 0.0 & 0.0 & & & & \\
\hline Cirripedia unid. spp. & 0.0 & 0.0 & 0.0 & & & & \\
\hline
\end{tabular}


Appendix 4. GPE1 Species List and Abundance

\begin{tabular}{|c|c|c|c|c|c|c|c|}
\hline TAXON & 27-Mar-14 & 24-Jun-14 & 24-Sep-14 & TAXON & 27-Mar-14 & 24-Jun-14 & 24-Sep-14 \\
\hline $\begin{array}{l}\text { PHYLUM ANNELIDA } \\
\text { Class Oligochaeta }\end{array}$ & & & & $\begin{array}{l}\text { PHYLUM BRYOZOA } \\
\text { Class Gymnolaemata }\end{array}$ & & & \\
\hline Naididae unid. spp. & 0.0 & 0.0 & 0.0 & Cheilostomatida unid. Spp. & 0.0 & 0.0 & 0.0 \\
\hline Oligochaeta unid. spp. & 0.0 & 0.0 & 0.0 & PHYLUM CHORDATA & & & \\
\hline Tubificidae unid. spp. & 0.0 & 0.0 & 0.0 & Class Ascidiacea & & & \\
\hline Class Polychaeta & & & & Molgula mahattensis & 0.0 & 0.0 & 0.0 \\
\hline Capitella capitata complex & 0.0 & 0.0 & 0.0 & PHYLUM CNIDARIA & & & \\
\hline Cirratulidae unid. spp. & 0.0 & 0.0 & 0.0 & Class Anthozoa & & & \\
\hline Eteone fauchaldi & 0.0 & 0.0 & 0.0 & Actiniaria - attached & 2.0 & 0.0 & 0.0 \\
\hline Eteone lighti & 1.0 & 0.0 & 0.0 & Actiniaria - burrowing & 0.0 & 0.0 & 0.0 \\
\hline Eteone unid. spp. & 0.0 & 0.0 & 0.0 & Actiniaria unid. spp. & 0.0 & 0.0 & 0.0 \\
\hline Euchone limnicola & 0.0 & 0.0 & 0.0 & Class Hydrozoa & & & \\
\hline Euchone unid. spp. & 0.0 & 0.0 & 0.0 & Hydrozoa unid spp. & 0.0 & 0.0 & 0.0 \\
\hline Exogone lourei & 0.0 & 0.0 & 0.0 & PHYLUM MOLLUSCA & & & \\
\hline Glycera unid. spp. & 0.0 & 0.0 & 0.0 & Class Bivalvia & & & \\
\hline Glycinde armigera & 0.0 & 0.0 & 0.0 & Bivalvia unid. spp. & 0.0 & 0.0 & 0.0 \\
\hline Glycinde picta & 0.0 & 0.0 & 0.0 & Gemma gemma & 0.0 & 8.0 & 0.0 \\
\hline Glycinde unid. sp. SF1 & 0.0 & 0.0 & 0.0 & Macoma petalum & 8.0 & 29.0 & 3.0 \\
\hline Glycinde unid. spp. & 0.0 & 0.0 & 0.0 & Musculista senhousia & 1.0 & 0.0 & 0.0 \\
\hline Harmothoe imbricata & 0.0 & 0.0 & 0.0 & Mya arenaria & 0.0 & 0.0 & 0.0 \\
\hline Heteromastus filiformis & 0.0 & 0.0 & 0.0 & Potamocorbula amurensis & 8.0 & 25.0 & 16.0 \\
\hline Maldanidae unid. spp. & 0.0 & 0.0 & 0.0 & Theora lubrica & 0.0 & 0.0 & 0.0 \\
\hline Leitoscoloplos pugettensis & 0.0 & 0.0 & 0.0 & Venerupis philippinarum & 0.0 & 0.0 & 0.0 \\
\hline Marphysa sanguinea & 0.0 & 0.0 & 0.0 & $\begin{array}{l}\text { Class Gastropoda } \\
\text { Clis philpin }\end{array}$ & & 0.0 & \\
\hline Neanthes succinea & 4.0 & 1.0 & 4.0 & $\begin{array}{l}\text { Crepsidula unid. Spp. } \\
\text { Creptropoda }\end{array}$ & 0.0 & 0.0 & 0.0 \\
\hline Polychaeta unid. spp. (1) & 0.0 & 0.0 & 0.0 & Gastropoda unid. sp. B & 0.0 & 0.0 & 0.0 \\
\hline Polychaeta unid. spp. (2) & 0.0 & 0.0 & 0.0 & llyanassa obsoleta & 1.0 & 0.0 & 0.0 \\
\hline Polycirrus spp. & 0.0 & 0.0 & 0.0 & Philine unid. spp. & 0.0 & 0.0 & 0.0 \\
\hline Polydora cornuta & 0.0 & 0.0 & 0.0 & Urosalpinx cinerea & 0.0 & 0.0 & 0.0 \\
\hline Polydora unid. spp. & 0.0 & 0.0 & 0.0 & PHYLUM NEMATODA & & & \\
\hline Pseudopolydora kempi & 0.0 & 0.0 & 0.0 & PHYYUM NEMAIODA & & & \\
\hline Pseudopolydora paucibranchiata & 0.0 & 0.0 & 0.0 & Nematoda unid. spp. & 0.0 & 0.0 & 0.0 \\
\hline Sabaco elongatus & 0.0 & 0.0 & 0.0 & PHYLUM PLATYHELMINTHES & & & \\
\hline Sabellidae unid. spp. & 0.0 & 0.0 & 0.0 & Class Turbellaria & & & \\
\hline Sphaerosyllis californiensis & 0.0 & 0.0 & 0.0 & Turbellaria unid. sp. A & 0.0 & 0.0 & 0.0 \\
\hline Sphaerosyllis erinaceus & 0.0 & 0.0 & 0.0 & Turbellaria unid. spp. & 0.0 & 0.0 & 0.0 \\
\hline Sphaerosyllis unid. sp. A & 0.0 & 0.0 & 0.0 & & & & \\
\hline Streblospio benedicti & 0.0 & 0.0 & 0.0 & & & & \\
\hline Spionidae unid. spp. & 0.0 & 0.0 & 0.0 & & & & \\
\hline \multicolumn{8}{|l|}{ PHYLUM ARTHROPODA } \\
\hline \multicolumn{8}{|l|}{ Class Copepoda } \\
\hline Calanoida unid. spp. & 0.0 & 0.0 & 0.0 & & & & \\
\hline Harpacticoida unid. spp. & 0.0 & 0.0 & 0.0 & & & & \\
\hline \multicolumn{8}{|l|}{ Class Malacostraca } \\
\hline \multicolumn{8}{|l|}{ Order Amphipoda } \\
\hline Ampelisca abdita & 0.0 & 21.0 & 86.0 & & & & \\
\hline Ampithoe unid. spp. & 0.0 & 0.0 & 0.0 & & & & \\
\hline Caprella californica & 0.0 & 0.0 & 0.0 & & & & \\
\hline Corophium heteroceratum & 0.0 & 17.0 & 0.0 & & & & \\
\hline Corophiidae unid. spp. & 0.0 & 0.0 & 0.0 & & & & \\
\hline Grandidierella japonica & 0.0 & 1.0 & 3.0 & & & & \\
\hline Monocorophium acherusicum & 0.0 & 0.0 & 0.0 & & & & \\
\hline Monocorophium insidiosum & 0.0 & 0.0 & 0.0 & & & & \\
\hline Monocorophium unid. spp. & 2.0 & 36.0 & 35.0 & & & & \\
\hline Oedicerotidae unid spp. & 0.0 & 0.0 & 0.0 & & & & \\
\hline \multicolumn{8}{|l|}{ Order Cumacea } \\
\hline Nippoleucon hinumensis & 0.0 & 11.0 & 0.0 & & & & \\
\hline \multicolumn{8}{|l|}{ Order Decapoda } \\
\hline Crangon franciscorum & 0.0 & 0.0 & 0.0 & & & & \\
\hline Hemigrapsus oregonensis & 0.0 & 0.0 & 0.0 & & & & \\
\hline Crab unid spp. & 0.0 & 0.0 & 0.0 & & & & \\
\hline \multicolumn{8}{|l|}{ Order Isopoda } \\
\hline Gnorisphaeroma oregonensis & 0.0 & 0.0 & 0.0 & & & & \\
\hline Paranthura japonica & 0.0 & 0.0 & 0.0 & & & & \\
\hline Synidotea laevidorsalis & 0.0 & 1.0 & 0.0 & & & & \\
\hline Synidotea unid. spp. & 0.0 & 0.0 & 0.0 & & & & \\
\hline \multicolumn{8}{|l|}{ Class Ostracoda } \\
\hline Eusarsiella zostericola & 0.0 & 0.0 & 0.0 & & & & \\
\hline Cyprideis unid. spp. & 0.0 & 0.0 & 0.0 & & & & \\
\hline \multicolumn{8}{|l|}{ Class Thecostraca } \\
\hline Balanomorpha unid. spp. & 0.0 & 0.0 & 0.0 & & & & \\
\hline Cirripedia unid. spp. & 0.0 & 0.0 & 0.0 & & & & \\
\hline
\end{tabular}


Appendix 5. GPE2 Species List And Abundance

\begin{tabular}{|c|c|c|c|c|c|c|c|}
\hline TAXON & 27-Mar-14 & 24-Jun-14 & 24-Sep-14 & TAXON & 27-Mar-14 & 24-Jun-14 & 24-Sep-14 \\
\hline $\begin{array}{l}\text { PHYLUM ANNELIDA } \\
\text { Class Oligochaeta } \\
\text { Naididae unid. spp. }\end{array}$ & 0.0 & 0.0 & 0.0 & $\begin{array}{l}\text { PHYLUM BRYOZOA } \\
\text { Class Gymnolaemata } \\
\text { Cheilostomatida unid. Spp. }\end{array}$ & 0.0 & 0.0 & 0.0 \\
\hline Oligochaeta unid. spp. & 0.0 & 0.0 & 0.0 & PHYLUM CHORDATA & & & \\
\hline Tubificidae unid. spp. & 0.0 & 0.0 & 0.0 & Class Ascidiacea & & & \\
\hline Class Polychaeta & & & & Molgula mahattensis & 0.0 & 0.0 & 0.0 \\
\hline Capitella capitata complex & 0.0 & 0.0 & 0.0 & PHYLUM CNIDARIA & & & \\
\hline Cirratulidae unid. spp. & 0.0 & 0.0 & 0.0 & Class Anthozoa & & & \\
\hline Eteone fauchaldi & 0.0 & 0.0 & 0.0 & Actiniaria - attached & 1.0 & 1.0 & 0.0 \\
\hline Eteone lighti & 0.0 & 0.0 & 0.0 & Actiniaria - burrowing & 0.0 & 1.0 & 0.0 \\
\hline Eteone unid. spp. & 0.0 & 0.0 & 0.0 & Actiniaria unid. spp. & 0.0 & 0.0 & 0.0 \\
\hline Euchone limnicola & 0.0 & 0.0 & 0.0 & Class Hydrozoa & & & \\
\hline Euchone unid. spp. & 0.0 & 0.0 & 0.0 & Hydrozoa unid spp. & 0.0 & 0.0 & 0.0 \\
\hline Exogone lourei & 0.0 & 0.0 & 0.0 & PHYLUM MOLLUSCA & & & \\
\hline Glycera unid. spp. & 0.0 & 0.0 & 0.0 & Class Bivalvia & & & \\
\hline Glycinde armigera & 0.0 & 0.0 & 0.0 & Bivalvia unid. spp. & 0.0 & 10.0 & 0.0 \\
\hline Glycinde picta & 0.0 & 0.0 & 0.0 & Gemma gemma & 0.0 & 0.0 & 0.0 \\
\hline Glycinde unid. sp. SF1 & 0.0 & 0.0 & 0.0 & Macoma petalum & 3.0 & 26.0 & 26.0 \\
\hline Glycinde unid. spp. & 0.0 & 0.0 & 0.0 & Musculista senhousia & 0.0 & 0.0 & 2.0 \\
\hline Harmothoe imbricata & 0.0 & 0.0 & 0.0 & Mya arenaria & 0.0 & 3.0 & 2.0 \\
\hline Heteromastus filiformis & 0.0 & 0.0 & 0.0 & Potamocorbula amurensis & 3.0 & $\begin{array}{l}3.0 \\
17.0\end{array}$ & $\begin{array}{l}2.0 \\
8.0\end{array}$ \\
\hline Leitoscoloplos pugettensis & 0.0 & 0.0 & 0.0 & Theora lubrica & 0.0 & 0.0 & $\begin{array}{l}0.0 \\
0.0\end{array}$ \\
\hline Maldanidae unid. spp. & 0.0 & 0.0 & 0.0 & Venerupis philippinarum & 0.0 & 0.0 & 0.0 \\
\hline Marphysa sanguinea & 0.0 & 0.0 & 0.0 & Class Gastropoda & & & \\
\hline Neanthes succinea & 4.0 & 3.0 & 0.0 & Crepidula unid. Spp. & 0.0 & 0.0 & 0.0 \\
\hline Polychaeta unid. spp. (1) & 0.0 & 0.0 & 0.0 & Gastropoda unid. sp. B & 0.0 & 0.0 & 0.0 \\
\hline Polychaeta unid. spp. (2) & 0.0 & 0.0 & 0.0 & $\begin{array}{l}\text { Gastropoda unia. sp. B } \\
\text { Ilyanassa obsoleta }\end{array}$ & 0.0 & 0.0 & 0.0 \\
\hline Polycirrus spp. & 0.0 & 0.0 & 0.0 & Philine unid. spp. & 0.0 & 0.0 & 0.0 \\
\hline Polydora cornuta & 0.0 & 0.0 & 0.0 & Urosalpinx cinerea & 0.0 & 0.0 & 0.0 \\
\hline Polydora unid. spp. & 0.0 & 0.0 & 0.0 & PHYLUM NEMATODA & & & \\
\hline Pseudopolydora kempi & 0.0 & 0.0 & 0.0 & Nematoda unid. spp. & 0.0 & 0.0 & 0.0 \\
\hline Pseudopolydora paucibranchiata & 0.0 & 0.0 & 0.0 & & & & \\
\hline Sabaco elongatus & 0.0 & 0.0 & 0.0 & PHYLUM PLATYHELIMINTHES & & & \\
\hline Sabellidae unid. spp. & 0.0 & 0.0 & 0.0 & Class Turbellaria & & & \\
\hline Sphaerosyllis californiensis & 0.0 & 0.0 & 0.0 & Turbellaria unid. sp. A & 0.0 & 0.0 & 0.0 \\
\hline Sphaerosyllis erinaceus & 0.0 & 0.0 & 0.0 & Turbellaria unid. spp. & 0.0 & 0.0 & 0.0 \\
\hline Sphaerosyllis unid. sp. A & 0.0 & 0.0 & 0.0 & & & & \\
\hline Streblospio benedicti & 0.0 & 0.0 & 0.0 & & & & \\
\hline Spionidae unid. spp. & 0.0 & 0.0 & 0.0 & & & & \\
\hline PHYLUM ARTHROPODA & & & & & & & \\
\hline Class Copepoda & & & & & & & \\
\hline Calanoida unid. spp. & 0.0 & 0.0 & 0.0 & & & & \\
\hline Harpacticoida unid. spp. & 0.0 & 0.0 & 0.0 & & & & \\
\hline Class Malacostraca & & & & & & & \\
\hline Order Amphipoda & & & & & & & \\
\hline Ampelisca abdita & 0.0 & 0.0 & 0.0 & & & & \\
\hline Ampithoe unid. spp. & 0.0 & 0.0 & 0.0 & & & & \\
\hline Caprella californica & 0.0 & 0.0 & 0.0 & & & & \\
\hline Corophium heteroceratum & 2.0 & 30.0 & 0.0 & & & & \\
\hline Corophiidae unid. spp. & 0.0 & 0.0 & 0.0 & & & & \\
\hline Grandidierella japonica & 0.0 & 0.0 & 0.0 & & & & \\
\hline Monocorophium acherusicum & 0.0 & 0.0 & 0.0 & & & & \\
\hline Monocorophium insidiosum & 0.0 & 0.0 & 0.0 & & & & \\
\hline Monocorophium unid. spp. & 0.0 & 0.0 & 0.0 & & & & \\
\hline Oedicerotidae unid spp. & 0.0 & 0.0 & 0.0 & & & & \\
\hline Order Cumacea & & & & & & & \\
\hline Nippoleucon hinumensis & 0.0 & 0.0 & 0.0 & & & & \\
\hline Order Decapoda & & & & & & & \\
\hline Crangon franciscorum & 0.0 & 0.0 & 0.0 & & & & \\
\hline Hemigrapsus oregonensis & 0.0 & 0.0 & 0.0 & & & & \\
\hline Crab unid spp. & 0.0 & 0.0 & 0.0 & & & & \\
\hline Order Isopoda & & & & & & & \\
\hline Gnorisphaeroma oregonensis & 0.0 & 0.0 & 0.0 & & & & \\
\hline Paranthura japonica & 0.0 & 0.0 & 0.0 & & & & \\
\hline Synidotea laevidorsalis & 0.0 & 0.0 & 0.0 & & & & \\
\hline Synidotea unid. spp. & 0.0 & 0.0 & 0.0 & & & & \\
\hline Class Ostracoda & & & & & & & \\
\hline Eusarsiella zostericola & 0.0 & 0.0 & 0.0 & & & & \\
\hline Cyprideis unid. spp. & 0.0 & 0.0 & 0.0 & & & & \\
\hline Class Thecostraca & & & & & & & \\
\hline Balanomorpha unid. spp. & 0.0 & 0.0 & 0.0 & & & & \\
\hline Cirripedia unid. spp. & 0.0 & 0.0 & 0.0 & & & & \\
\hline
\end{tabular}


Appendix 6. GPE3 Species List And Abundance

\begin{tabular}{|c|c|c|c|c|c|c|c|}
\hline TAXON & 27-Mar-14 & 24-Jun-14 & 24-Sep-14 & TAXON & 27-Mar-14 & 24-Jun-14 & 24-Sep-14 \\
\hline $\begin{array}{l}\text { PHYLUM ANNELIDA } \\
\text { Class Oligochaeta } \\
\text { Naididae unid. spp. }\end{array}$ & 0.0 & 0.0 & 0.0 & $\begin{array}{l}\text { PHYLUM BRYOZOA } \\
\text { Class Gymnolaemata } \\
\text { Cheilostomatida unid. Spp. }\end{array}$ & 0.0 & 0.0 & 0.0 \\
\hline Oligochaeta unid. spp. & 0.0 & 0.0 & 0.0 & PHYLUM CHORDATA & & & \\
\hline Tubificidae unid. spp. & 2.0 & 0.0 & 0.0 & Class Ascidiacea & & & \\
\hline Class Polychaeta & & & & Molgula mahattensis & 0.0 & 0.0 & 0.0 \\
\hline Capitella capitata complex & 0.0 & 0.0 & 0.0 & PHYLUM CNIDARIA & & & \\
\hline Cirratulidae unid. spp. & 0.0 & 0.0 & 0.0 & Class Anthozoa & & & \\
\hline Eteone fauchaldi & 0.0 & 0.0 & 0.0 & Actiniaria - attached & 0.0 & 0.0 & 0.0 \\
\hline Eteone lighti & 2.0 & 0.0 & 0.0 & Actiniaria - burrowing & 0.0 & 0.0 & 0.0 \\
\hline Eteone unid. spp. & 0.0 & 0.0 & 0.0 & Actiniaria unid. spp. & 0.0 & 0.0 & 0.0 \\
\hline Euchone limnicola & 0.0 & 0.0 & 0.0 & Class Hydrozoa & & & \\
\hline Euchone unid. spp. & 0.0 & 0.0 & 0.0 & Hydrozoa unid spp. & 0.0 & 0.0 & 0.0 \\
\hline Exogone lourei & 0.0 & 0.0 & 0.0 & PHYLUM MOLLUSCA & & & \\
\hline Glycera unid. spp. & 0.0 & 0.0 & 0.0 & Class Bivalvia & & & \\
\hline Glycinde armigera & 0.0 & 0.0 & 0.0 & Bivalvia unid. spp. & 0.0 & 0.0 & 0.0 \\
\hline Glycinde picta & 0.0 & 0.0 & 0.0 & Gemma gemma & 0.0 & 0.0 & 0.0 \\
\hline Glycinde unid. sp. SF1 & 0.0 & 0.0 & 0.0 & $\begin{array}{l}\text { Gemma gemma } \\
\text { Macoma petalum }\end{array}$ & 4.0 & 47.0 & 34.0 \\
\hline Glycinde unid. spp. & 0.0 & 0.0 & 0.0 & Musculista senhousia & 0.0 & 0.0 & 0.0 \\
\hline Harmothoe imbricata & 0.0 & 0.0 & 0.0 & Mya arenaria & 0.0 & 0.0 & 1.0 \\
\hline Heteromastus filiformis & 0.0 & 0.0 & 0.0 & Potamocorbula amurensis & $\begin{array}{l}0.0 \\
1.0\end{array}$ & 29.0 & $\begin{array}{l}1.0 \\
30.0\end{array}$ \\
\hline Leitoscoloplos pugettensis & 0.0 & 0.0 & 0.0 & Theora lubrica & $\begin{array}{l}1.0 \\
0.0\end{array}$ & $\begin{array}{l}29.0 \\
0.0\end{array}$ & 0.0 \\
\hline Maldanidae unid. spp. & 0.0 & 0.0 & 0.0 & Venerupis philippinarum & 0.0 & 0.0 & 0.0 \\
\hline Marphysa sanguinea & 0.0 & 0.0 & 0.0 & Class Gastropoda & & & \\
\hline Neanthes succinea & 0.0 & 7.0 & 0.0 & Crepidula unid. Spp. & 0.0 & 0.0 & 0.0 \\
\hline Polychaeta unid. spp. (1) & 0.0 & 0.0 & 0.0 & Gastropoda unid. sp. B & 0.0 & 0.0 & 0.0 \\
\hline Polychaeta unid. spp. (2) & 0.0 & 0.0 & 0.0 & llyanassa obsoleta & 0.0 & 0.0 & 0.0 \\
\hline Polycirrus spp. & 0.0 & 0.0 & 0.0 & $\begin{array}{l}\text { llyanassa obsoleta } \\
\text { Philine unid. spp. }\end{array}$ & 0.0 & 0.0 & 0.0 \\
\hline Polydora cornuta & 0.0 & 0.0 & 0.0 & Urosalpinx cinerea & 0.0 & 0.0 & 0.0 \\
\hline Polydora unid. spp. & 0.0 & 0.0 & 0.0 & PHYLUM NEMATODA & & & \\
\hline Pseudopolydora kempi & 0.0 & 0.0 & 0.0 & Nematoda unid. spp. & 0.0 & 0.0 & 0.0 \\
\hline Pseudopolydora paucibranchiata & 0.0 & 0.0 & 0.0 & PHYLUM PLATYHELMINTHES & & & \\
\hline Sabaco elongatus & 0.0 & 0.0 & 0.0 & Class Turbellaria & & & \\
\hline Sabellidae unid. spp. & 0.0 & 0.0 & $\begin{array}{l}0.0 \\
0.0\end{array}$ & Turbellaria unid. sp. A & 0.0 & 0.0 & 0.0 \\
\hline $\begin{array}{l}\text { Sphaerosyllis californiensis } \\
\text { Sphaerosyllis erinaceus }\end{array}$ & $\begin{array}{l}0.0 \\
0.0\end{array}$ & $\begin{array}{l}0.0 \\
0.0\end{array}$ & $\begin{array}{l}0.0 \\
0.0\end{array}$ & Turbellaria unid. spp. & 0.0 & 0.0 & 0.0 \\
\hline Sphaerosyllis unid. sp. A & 0.0 & 0.0 & 0.0 & & & & \\
\hline Streblospio benedicti & 0.0 & 0.0 & 0.0 & & & & \\
\hline Spionidae unid. spp. & 0.0 & 0.0 & 0.0 & & & & \\
\hline \multicolumn{8}{|l|}{ PHYLUM ARTHROPODA } \\
\hline \multicolumn{8}{|l|}{ Class Copepoda } \\
\hline Calanoida unid. spp. & 0.0 & 0.0 & 0.0 & & & & \\
\hline Harpacticoida unid. spp. & 0.0 & 0.0 & 0.0 & & & & \\
\hline \multicolumn{8}{|l|}{ Class Malacostraca } \\
\hline \multicolumn{8}{|l|}{ Order Amphipoda } \\
\hline Ampelisca abdita & 0.0 & 0.0 & 0.0 & & & & \\
\hline Ampithoe unid. spp. & 0.0 & 0.0 & 0.0 & & & & \\
\hline Caprella californica & 0.0 & 0.0 & 0.0 & & & & \\
\hline Corophium heteroceratum & 0.0 & 0.0 & 0.0 & & & & \\
\hline Corophiidae unid. spp. & 0.0 & 0.0 & 0.0 & & & & \\
\hline Grandidierella japonica & 5.0 & 0.0 & 30.0 & & & & \\
\hline Monocorophium acherusicum & 0.0 & 0.0 & 0.0 & & & & \\
\hline Monocorophium insidiosum & 0.0 & 0.0 & 0.0 & & & & \\
\hline Monocorophium unid. spp. & 0.0 & 1476.0 & 1872.0 & & & & \\
\hline Oedicerotidae unid spp. & 0.0 & 0.0 & 0.0 & & & & \\
\hline \multicolumn{8}{|l|}{ Order Cumacea } \\
\hline Nippoleucon hinumensis & 3.0 & 0.0 & 0.0 & & & & \\
\hline \multicolumn{8}{|l|}{ Order Decapoda } \\
\hline Crangon franciscorum & 0.0 & 0.0 & 0.0 & & & & \\
\hline Hemigrapsus oregonensis & 0.0 & 0.0 & 0.0 & & & & \\
\hline Crab unid spp. & 0.0 & 0.0 & 0.0 & & & & \\
\hline \multicolumn{8}{|l|}{ Order Isopoda } \\
\hline Gnorisphaeroma oregonensis & 0.0 & 0.0 & 0.0 & & & & \\
\hline Paranthura japonica & 0.0 & 0.0 & 0.0 & & & & \\
\hline Synidotea laevidorsalis & 0.0 & 3.0 & 1.0 & & & & \\
\hline Synidotea unid. spp. & 0.0 & 0.0 & 0.0 & & & & \\
\hline \multicolumn{8}{|l|}{ Class Ostracoda } \\
\hline Eusarsiella zostericola & 0.0 & 0.0 & 0.0 & & & & \\
\hline Cyprideis unid. spp. & 0.0 & 0.0 & 0.0 & & & & \\
\hline \multicolumn{8}{|l|}{ Class Thecostraca } \\
\hline Balanomorpha unid. spp. & 0.0 & 0.0 & 0.0 & & & & \\
\hline Cirripedia unid. spp. & 0.0 & 0.0 & 0.0 & & & & \\
\hline
\end{tabular}


Appendix 7. GPE4 Species List and Abundance

\begin{tabular}{|c|c|c|c|c|c|c|c|}
\hline TAXON & 27-Mar-14 & 24-Jun-14 & 24-Sep-14 & TAXON & 27-Mar-14 & 24-Jun-14 & 24-Sep-14 \\
\hline $\begin{array}{l}\text { PHYLUM ANNELIDA } \\
\text { Class Oligochaeta }\end{array}$ & & & & $\begin{array}{l}\text { PHYLUM BRYOZOA } \\
\text { Class Gymnolaemata }\end{array}$ & & & \\
\hline Naididae unid. spp. & 0.0 & 0.0 & 0.0 & Cheilostomatida unid. Spp. & 0.0 & 0.0 & 0.0 \\
\hline Oligochaeta unid. spp. & 0.0 & 0.0 & 0.0 & PHYLUM CHORDATA & & & \\
\hline Tubificidae unid. spp. & 23.0 & 0.0 & 0.0 & Class Ascidiacea & & & \\
\hline Class Polychaeta & & & & Molgula mahattensis & 0.0 & 0.0 & 0.0 \\
\hline Capitella capitata complex & 0.0 & 0.0 & 0.0 & PHYLUM CNIDARIA & & & \\
\hline Cirratulidae unid. spp. & 0.0 & 0.0 & 0.0 & Class Anthozoa & & & \\
\hline Eteone fauchaldi & 0.0 & 0.0 & 0.0 & Actiniaria - attached & 0.0 & 0.0 & 0.0 \\
\hline Eteone lighti & 0.0 & 1.0 & 0.0 & Actiniaria - burrowing & 0.0 & 0.0 & 0.0 \\
\hline Eteone unid. spp. & 0.0 & 0.0 & 0.0 & Actiniaria unid. spp. & 0.0 & 0.0 & 0.0 \\
\hline Euchone limnicola & 0.0 & 0.0 & 0.0 & Class Hydrozoa & & & \\
\hline Euchone unid. spp. & 0.0 & 0.0 & 0.0 & Hydrozoa unid spp. & 0.0 & 0.0 & 0.0 \\
\hline Exogone lourei & 0.0 & 0.0 & 0.0 & PHYLUM MOLLUSCA & & & \\
\hline Glycera unid. spp. & 0.0 & 0.0 & 0.0 & Class Bivalvia & & & \\
\hline Glycinde armigera & 0.0 & 0.0 & 0.0 & Bivalvia unid. spp. & 0.0 & 0.0 & 0.0 \\
\hline Glycinde picta & 0.0 & 0.0 & 0.0 & Gemma gemma & 0.0 & 0.0 & 0.0 \\
\hline Glycinde unid. sp. SF1 & 0.0 & 0.0 & 0.0 & Macoma petalum & 2.0 & 78.0 & 29.0 \\
\hline Glycinde unid. spp. & 0.0 & 0.0 & 0.0 & Musculista senhousia & 0.0 & 0.0 & 0.0 \\
\hline Harmothoe imbricata & 0.0 & 0.0 & 0.0 & Mya arenaria & 0.0 & 0.0 & 0.0 \\
\hline Heteromastus filiformis & 0.0 & 0.0 & 0.0 & Potamocorbula amurensis & 1.0 & 2.0 & 8.0 \\
\hline Leitoscoloplos pugettensis & 0.0 & 0.0 & 0.0 & Theora lubrica & 0.0 & 0.0 & 0.0 \\
\hline Maldanidae unid. spp. & 0.0 & 0.0 & 0.0 & Venerupis philippinarum & 0.0 & 0.0 & 0.0 \\
\hline Marphysa sanguinea & 0.0 & 0.0 & 0.0 & $\begin{array}{l}\text { Class Gastropoda } \\
\text { Clis philpin }\end{array}$ & & 0.0 & \\
\hline Neanthes succinea & 0.0 & 3.0 & 0.0 & $\begin{array}{l}\text { Crepsidula unid. Spp. } \\
\text { Creptropoda }\end{array}$ & 0.0 & 0.0 & 0.0 \\
\hline Polychaeta unid. spp. (1) & 0.0 & 0.0 & 0.0 & Gastropoda unid. sp. B & 0.0 & 0.0 & 0.0 \\
\hline Polychaeta unid. spp. (2) & 0.0 & 0.0 & 0.0 & llyanassa obsoleta & 0.0 & 0.0 & 0.0 \\
\hline Polycirrus spp. & 0.0 & 0.0 & 0.0 & Philine unid. spp. & 0.0 & 0.0 & 0.0 \\
\hline Polydora cornuta & 0.0 & 0.0 & 0.0 & Urosalpinx cinerea & 0.0 & 0.0 & 0.0 \\
\hline Polydora unid. spp. & 0.0 & 0.0 & 0.0 & PHYLUM NEMATODA & & & \\
\hline Pseudopolydora kempi & 0.0 & 0.0 & 0.0 & PHYYUM NEMAIODA & & & \\
\hline Pseudopolydora paucibranchiata & 0.0 & 0.0 & 0.0 & Nematoda unid. spp. & 0.0 & 0.0 & 0.0 \\
\hline Sabaco elongatus & 0.0 & 0.0 & 0.0 & PHYLUM PLATYHELMINTHES & & & \\
\hline Sabellidae unid. spp. & 0.0 & 0.0 & 0.0 & Class Turbellaria & & & \\
\hline Sphaerosyllis californiensis & 0.0 & 0.0 & 0.0 & Turbellaria unid. sp. A & 0.0 & 0.0 & 0.0 \\
\hline Sphaerosyllis erinaceus & 0.0 & 0.0 & 0.0 & Turbellaria unid. spp. & 0.0 & 0.0 & 0.0 \\
\hline Sphaerosyllis unid. sp. A & 0.0 & 0.0 & 0.0 & & & & \\
\hline Streblospio benedicti & 0.0 & 0.0 & 0.0 & & & & \\
\hline Spionidae unid. spp. & 0.0 & 0.0 & 0.0 & & & & \\
\hline \multicolumn{8}{|l|}{ PHYLUM ARTHROPODA } \\
\hline \multicolumn{8}{|l|}{ Class Copepoda } \\
\hline Calanoida unid. spp. & 0.0 & 0.0 & 0.0 & & & & \\
\hline Harpacticoida unid. spp. & 0.0 & 0.0 & 0.0 & & & & \\
\hline \multicolumn{8}{|l|}{ Class Malacostraca } \\
\hline \multicolumn{8}{|l|}{ Order Amphipoda } \\
\hline Ampelisca abdita & 0.0 & 0.0 & 0.0 & & & & \\
\hline Ampithoe unid. spp. & 0.0 & 0.0 & 0.0 & & & & \\
\hline Caprella californica & 0.0 & 0.0 & 0.0 & & & & \\
\hline Corophium heteroceratum & 7.0 & 0.0 & 0.0 & & & & \\
\hline Corophiidae unid. spp. & 0.0 & 0.0 & 0.0 & & & & \\
\hline Grandidierella japonica & 4.0 & 3.0 & 120.0 & & & & \\
\hline Monocorophium acherusicum & 0.0 & 0.0 & 0.0 & & & & \\
\hline Monocorophium insidiosum & 0.0 & 0.0 & 0.0 & & & & \\
\hline Monocorophium unid. spp. & 0.0 & 28.0 & 521.0 & & & & \\
\hline Oedicerotidae unid spp. & 0.0 & 0.0 & 0.0 & & & & \\
\hline \multicolumn{8}{|l|}{ Order Cumacea } \\
\hline Nippoleucon hinumensis & 0.0 & 5.0 & 0.0 & & & & \\
\hline \multicolumn{8}{|l|}{ Order Decapoda } \\
\hline Crangon franciscorum & 0.0 & 0.0 & 0.0 & & & & \\
\hline Hemigrapsus oregonensis & 0.0 & 0.0 & 0.0 & & & & \\
\hline Crab unid spp. & 0.0 & 0.0 & 0.0 & & & & \\
\hline \multicolumn{8}{|l|}{ Order Isopoda } \\
\hline Gnorisphaeroma oregonensis & 0.0 & 0.0 & 0.0 & & & & \\
\hline Paranthura japonica & 0.0 & 0.0 & 0.0 & & & & \\
\hline Synidotea laevidorsalis & 0.0 & 0.0 & 0.0 & & & & \\
\hline Synidotea unid. spp. & 0.0 & 0.0 & 0.0 & & & & \\
\hline \multicolumn{8}{|l|}{ Class Ostracoda } \\
\hline Eusarsiella zostericola & 0.0 & 0.0 & 0.0 & & & & \\
\hline Cyprideis unid. spp. & 0.0 & 0.0 & 0.0 & & & & \\
\hline \multicolumn{8}{|l|}{ Class Thecostraca } \\
\hline Balanomorpha unid. spp. & 0.0 & 0.0 & 0.0 & & & & \\
\hline Cirripedia unid. spp. & 0.0 & 0.0 & 0.0 & & & & \\
\hline
\end{tabular}


Appendix 8. SB13 Species List and Abundance

\begin{tabular}{|c|c|c|c|c|c|c|c|}
\hline TAXON & 27-Mar-14 & 24-Jun-14 & 24-Sep-14 & TAXON & 27-Mar-14 & 24-Jun-14 & 24-Sep-14 \\
\hline $\begin{array}{l}\text { PHYLUM ANNELIDA } \\
\text { Class Oligochaeta }\end{array}$ & & & & $\begin{array}{l}\text { PHYLUM BRYOZOA } \\
\text { Class Gymnolaemata }\end{array}$ & & & \\
\hline Naididae unid. spp. & 0.0 & 0.0 & 0.0 & Cheilostomatida unid. Spp. & 0.0 & 0.0 & 0.0 \\
\hline Oligochaeta unid. spp. & 0.0 & 0.0 & 0.0 & PHYLUM CHORDATA & & & \\
\hline Tubificidae unid. spp. & 0.0 & 0.0 & 0.0 & Class Ascidiacea & & & \\
\hline Class Polychaeta & & & & Molgula mahattensis & 0.0 & 0.0 & 0.0 \\
\hline Capitella capitata complex & 0.0 & 0.0 & 0.0 & PHYLUM CNIDARIA & & & \\
\hline Cirratulidae unid. spp. & 0.0 & 0.0 & 0.0 & Class Anthozoa & & & \\
\hline Eteone fauchaldi & 0.0 & 0.0 & 0.0 & Actiniaria - attached & 0.0 & 0.0 & 0.0 \\
\hline Eteone lighti & 0.0 & 0.0 & 0.0 & Actiniaria - burrowing & 0.0 & 0.0 & 0.0 \\
\hline Eteone unid. spp. & 0.0 & 0.0 & 0.0 & Actiniaria unid. spp. & 0.0 & 0.0 & 0.0 \\
\hline Euchone limnicola & 0.0 & 0.0 & 0.0 & Class Hydrozoa & & & \\
\hline Euchone unid. spp. & 0.0 & 0.0 & 0.0 & Hydrozoa unid spp. & 0.0 & 0.0 & 0.0 \\
\hline Exogone lourei & 0.0 & 0.0 & 0.0 & PHYLUM MOLLUSCA & & & \\
\hline Glycera unid. spp. & 0.0 & 0.0 & 0.0 & Class Bivalvia & & & \\
\hline Glycinde armigera & 0.0 & 0.0 & 0.0 & Bivalvia unid. spp. & 0.0 & 0.0 & 0.0 \\
\hline Glycinde picta & 0.0 & 0.0 & 0.0 & Gemma gemma & 0.0 & 0.0 & 0.0 \\
\hline Glycinde unid. sp. SF1 & 0.0 & 0.0 & 0.0 & Macoma petalum & 0.0 & 0.0 & 0.0 \\
\hline Glycinde unid. spp. & 0.0 & 0.0 & 0.0 & Musculista senhousia & 0.0 & 0.0 & 0.0 \\
\hline Harmothoe imbricata & 0.0 & 0.0 & 0.0 & Mya arenaria & 0.0 & 0.0 & 0.0 \\
\hline Heteromastus filiformis & 0.0 & 0.0 & 0.0 & Potamocorbula amurensis & 6.0 & 1.0 & 0.0 \\
\hline Leitoscoloplos pugettensis & 0.0 & 0.0 & 0.0 & Theora lubrica & 0.0 & 0.0 & 0.0 \\
\hline Maldanidae unid. spp. & 0.0 & 0.0 & 0.0 & Venerupis philippinarum & 0.0 & 0.0 & 0.0 \\
\hline Marphysa sanguinea & 0.0 & 0.0 & 0.0 & $\begin{array}{l}\text { Class Gastropoda } \\
\text { Clis philpin }\end{array}$ & & 0.0 & \\
\hline Neanthes succinea & 4.0 & 0.0 & 0.0 & $\begin{array}{l}\text { Crepsidula unid. Spp. } \\
\text { Creptropoda }\end{array}$ & 0.0 & 0.0 & 0.0 \\
\hline Polychaeta unid. spp. (1) & 0.0 & 0.0 & 0.0 & Gastropoda unid. sp. B & 0.0 & 0.0 & 0.0 \\
\hline Polychaeta unid. spp. (2) & 0.0 & 0.0 & 0.0 & llyanassa obsoleta & 0.0 & 0.0 & 0.0 \\
\hline Polycirrus spp. & 0.0 & 0.0 & 0.0 & Philine unid. spp. & 0.0 & 0.0 & 0.0 \\
\hline Polydora cornuta & 0.0 & 0.0 & 0.0 & Urosalpinx cinerea & 0.0 & 0.0 & 0.0 \\
\hline Polydora unid. spp. & 0.0 & 0.0 & 0.0 & PHYLUM NEMATODA & & & \\
\hline Pseudopolydora kempi & 0.0 & 0.0 & 0.0 & PHYYUM NEMAIODA & & & \\
\hline Pseudopolydora paucibranchiata & 0.0 & 0.0 & 0.0 & Nematoda unid. spp. & 0.0 & 0.0 & 0.0 \\
\hline Sabaco elongatus & 0.0 & 0.0 & 0.0 & PHYLUM PLATYHELMINTHES & & & \\
\hline Sabellidae unid. spp. & 0.0 & 0.0 & 0.0 & Class Turbellaria & & & \\
\hline Sphaerosyllis californiensis & 0.0 & 0.0 & 0.0 & Turbellaria unid. sp. A & 0.0 & 0.0 & 0.0 \\
\hline Sphaerosyllis erinaceus & 0.0 & 0.0 & 0.0 & Turbellaria unid. spp. & 0.0 & 0.0 & 0.0 \\
\hline Sphaerosyllis unid. sp. A & 0.0 & 0.0 & 0.0 & & & & \\
\hline Streblospio benedicti & 0.0 & 0.0 & 0.0 & & & & \\
\hline Spionidae unid. spp. & 0.0 & 0.0 & 0.0 & & & & \\
\hline \multicolumn{8}{|l|}{ PHYLUM ARTHROPODA } \\
\hline \multicolumn{8}{|l|}{ Class Copepoda } \\
\hline Calanoida unid. spp. & 0.0 & 0.0 & 0.0 & & & & \\
\hline Harpacticoida unid. spp. & 0.0 & 0.0 & 0.0 & & & & \\
\hline \multicolumn{8}{|l|}{ Class Malacostraca } \\
\hline \multicolumn{8}{|l|}{ Order Amphipoda } \\
\hline Ampelisca abdita & 0.0 & 0.0 & 0.0 & & & & \\
\hline Ampithoe unid. spp. & 0.0 & 0.0 & 0.0 & & & & \\
\hline Caprella californica & 0.0 & 0.0 & 0.0 & & & & \\
\hline Corophium heteroceratum & 0.0 & 0.0 & 0.0 & & & & \\
\hline Corophiidae unid. spp. & 0.0 & 0.0 & 0.0 & & & & \\
\hline Grandidierella japonica & 0.0 & 0.0 & 0.0 & & & & \\
\hline Monocorophium acherusicum & 0.0 & 0.0 & 0.0 & & & & \\
\hline Monocorophium insidiosum & 0.0 & 0.0 & 0.0 & & & & \\
\hline Monocorophium unid. spp. & 18.0 & 0.0 & 45.0 & & & & \\
\hline Oedicerotidae unid spp. & 0.0 & 0.0 & 0.0 & & & & \\
\hline \multicolumn{8}{|l|}{ Order Cumacea } \\
\hline Nippoleucon hinumensis & 0.0 & 0.0 & 0.0 & & & & \\
\hline \multicolumn{8}{|l|}{ Order Decapoda } \\
\hline Crangon franciscorum & 0.0 & 0.0 & 0.0 & & & & \\
\hline Hemigrapsus oregonensis & 0.0 & 0.0 & 0.0 & & & & \\
\hline Crab unid spp. & 0.0 & 0.0 & 0.0 & & & & \\
\hline \multicolumn{8}{|l|}{ Order Isopoda } \\
\hline Gnorisphaeroma oregonensis & 0.0 & 0.0 & 0.0 & & & & \\
\hline Paranthura japonica & 0.0 & 0.0 & 0.0 & & & & \\
\hline Synidotea laevidorsalis & 0.0 & 3.0 & 0.0 & & & & \\
\hline Synidotea unid. spp. & 0.0 & 0.0 & 0.0 & & & & \\
\hline \multicolumn{8}{|l|}{ Class Ostracoda } \\
\hline Eusarsiella zostericola & 0.0 & 0.0 & 0.0 & & & & \\
\hline Cyprideis unid. spp. & 0.0 & 0.0 & 0.0 & & & & \\
\hline \multicolumn{8}{|l|}{ Class Thecostraca } \\
\hline Balanomorpha unid. spp. & 0.0 & 0.0 & 0.0 & & & & \\
\hline Cirripedia unid. spp. & 0.0 & 0.0 & 0.0 & & & & \\
\hline
\end{tabular}


Appendix 9. SB14 Species List and Abundance

\begin{tabular}{|c|c|c|c|c|c|c|c|}
\hline TAXON & 27-Mar-14 & 24-Jun-14 & 24-Sep-14 & TAXON & 27-Mar-14 & 24-Jun-14 & 24-Sep-14 \\
\hline $\begin{array}{l}\text { PHYLUM ANNELIDA } \\
\text { Class Oligochaeta }\end{array}$ & & & & $\begin{array}{l}\text { PHYLUM BRYOZOA } \\
\text { Class Gymnolaemata }\end{array}$ & & & \\
\hline Naididae unid. spp. & 0.0 & 0.0 & 0.0 & Cheilostomatida unid. Spp. & 0.0 & 0.0 & 0.0 \\
\hline Oligochaeta unid. spp. & 0.0 & 0.0 & 0.0 & PHYLUM CHORDATA & & & \\
\hline Tubificidae unid. spp. & 0.0 & 0.0 & 0.0 & Class Ascidiacea & & & \\
\hline Class Polychaeta & & & & Molgula mahattensis & 0.0 & 0.0 & 0.0 \\
\hline Capitella capitata complex & 0.0 & 0.0 & 0.0 & PHYLUM CNIDARIA & & & \\
\hline Cirratulidae unid. spp. & 0.0 & 0.0 & 0.0 & Class Anthozoa & & & \\
\hline Eteone fauchaldi & 0.0 & 0.0 & 0.0 & Actiniaria - attached & 0.0 & 0.0 & 0.0 \\
\hline Eteone lighti & 0.0 & 0.0 & 0.0 & Actiniaria - burrowing & 0.0 & 5.0 & 0.0 \\
\hline Eteone unid. spp. & 0.0 & 0.0 & 0.0 & Actiniaria unid. spp. & 0.0 & 0.0 & 0.0 \\
\hline Euchone limnicola & 0.0 & 0.0 & 0.0 & Class Hydrozoa & & & \\
\hline Exogone lourei & 0.0 & 0.0 & 0.0 & Hydrozoa unid spp. & 0.0 & 0.0 & 0.0 \\
\hline Euchone unid. spp. & 0.0 & 0.0 & 0.0 & PHYLUM MOLLUSCA & & & \\
\hline Glycera unid. spp. & 0.0 & 0.0 & 0.0 & Class Bivalvia & & & \\
\hline Glycinde armigera & 0.0 & 0.0 & 0.0 & Bivalvia unid. spp. & 0.0 & 0.0 & 0.0 \\
\hline Glycinde picta & 0.0 & 0.0 & 0.0 & Gemma gemma & 0.0 & 0.0 & 0.0 \\
\hline Glycinde unid. sp. SF1 & 0.0 & 0.0 & 0.0 & Macoma petalum & 3.0 & 1.0 & 3.0 \\
\hline Glycinde unid. spp. & 0.0 & 0.0 & 0.0 & Musculista senhousia & 0.0 & 0.0 & 0.0 \\
\hline Harmothoe imbricata & 0.0 & 0.0 & 0.0 & Mya arenaria & 0.0 & 0.0 & 0.0 \\
\hline Heteromastus filiformis & 0.0 & 0.0 & 0.0 & Potamocorbula amurensis & 0.0 & 0.0 & 0.0 \\
\hline Leitoscoloplos pugettensis & 0.0 & 0.0 & 0.0 & Theora lubrica & 0.0 & 0.0 & 0.0 \\
\hline Maldanidae unid. spp. & 0.0 & 0.0 & 0.0 & Venerupis philippinarum & 0.0 & 0.0 & 0.0 \\
\hline Marphysa sanguinea & 0.0 & 0.0 & 0.0 & $\begin{array}{l}\text { Class Gastropoda } \\
\text { Clis philpin }\end{array}$ & & 0.0 & \\
\hline Neanthes succinea & 0.0 & 1.0 & 4.0 & Crepidula unid. Spp. & 0.0 & 0.0 & 0.0 \\
\hline Polychaeta unid. spp. (1) & 0.0 & 0.0 & 0.0 & Gastropoda unid. sp. B & 0.0 & 0.0 & 0.0 \\
\hline Polychaeta unid. spp. (2) & 0.0 & 0.0 & 0.0 & llyanassa obsoleta & 0.0 & 0.0 & 0.0 \\
\hline Polycirrus spp. & 0.0 & 0.0 & 0.0 & Philine unid. spp. & 0.0 & 0.0 & 0.0 \\
\hline Polydora cornuta & 0.0 & 0.0 & 0.0 & Urosalpinx cinerea & 0.0 & 0.0 & 0.0 \\
\hline Polydora unid. spp. & 0.0 & 0.0 & 0.0 & PHYLUM NEMATODA & & & \\
\hline Pseudopolydora kempi & 0.0 & 0.0 & 0.0 & PHYYUM NEMAIODA & & & \\
\hline Pseudopolydora paucibranchiata & 0.0 & 0.0 & 0.0 & Nematoda unid. spp. & 0.0 & 0.0 & 0.0 \\
\hline Sabaco elongatus & 0.0 & 0.0 & 0.0 & PHYLUM PLATYHELMINTHES & & & \\
\hline Sabellidae unid. spp. & 0.0 & 4.0 & 0.0 & Class Turbellaria & & & \\
\hline Sphaerosyllis californiensis & 0.0 & 0.0 & 0.0 & Turbellaria unid. sp. A & 0.0 & 0.0 & 0.0 \\
\hline Sphaerosyllis erinaceus & 0.0 & 0.0 & 0.0 & Turbellaria unid. spp. & 0.0 & 0.0 & 0.0 \\
\hline Sphaerosyllis unid. sp. A & 0.0 & 0.0 & 0.0 & & & & \\
\hline Streblospio benedicti & 0.0 & 0.0 & 0.0 & & & & \\
\hline Spionidae unid. spp. & 0.0 & 0.0 & 0.0 & & & & \\
\hline \multicolumn{8}{|l|}{ PHYLUM ARTHROPODA } \\
\hline \multicolumn{8}{|l|}{ Class Copepoda } \\
\hline Calanoida unid. spp. & 0.0 & 0.0 & 0.0 & & & & \\
\hline Harpacticoida unid. spp. & 0.0 & 0.0 & 0.0 & & & & \\
\hline \multicolumn{8}{|l|}{ Class Malacostraca } \\
\hline \multicolumn{8}{|l|}{ Order Amphipoda } \\
\hline Ampelisca abdita & 0.0 & 0.0 & 0.0 & & & & \\
\hline Ampithoe unid. spp. & 0.0 & 0.0 & 0.0 & & & & \\
\hline Caprella californica & 0.0 & 0.0 & 0.0 & & & & \\
\hline Corophium heteroceratum & 0.0 & 0.0 & 0.0 & & & & \\
\hline Corophiidae unid. spp. & 0.0 & 0.0 & 0.0 & & & & \\
\hline Grandidierella japonica & 20.0 & 2.0 & 25.0 & & & & \\
\hline Monocorophium acherusicum & 0.0 & 0.0 & 0.0 & & & & \\
\hline Monocorophium insidiosum & 0.0 & 0.0 & 0.0 & & & & \\
\hline Monocorophium unid. spp. & 872.0 & 2.0 & 461.0 & & & & \\
\hline Oedicerotidae unid spp. & 0.0 & 0.0 & 0.0 & & & & \\
\hline \multicolumn{8}{|l|}{ Order Cumacea } \\
\hline Nippoleucon hinumensis & 0.0 & 0.0 & 0.0 & & & & \\
\hline \multicolumn{8}{|l|}{ Order Decapoda } \\
\hline Crangon franciscorum & 0.0 & 0.0 & 0.0 & & & & \\
\hline Hemigrapsus oregonensis & 0.0 & 0.0 & 0.0 & & & & \\
\hline Crab unid spp. & 0.0 & 0.0 & 0.0 & & & & \\
\hline \multicolumn{8}{|l|}{ Order Isopoda } \\
\hline Gnorisphaeroma oregonensis & 0.0 & 0.0 & 0.0 & & & & \\
\hline Paranthura japonica & 0.0 & 0.0 & 0.0 & & & & \\
\hline Synidotea laevidorsalis & 0.0 & 0.0 & 0.0 & & & & \\
\hline Synidotea unid. spp. & 0.0 & 0.0 & 0.0 & & & & \\
\hline \multicolumn{8}{|l|}{ Class Ostracoda } \\
\hline Eusarsiella zostericola & 0.0 & 0.0 & 0.0 & & & & \\
\hline Cyprideis unid. spp. & 0.0 & 0.0 & 0.0 & & & & \\
\hline \multicolumn{8}{|l|}{ Class Thecostraca } \\
\hline Balanomorpha unid. spp. & 0.0 & 0.0 & 0.0 & & & & \\
\hline Cirripedia unid. spp. & 0.0 & 0.0 & 0.0 & & & & \\
\hline
\end{tabular}


Appendix 10. SB15 Species List and Abundance

\begin{tabular}{|c|c|c|c|c|c|c|c|}
\hline TAXON & 27-Mar-14 & 24-Jun-14 & 24-Sep-14 & TAXON & 27-Mar-14 & 24-Jun-14 & 24-Sep-14 \\
\hline $\begin{array}{l}\text { PHYLUM ANNELIDA } \\
\text { Class Oligochaeta }\end{array}$ & & & & $\begin{array}{l}\text { PHYLUM BRYOZOA } \\
\text { Class Gymnolaemata }\end{array}$ & & & \\
\hline Naididae unid. spp. & 0.0 & 0.0 & 0.0 & Cheilostomatida unid. Spp. & 0.0 & 0.0 & 0.0 \\
\hline Oligochaeta unid. spp. & 0.0 & 0.0 & 0.0 & PHYLUM CHORDATA & & & \\
\hline Tubificidae unid. spp. & 0.0 & 3.0 & 0.0 & Class Ascidiacea & & & \\
\hline Class Polychaeta & & & & Molgula mahattensis & 0.0 & 0.0 & 0.0 \\
\hline Capitella capitata complex & 0.0 & 6.0 & 0.0 & PHYLUM CNIDARIA & & & \\
\hline Cirratulidae unid. spp. & 0.0 & 0.0 & 0.0 & Class Anthozoa & & & \\
\hline Etone fauchaldi & 0.0 & 0.0 & 0.0 & Actiniaria - attached & 0.0 & 0.0 & 0.0 \\
\hline Eteone lighti & 0.0 & 0.0 & 0.0 & Actiniaria - burrowing & 0.0 & 0.0 & 0.0 \\
\hline Eteone unid. spp. & 0.0 & 0.0 & 0.0 & Actiniaria unid. spp. & 0.0 & 0.0 & 0.0 \\
\hline Euchone limnicola & 0.0 & 0.0 & 0.0 & Class Hydrozoa & & & \\
\hline Euchone unid. spp. & 0.0 & 0.0 & 0.0 & Hydrozoa unid spp. & 0.0 & 0.0 & 0.0 \\
\hline Exogone lourei & 0.0 & 0.0 & 0.0 & PHYLUM MOLLUSCA & & & \\
\hline Glycera unid. spp. & 0.0 & 0.0 & 0.0 & Class Bivalvia & & & \\
\hline Glycinde armigera & 0.0 & 0.0 & 0.0 & Bivalvia unid. spp. & 0.0 & 0.0 & 0.0 \\
\hline Glycinde picta & 0.0 & 0.0 & 0.0 & Gemma gemma & 0.0 & 0.0 & 0.0 \\
\hline Glycinde unid. sp. SF1 & 0.0 & 0.0 & 0.0 & Macoma petalum & 0.0 & 0.0 & 0.0 \\
\hline Glycinde unid. spp. & 0.0 & 0.0 & 0.0 & Musculista senhousia & 0.0 & 0.0 & 0.0 \\
\hline Harmothoe imbricata & 0.0 & 0.0 & 0.0 & Mya arenaria & 0.0 & 0.0 & 0.0 \\
\hline Heteromastus filiformis & 0.0 & 0.0 & 0.0 & Potamocorbula amurensis & 0.0 & 0.0 & 0.0 \\
\hline Leitoscoloplos pugettensis & 0.0 & 0.0 & 0.0 & Theora lubrica & 0.0 & 0.0 & 0.0 \\
\hline Maldanidae unid. spp. & 0.0 & 0.0 & 0.0 & Venerupis philippinarum & 0.0 & 0.0 & 0.0 \\
\hline Marphysa sanguinea & 0.0 & 0.0 & 0.0 & $\begin{array}{l}\text { Class Gastropoda } \\
\text { Clis philpin }\end{array}$ & & 0.0 & \\
\hline Neanthes succinea & 0.0 & 0.0 & 0.0 & Crepidula unid. Spp. & 0.0 & 0.0 & 0.0 \\
\hline Polychaeta unid. spp. (1) & 0.0 & 0.0 & 0.0 & Gastropoda unid. sp. B & 0.0 & 0.0 & 0.0 \\
\hline Polychaeta unid. spp. (2) & 0.0 & 0.0 & 0.0 & llyanassa obsoleta & 0.0 & 0.0 & 0.0 \\
\hline Polycirrus spp. & 0.0 & 0.0 & 0.0 & Philine unid. spp. & 0.0 & 0.0 & 0.0 \\
\hline Polydora cornuta & 0.0 & 0.0 & 0.0 & Urosalpinx cinerea & 0.0 & 0.0 & 0.0 \\
\hline Polydora unid. spp. & 0.0 & 0.0 & 0.0 & PHYLUM NEMATODA & & & \\
\hline Pseudopolydora kempi & 0.0 & 0.0 & 0.0 & PHYYUM NEMAIODA & & & \\
\hline Pseudopolydora paucibranchiata & 0.0 & 0.0 & 0.0 & Nematoda unid. spp. & 0.0 & 0.0 & 0.0 \\
\hline Sabaco elongatus & 0.0 & 0.0 & 0.0 & PHYLUM PLATYHELMINTHES & & & \\
\hline Sabellidae unid. spp. & 0.0 & 0.0 & 0.0 & Class Turbellaria & & & \\
\hline Sphaerosyllis californiensis & 0.0 & 0.0 & 0.0 & Turbellaria unid. sp. A & 0.0 & 0.0 & 0.0 \\
\hline Sphaerosyllis erinaceus & 0.0 & 0.0 & 0.0 & Turbellaria unid. spp. & 0.0 & 0.0 & 0.0 \\
\hline Sphaerosyllis unid. sp. A & 0.0 & 0.0 & 0.0 & & & & \\
\hline Streblospio benedicti & 0.0 & 0.0 & 0.0 & & & & \\
\hline Spionidae unid. spp. & 0.0 & 0.0 & 0.0 & & & & \\
\hline \multicolumn{8}{|l|}{ PHYLUM ARTHROPODA } \\
\hline \multicolumn{8}{|l|}{ Class Copepoda } \\
\hline Calanoida unid. spp. & 0.0 & 0.0 & 0.0 & & & & \\
\hline Harpacticoida unid. spp. & 0.0 & 0.0 & 0.0 & & & & \\
\hline \multicolumn{8}{|l|}{ Class Malacostraca } \\
\hline \multicolumn{8}{|l|}{ Order Amphipoda } \\
\hline Ampelisca abdita & 22.0 & 0.0 & 0.0 & & & & \\
\hline Ampithoe unid. spp. & 0.0 & 0.0 & 0.0 & & & & \\
\hline Caprella californica & 0.0 & 0.0 & 0.0 & & & & \\
\hline Corophium heteroceratum & 0.0 & 0.0 & 0.0 & & & & \\
\hline Corophiidae unid. spp. & 0.0 & 0.0 & 0.0 & & & & \\
\hline Grandidierella japonica & 0.0 & 0.0 & 0.0 & & & & \\
\hline Monocorophium acherusicum & 0.0 & 0.0 & 0.0 & & & & \\
\hline Monocorophium insidiosum & 0.0 & 0.0 & 0.0 & & & & \\
\hline Monocorophium unid. spp. & 1683.0 & 40.0 & 67.0 & & & & \\
\hline Oedicerotidae unid spp. & 0.0 & 0.0 & 0.0 & & & & \\
\hline \multicolumn{8}{|l|}{ Order Cumacea } \\
\hline Nippoleucon hinumensis & 1.0 & 0.0 & 0.0 & & & & \\
\hline \multicolumn{8}{|l|}{ Order Decapoda } \\
\hline Crangon franciscorum & 0.0 & 1.0 & 0.0 & & & & \\
\hline Hemigrapsus oregonensis & 0.0 & 0.0 & 0.0 & & & & \\
\hline Crab unid spp. & 0.0 & 0.0 & 0.0 & & & & \\
\hline \multicolumn{8}{|l|}{ Order Isopoda } \\
\hline Gnorisphaeroma oregonensis & 1.0 & 0.0 & 0.0 & & & & \\
\hline Paranthura japonica & 0.0 & 0.0 & 0.0 & & & & \\
\hline Synidotea laevidorsalis & 0.0 & 0.0 & 0.0 & & & & \\
\hline Synidotea unid. spp. & 0.0 & 0.0 & 0.0 & & & & \\
\hline \multicolumn{8}{|l|}{ Class Ostracoda } \\
\hline Eusarsiella zostericola & 0.0 & 0.0 & 0.0 & & & & \\
\hline Cyprideis unid. spp. & 0.0 & 0.0 & 0.0 & & & & \\
\hline \multicolumn{8}{|l|}{ Class Thecostraca } \\
\hline Balanomorpha unid. spp. & 0.0 & 0.0 & 0.0 & & & & \\
\hline Cirripedia unid. spp. & 0.0 & 0.0 & 0.0 & & & & \\
\hline
\end{tabular}


Appendix 11. CC1 Species List and Abundance

\begin{tabular}{|c|c|c|c|c|c|c|c|}
\hline TAXON & 28-Jan-14 & 27-Feb-14 & 27-Mar-14 & TAXON & 28-Jan-14 & 27-Feb-14 & 27-Mar-14 \\
\hline $\begin{array}{l}\text { PHYLUM ANNELIDA } \\
\text { Class Oligochaeta }\end{array}$ & & & & $\begin{array}{l}\text { PHYLUM BRYOZOA } \\
\text { Class Gymnolaemata }\end{array}$ & \multirow[b]{2}{*}{0.0} & \multirow[b]{2}{*}{0.0} & \multirow[b]{2}{*}{0.0} \\
\hline Naididae unid. spp. & 0.0 & 0.0 & 0.0 & Cheilostomatida unid. Spp. & & & \\
\hline Oligochaeta unid. spp. & 0.0 & 0.0 & 0.0 & PHYLUM CHORDATA & \multirow[b]{3}{*}{0.0} & \multirow[b]{3}{*}{0.0} & \multirow[b]{3}{*}{0.0} \\
\hline Tubificidae unid. spp. & 0.0 & 0.0 & 0.0 & Class Ascidiacea & & & \\
\hline Class Polychaeta & & & & Molgula mahattensis & & & \\
\hline Capitella capitata complex & 0.0 & 0.0 & 0.0 & PHYLUM CNIDARIA & \multirow[b]{3}{*}{0.0} & \multirow[b]{3}{*}{0.0} & \multirow[b]{3}{*}{0.0} \\
\hline Cirratulidae unid. spp. & 0.0 & 0.0 & 0.0 & Class Anthozoa & & & \\
\hline Eteone fauchaldi & 0.0 & 0.0 & 0.0 & Actiniaria - attached & & & \\
\hline Eteone lighti & 2.0 & 1.0 & 0.0 & Actiniaria - burrowing & 0.0 & 0.0 & 0.0 \\
\hline Eteone unid. spp. & 0.0 & 0.0 & 0.0 & Actiniaria unid. spp. & 0.0 & 0.0 & 0.0 \\
\hline Euchone limnicola & 0.0 & 0.0 & 0.0 & Class Hydrozoa & & & \\
\hline Euchone unid. spp. & 0.0 & 0.0 & 0.0 & Hydrozoa unid spp. & 0.0 & 0.0 & 0.0 \\
\hline Exogone lourei & 0.0 & 0.0 & 0.0 & PHYLUM MOLLUSCA & & & \\
\hline Glycera unid. spp. & 0.0 & 0.0 & 0.0 & Class Bivalvia & & & \\
\hline Glycinde armigera & 0.0 & 0.0 & 0.0 & Bivalvia unid. spp. & 0.0 & 0.0 & 0.0 \\
\hline Glycinde picta & 0.0 & 0.0 & 0.0 & Gemma gemma & 1.0 & 2.0 & 0.0 \\
\hline Glycinde unid. sp. SF1 & 0.0 & 0.0 & 0.0 & Macoma petalum & 4.0 & 1.0 & 0.0 \\
\hline Glycinde unid. spp. & 0.0 & 0.0 & 0.0 & Musculista senhousia & 0.0 & 0.0 & 0.0 \\
\hline Harmothoe imbricata & 0.0 & 0.0 & 0.0 & Mya arenaria & 0.0 & 0.0 & 0.0 \\
\hline Heteromastus filiformis & 0.0 & 1.0 & 0.0 & Potamocorbula amurensis & 31.0 & 13.0 & 3.0 \\
\hline Leitoscoloplos pugettensis & 0.0 & 0.0 & 0.0 & Theora lubrica & 0.0 & $\begin{array}{c}130 \\
0.0\end{array}$ & 0.0 \\
\hline Maldanidae unid. spp. & 0.0 & 0.0 & 0.0 & Venerupis philippinarum & 0.0 & 0.0 & 0.0 \\
\hline Marphysa sanguinea & 0.0 & 0.0 & 0.0 & Class Gastropoda & & & \\
\hline Neanthes succinea & 13.0 & 5.0 & 3.0 & Crepidula unid. Spp. & 0.0 & 0.0 & 0.0 \\
\hline Polychaeta unid. spp. (1) & 0.0 & 0.0 & 0.0 & Gastropoda unid. sp. B & 0.0 & 0.0 & 0.0 \\
\hline Polychaeta unid. spp. (2) & 0.0 & 0.0 & 0.0 & $\begin{array}{l}\text { Gastropoda unia. sp. B } \\
\text { Ilyanassa obsoleta }\end{array}$ & 0.0 & 0.0 & 0.0 \\
\hline Polycirrus spp. & 0.0 & 0.0 & 0.0 & $\begin{array}{l}\text { lyyanassa obsoleta } \\
\text { Philine unid. spp. }\end{array}$ & 0.0 & 0.0 & 0.0 \\
\hline Polydora cornuta & 0.0 & 0.0 & 0.0 & $\begin{array}{l}\text { Philine unid. spp. } \\
\text { Urosalpinx cinerea }\end{array}$ & 0.0 & 0.0 & 0.0 \\
\hline Polydora unid. spp. & 0.0 & 0.0 & 0.0 & Orosaipinx cimerea & & 0.0 & 0.0 \\
\hline Pseudopolydora kempi & 0.0 & 0.0 & 0.0 & PHYLUIM NEMAIODA & & & \\
\hline Pseudopolydora paucibranchiata & 0.0 & 0.0 & 0.0 & Nematoda unid. spp. & 0.0 & 0.0 & 0.0 \\
\hline Sabaco elongatus & 0.0 & 0.0 & 0.0 & PHYLUM PLATYHELMINTHES & & & \\
\hline Sabellidae unid. spp. & 0.0 & 0.0 & 0.0 & Class Turbellaria & & & \\
\hline Sphaerosyllis californiensis & 0.0 & 0.0 & 0.0 & Turbellaria unid. sp. A & 0.0 & 0.0 & 0.0 \\
\hline Sphaerosyllis erinaceus & 0.0 & 0.0 & 0.0 & Turbellaria unid. spp. & 0.0 & 0.0 & 0.0 \\
\hline Sphaerosyllis unid. sp. A & 0.0 & 0.0 & 0.0 & & & & \\
\hline Streblospio benedicti & 1.0 & 7.0 & 0.0 & & & & \\
\hline Spionidae unid. spp. & 0.0 & 0.0 & 0.0 & & & & \\
\hline PHYLUM ARTHROPODA & & & & & & & \\
\hline Class Copepoda & & & & & & & \\
\hline Calanoida unid. spp. & 0.0 & 0.0 & 0.0 & & & & \\
\hline Harpacticoida unid. spp. & 0.0 & 0.0 & 0.0 & & & & \\
\hline Class Malacostraca & & & & & & & \\
\hline Order Amphipoda & & & & & & & \\
\hline Ampelisca abdita & 0.0 & 0.0 & 0.0 & & & & \\
\hline Ampithoe unid. spp. & 0.0 & 0.0 & 0.0 & & & & \\
\hline Caprella californica & 0.0 & 0.0 & 0.0 & & & & \\
\hline Corophium heteroceratum & 0.0 & 0.0 & 0.0 & & & & \\
\hline Corophiidae unid. spp. & 0.0 & 0.0 & 0.0 & & & & \\
\hline Grandidierella japonica & 0.0 & 11.0 & 122.0 & & & & \\
\hline Monocorophium acherusicum & 0.0 & 0.0 & 0.0 & & & & \\
\hline Monocorophium insidiosum & 0.0 & 0.0 & 0.0 & & & & \\
\hline Monocorophium unid. spp. & 0.0 & 156.0 & 1047.0 & & & & \\
\hline Oedicerotidae unid spp. & 0.0 & 0.0 & 0.0 & & & & \\
\hline Order Cumacea & & & & & & & \\
\hline Nippoleucon hinumensis & 0.0 & 0.0 & 0.0 & & & & \\
\hline Order Decapoda & & & & & & & \\
\hline Crangon franciscorum & 0.0 & 0.0 & 0.0 & & & & \\
\hline Hemigrapsus oregonensis & 0.0 & 0.0 & 0.0 & & & & \\
\hline Crab unid spp. & 0.0 & 0.0 & 0.0 & & & & \\
\hline Order Isopoda & & & & & & & \\
\hline Gnorisphaeroma oregonensis & 2.0 & 5.0 & 3.0 & & & & \\
\hline Paranthura japonica & 0.0 & 0.0 & 0.0 & & & & \\
\hline Synidotea laevidorsalis & 0.0 & 1.0 & 27.0 & & & & \\
\hline Synidotea unid. spp. & 0.0 & 0.0 & 0.0 & & & & \\
\hline Class Ostracoda & & & & & & & \\
\hline Eusarsiella zostericola & 0.0 & 0.0 & 0.0 & & & & \\
\hline Cyprideis unid. spp. & 0.0 & 0.0 & 0.0 & & & & \\
\hline Class Thecostraca & & & & & & & \\
\hline Balanomorpha unid. spp. & 0.0 & 0.0 & 0.0 & & & & \\
\hline Cirripedia unid. spp. & 0.0 & 0.0 & 0.0 & & & & \\
\hline
\end{tabular}


Appendix 11. CC1 Species List and Abundance-Continued

\begin{tabular}{|c|c|c|c|c|c|c|c|}
\hline TAXON & 25-Apr-14 & 24-Jun-14 & 19-Aug-14 & TAXON & 25-Apr-14 & 24-Jun-14 & 19-Aug-14 \\
\hline $\begin{array}{l}\text { PHYLUM ANNELIDA } \\
\text { Class Oligochaeta }\end{array}$ & & & & $\begin{array}{l}\text { PHYLUM BRYOZOA } \\
\text { Class Gymnolaemata }\end{array}$ & & & \\
\hline Naididae unid. spp. & 0.0 & 0.0 & 0.0 & Cheilostomatida unid. Spp. & 0.0 & 0.0 & 0.0 \\
\hline Oligochaeta unid. spp. & 0.0 & 0.0 & 0.0 & PHYLUM CHORDATA & & & \\
\hline Tubificidae unid. spp. & 0.0 & 0.0 & 0.0 & Class Ascidiacea & & & \\
\hline Class Polychaeta & & & & Molgula mahattensis & 0.0 & 0.0 & 0.0 \\
\hline Capitella capitata complex & 0.0 & 0.0 & 0.0 & PHYLUM CNIDARIA & & & \\
\hline Cirratulidae unid. spp. & 0.0 & 0.0 & 0.0 & Class Anthozoa & & & \\
\hline Eteone fauchaldi & 0.0 & 0.0 & 0.0 & Actiniaria - attached & 0.0 & 0.0 & 0.0 \\
\hline Eteone lighti & 0.0 & 0.0 & 0.0 & Actiniaria - burrowing & 0.0 & 0.0 & 0.0 \\
\hline Eteone unid. spp. & 0.0 & 0.0 & 0.0 & Actiniaria unid. spp. & 0.0 & 0.0 & 0.0 \\
\hline Euchone limnicola & 0.0 & 0.0 & 0.0 & Class Hydrozoa & & & \\
\hline Euchone unid. spp. & 0.0 & 0.0 & 0.0 & Hydrozoa unid spp. & 0.0 & 0.0 & 0.0 \\
\hline Exogone lourei & 0.0 & 0.0 & 0.0 & PHYLUM MOLLUSCA & & & \\
\hline Glycera unid. spp. & 0.0 & 0.0 & 0.0 & Class Bivalvia & & & \\
\hline Glycinde armigera & 0.0 & 0.0 & 0.0 & Bivalvia unid. spp. & 0.0 & 0.0 & 0.0 \\
\hline Glycinde picta & 0.0 & 0.0 & 0.0 & Gemma gemma & 1.0 & 0.0 & 0.0 \\
\hline Glycinde unid. sp. SF1 & 0.0 & 0.0 & 0.0 & Macoma petalum & 3.0 & 0.0 & 3.0 \\
\hline Glycinde unid. spp. & 0.0 & 0.0 & 0.0 & Musculista senhousia & 0.0 & 0.0 & 0.0 \\
\hline Harmothoe imbricata & 0.0 & 0.0 & 0.0 & Mya arenaria & 3.0 & 5.0 & 0.0 \\
\hline Heteromastus filiformis & 0.0 & 0.0 & 0.0 & Potamocorbula amurensis & $\begin{array}{l}3.0 \\
76.0\end{array}$ & 24.0 & 21.0 \\
\hline Leitoscoloplos pugettensis & 0.0 & 0.0 & 0.0 & Theora lubrica & 0.0 & $\begin{array}{c}24.0 \\
0.0\end{array}$ & $\begin{array}{c}21.0 \\
0.0\end{array}$ \\
\hline Maldanidae unid. spp. & 0.0 & 0.0 & 0.0 & Venerupis philippinarum & 0.0 & 0.0 & 0.0 \\
\hline Marphysa sanguinea & 0.0 & 0.0 & 0.0 & Class Gastropoda & & & \\
\hline Neanthes succinea & 5.0 & 11.0 & 6.0 & Crepidula unid. Spp. & 0.0 & 0.0 & 0.0 \\
\hline Polychaeta unid. spp. (1) & 0.0 & 0.0 & 0.0 & Gastropoda unid. sp. B & 0.0 & 0.0 & 0.0 \\
\hline Polychaeta unid. spp. (2) & 0.0 & 0.0 & 0.0 & Ilyanassa obsoleta & 0.0 & 0.0 & 0.0 \\
\hline Polycirrus spp. & 0.0 & 0.0 & 0.0 & $\begin{array}{l}\text { lyyanassa obsoleta } \\
\text { Philine unid. spp. }\end{array}$ & 0.0 & 0.0 & 0.0 \\
\hline Polydora cornuta & 0.0 & 0.0 & 0.0 & $\begin{array}{l}\text { Philine unid. spp. } \\
\text { Urosalpinx cinerea }\end{array}$ & 0.0 & 0.0 & 0.0 \\
\hline Polydora unid. spp. & 0.0 & 0.0 & 0.0 & PHYLUM NEMATODA & & & \\
\hline Pseudopolydora kempi & 0.0 & 0.0 & 0.0 & & 0.0 & 0.0 & 0.0 \\
\hline Pseudopolydora paucibranchiata & 0.0 & 0.0 & 0.0 & PHYLUM PLATYHELMINTHES & & & \\
\hline Sabaco elongatus & 0.0 & 0.0 & 0.0 & $\begin{array}{l}\text { PHYLUM PLATYHELIMINTHES } \\
\text { Class Turbellaria }\end{array}$ & & & \\
\hline Sabellidae unid. spp. & 0.0 & 0.0 & 0.0 & Turbellaria unid. sp. A & 0.0 & 0.0 & 0.0 \\
\hline $\begin{array}{l}\text { Sphaerosyllis californiensis } \\
\text { Sphaerosyllis erinaceus }\end{array}$ & $\begin{array}{l}0.0 \\
0.0\end{array}$ & $\begin{array}{l}0.0 \\
0.0\end{array}$ & $\begin{array}{l}0.0 \\
0.0\end{array}$ & $\begin{array}{l}\text { Iurbellarıa unid. sp. A } \\
\text { Turbellaria unid. spp. }\end{array}$ & 0.0 & 0.0 & 0.0 \\
\hline Sphaerosyllis unid. sp. A & 0.0 & 0.0 & 0.0 & & & & \\
\hline Streblospio benedicti & 0.0 & 0.0 & 0.0 & & & & \\
\hline Spionidae unid. spp. & 0.0 & 0.0 & 0.0 & & & & \\
\hline \multicolumn{8}{|l|}{ PHYLUM ARTHROPODA } \\
\hline \multicolumn{8}{|l|}{ Class Copepoda } \\
\hline Calanoida unid. spp. & 0.0 & 0.0 & 0.0 & & & & \\
\hline Harpacticoida unid. spp. & 0.0 & 0.0 & 0.0 & & & & \\
\hline \multicolumn{8}{|l|}{ Class Malacostraca } \\
\hline \multicolumn{8}{|l|}{ Order Amphipoda } \\
\hline Ampelisca abdita & 0.0 & 0.0 & 0.0 & & & & \\
\hline Ampithoe unid. spp. & 0.0 & 0.0 & 0.0 & & & & \\
\hline Caprella californica & 0.0 & 0.0 & 0.0 & & & & \\
\hline Corophium heteroceratum & 0.0 & 0.0 & 0.0 & & & & \\
\hline Corophiidae unid. spp. & 0.0 & 0.0 & 0.0 & & & & \\
\hline Grandidierella japonica & 1.0 & 1.0 & 3.0 & & & & \\
\hline Monocorophium acherusicum & 0.0 & 0.0 & 0.0 & & & & \\
\hline Monocorophium insidiosum & 0.0 & 0.0 & 0.0 & & & & \\
\hline Monocorophium unid. spp. & 3576.0 & 133.0 & 28.0 & & & & \\
\hline Oedicerotidae unid spp. & 0.0 & 0.0 & 0.0 & & & & \\
\hline \multicolumn{8}{|l|}{ Order Cumacea } \\
\hline Nippoleucon hinumensis & 0.0 & 0.0 & 0.0 & & & & \\
\hline \multicolumn{8}{|l|}{ Order Decapoda } \\
\hline Crangon franciscorum & 0.0 & 0.0 & 0.0 & & & & \\
\hline Hemigrapsus oregonensis & 0.0 & 0.0 & 0.0 & & & & \\
\hline Crab unid spp. & 0.0 & 0.0 & 0.0 & & & & \\
\hline \multicolumn{8}{|l|}{ Order Isopoda } \\
\hline Gnorisphaeroma oregonensis & 16.0 & 1.0 & 0.0 & & & & \\
\hline Paranthura japonica & 0.0 & 0.0 & 0.0 & & & & \\
\hline Synidotea laevidorsalis & 37.0 & 8.0 & 0.0 & & & & \\
\hline Synidotea unid. spp. & 0.0 & 0.0 & 0.0 & & & & \\
\hline \multicolumn{8}{|l|}{ Class Ostracoda } \\
\hline Eusarsiella zostericola & 0.0 & 0.0 & 0.0 & & & & \\
\hline \multirow{2}{*}{\multicolumn{8}{|c|}{ Class Thecostraca }} \\
\hline & & & & & & & \\
\hline Balanomorpha unid. spp. & 0.0 & 0.0 & 0.0 & & & & \\
\hline Cirripedia unid. spp. & 0.0 & 0.0 & 0.0 & & & & \\
\hline
\end{tabular}


Appendix 11. CC1 Species List and Abundance-Continued

\begin{tabular}{|c|c|c|c|}
\hline TAXON & 24-Sep-14 & 21-Oct-14 & 13-Nov-14 \\
\hline \multicolumn{4}{|l|}{ PHYLUM ANNELIDA } \\
\hline \multicolumn{4}{|l|}{ Class Oligochaeta } \\
\hline Naididae unid. spp. & 0.0 & 0.0 & 0.0 \\
\hline Oligochaeta unid. spp. & 0.0 & 0.0 & 0.0 \\
\hline Tubificidae unid. spp. & 0.0 & 0.0 & 0.0 \\
\hline \multicolumn{4}{|l|}{ Class Polychaeta } \\
\hline Capitella capitata complex & 0.0 & 0.0 & 0.0 \\
\hline Cirratulidae unid. spp. & 0.0 & 0.0 & 0.0 \\
\hline Eteone fauchaldi & 0.0 & 0.0 & 0.0 \\
\hline Eteone lighti & 0.0 & 0.0 & 0.0 \\
\hline Eteone unid. spp. & 0.0 & 0.0 & 0.0 \\
\hline Euchone limnicola & 0.0 & 0.0 & 0.0 \\
\hline Euchone unid. spp. & 0.0 & 0.0 & 0.0 \\
\hline Exogone lourei & 0.0 & 0.0 & 0.0 \\
\hline Glycera unid. spp. & 0.0 & 0.0 & 0.0 \\
\hline Glycinde armigera & 0.0 & 0.0 & 0.0 \\
\hline Glycinde picta & 0.0 & 0.0 & 0.0 \\
\hline Glycinde unid. sp. SF1 & 0.0 & 0.0 & 0.0 \\
\hline Glycinde unid. spp. & 0.0 & 0.0 & 0.0 \\
\hline Harmothoe imbricata & 0.0 & 0.0 & 0.0 \\
\hline Heteromastus filiformis & 0.0 & 1.0 & 0.0 \\
\hline Leitoscoloplos pugettensis & 0.0 & 0.0 & 0.0 \\
\hline Maldanidae unid. spp. & 0.0 & 0.0 & 0.0 \\
\hline Marphysa sanguinea & 0.0 & 0.0 & 0.0 \\
\hline Neanthes succinea & 8.0 & 11.0 & 0.0 \\
\hline Polychaeta unid. spp. (1) & 0.0 & 0.0 & 0.0 \\
\hline Polychaeta unid. spp. (2) & 0.0 & 0.0 & 0.0 \\
\hline Polycirrus spp. & 0.0 & 0.0 & 0.0 \\
\hline Polydora cornuta & 0.0 & 0.0 & 0.0 \\
\hline Polydora unid. spp. & 0.0 & 0.0 & 0.0 \\
\hline Pseudopolydora kempi & 0.0 & 0.0 & 0.0 \\
\hline Pseudopolydora paucibranchiata & 0.0 & 0.0 & 0.0 \\
\hline Sabaco elongatus & 0.0 & 0.0 & 0.0 \\
\hline Sabellidae unid. spp. & 0.0 & 0.0 & 0.0 \\
\hline Sphaerosyllis californiensis & 0.0 & 0.0 & 0.0 \\
\hline Sphaerosyllis erinaceus & 0.0 & 0.0 & 0.0 \\
\hline Sphaerosyllis unid. sp. A & 0.0 & 0.0 & 0.0 \\
\hline Streblospio benedicti & 0.0 & 0.0 & 0.0 \\
\hline Spionidae unid. spp. & 0.0 & 0.0 & 0.0 \\
\hline \multicolumn{4}{|l|}{ PHYLUM ARTHROPODA } \\
\hline \multicolumn{4}{|l|}{ Class Copepoda } \\
\hline Calanoida unid. spp. & 0.0 & 0.0 & 0.0 \\
\hline Harpacticoida unid. spp. & 0.0 & 0.0 & 0.0 \\
\hline \multicolumn{4}{|l|}{ Class Malacostraca } \\
\hline \multicolumn{4}{|l|}{ Order Amphipoda } \\
\hline Ampelisca abdita & 0.0 & 0.0 & 0.0 \\
\hline Ampithoe unid. spp. & 0.0 & 0.0 & 0.0 \\
\hline Caprella californica & 0.0 & 0.0 & 0.0 \\
\hline Corophium heteroceratum & 0.0 & 0.0 & 0.0 \\
\hline Corophiidae unid. spp. & 0.0 & 0.0 & 0.0 \\
\hline Grandidierella japonica & 18.0 & 28.0 & 0.0 \\
\hline Monocorophium acherusicum & 1.0 & 66.0 & 0.0 \\
\hline Monocorophium insidiosum & 0.0 & 0.0 & 0.0 \\
\hline Monocorophium unid. spp. & 215.0 & 4627.0 & 2217.0 \\
\hline Oedicerotidae unid spp. & 0.0 & 0.0 & 0.0 \\
\hline \multicolumn{4}{|l|}{ Order Cumacea } \\
\hline Nippoleucon hinumensis & 0.0 & 0.0 & 0.0 \\
\hline \multicolumn{4}{|l|}{ Order Decapoda } \\
\hline Crangon franciscorum & 0.0 & 0.0 & 0.0 \\
\hline Hemigrapsus oregonensis & 0.0 & 0.0 & 0.0 \\
\hline Crab unid spp. & 0.0 & 0.0 & 0.0 \\
\hline \multicolumn{4}{|l|}{ Order Isopoda } \\
\hline Gnorisphaeroma oregonensis & 0.0 & 0.0 & 0.0 \\
\hline Paranthura japonica & 0.0 & 1.0 & 0.0 \\
\hline Synidotea laevidorsalis & 3.0 & 16.0 & 0.0 \\
\hline Synidotea unid. spp. & 0.0 & 1.0 & 0.0 \\
\hline \multicolumn{4}{|l|}{ Class Ostracoda } \\
\hline Eusarsiella zostericola & 0.0 & 0.0 & 0.0 \\
\hline Cyprideis unid. spp. & 0.0 & 0.0 & 0.0 \\
\hline Class Thecostraca & & & \\
\hline Balanomorpha unid. spp. & 0.0 & 0.0 & 0.0 \\
\hline Cirripedia unid. spp. & 0.0 & 0.0 & 0.0 \\
\hline
\end{tabular}

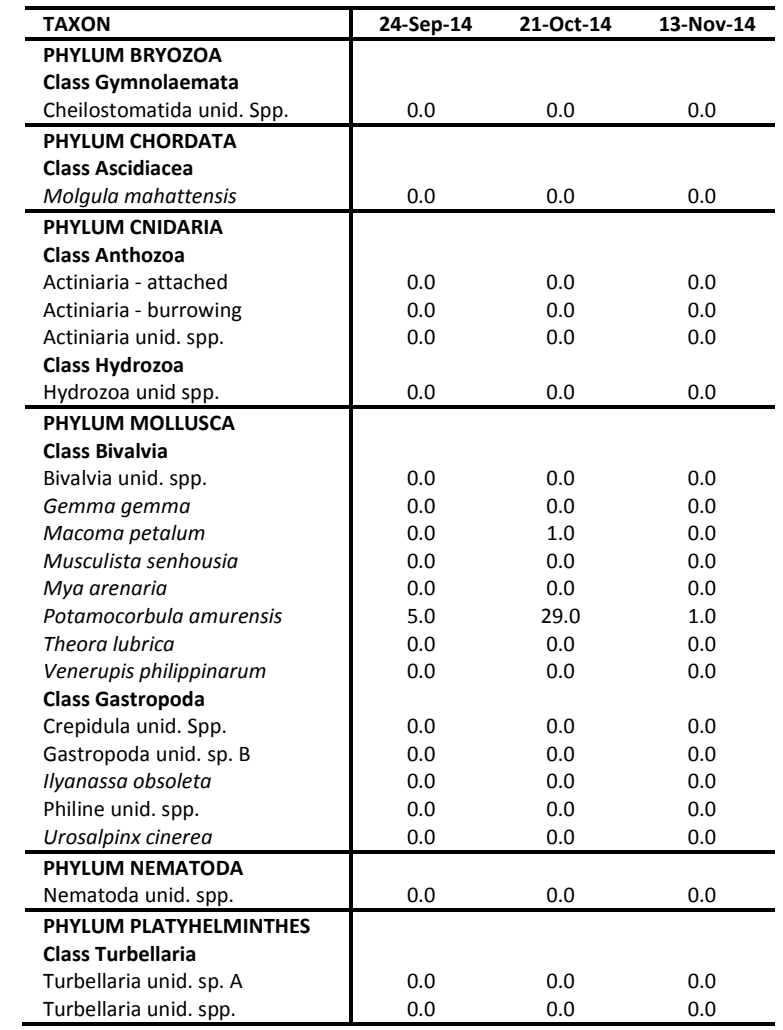


Appendix 12. D3 Species List and Abundance

\begin{tabular}{|c|c|c|c|c|c|c|c|}
\hline TAXON & 28-Jan-1 & 27-Feb-1 & 27-Mar-1 & TAXON & 28-Jan-14 & 27-Feb-14 & 27-Mar-14 \\
\hline $\begin{array}{l}\text { PHYLUM ANNELIDA } \\
\text { Class Oligochaeta } \\
\text { Naididae unid. spp. }\end{array}$ & 0.0 & 0.0 & 0.0 & $\begin{array}{l}\text { PHYLUM BRYOZOA } \\
\text { Class Gymnolaemata } \\
\text { Cheilostomatida unid. Spp. }\end{array}$ & 0.0 & 0.0 & 0.0 \\
\hline Oligochaeta unid. spp. & 0.0 & 0.0 & 0.0 & PHYLUM CHORDATA & & & \\
\hline Tubificidae unid. spp. & 0.0 & 0.0 & 0.0 & Class Ascidiacea & & & \\
\hline Class Polychaeta & & & & Molgula mahattensis & 0.0 & 0.0 & 0.0 \\
\hline Capitella capitata complex & 0.0 & 0.0 & 0.0 & PHYLUM CNIDARIA & & & \\
\hline Cirratulidae unid. spp. & 0.0 & 0.0 & 0.0 & Class Anthozoa & & & \\
\hline Eteone fauchaldi & 0.0 & 0.0 & 0.0 & Actiniaria - attached & 3.0 & 0.0 & 0.0 \\
\hline Eteone lighti & 3.0 & 0.0 & 0.0 & Actiniaria - burrowing & 0.0 & 0.0 & 0.0 \\
\hline Eteone unid. spp. & 0.0 & 0.0 & 0.0 & Actiniaria unid. spp. & 0.0 & 0.0 & 0.0 \\
\hline Euchone limnicola & 0.0 & 0.0 & 0.0 & Class Hydrozoa & & & \\
\hline Euchone unid. spp. & 0.0 & 0.0 & 0.0 & Hydrozoa unid spp. & 0.0 & 0.0 & 0.0 \\
\hline Exogone lourei & 0.0 & 0.0 & 0.0 & PHYLUM MOLLUSCA & & & \\
\hline Glycera unid. spp. & 0.0 & 0.0 & 0.0 & Class Bivalvia & & & \\
\hline Glycinde armigera & 0.0 & 0.0 & 0.0 & Bivalvia unid. spp. & 0.0 & 0.0 & 0.0 \\
\hline Glycinde picta & 0.0 & 1.0 & 0.0 & Gemma gemma & 157.0 & 0.0 & 94.0 \\
\hline Glycinde unid. sp. SF1 & 0.0 & 0.0 & 0.0 & Macoma petalum & 16.0 & 0.0 & 3.0 \\
\hline Glycinde unid. spp. & 0.0 & 0.0 & 0.0 & Musculista senhousia & 0.0 & 0.0 & 0.0 \\
\hline Harmothoe imbricata & 0.0 & 0.0 & 0.0 & Mya arenaria & 1.0 & 1.0 & 0.0 \\
\hline Heteromastus filiformis & 10.0 & 0.0 & 0.0 & $\begin{array}{l}\text { Plya arenaria } \\
\text { Potamocorbula amurensis }\end{array}$ & $\begin{array}{l}1.0 \\
9.0\end{array}$ & $\begin{array}{l}1.0 \\
0.0\end{array}$ & 0.0 \\
\hline Leitoscoloplos pugettensis & 0.0 & 0.0 & 0.0 & $\begin{array}{l}\text { Theoramocorbula amurensis } \\
\text { Theorica }\end{array}$ & 0.0 & $\begin{array}{l}0.0 \\
1.0\end{array}$ & 1.0 \\
\hline Maldanidae unid. spp. & 0.0 & 0.0 & 0.0 & Venerupis philippinarum & 0.0 & 0.0 & 0.0 \\
\hline Marphysa sanguinea & 0.0 & 0.0 & 0.0 & Class Gastropoda & & & \\
\hline Neanthes succinea & 18.0 & 3.0 & 1.0 & Crepidula unid. Spp. & 0.0 & 0.0 & 0.0 \\
\hline Polychaeta unid. spp. (1) & 0.0 & 0.0 & 0.0 & Gastropoda unid. sp. B & 0.0 & 0.0 & 0.0 \\
\hline Polychaeta unid. spp. (2) & 0.0 & 0.0 & 0.0 & Ilyanassa obsoleta & 0.0 & 0.0 & 1.0 \\
\hline Polycirrus spp. & 0.0 & 0.0 & 0.0 & $\begin{array}{l}\text { Philine unid. spp. } \\
\text { Ppota }\end{array}$ & 0.0 & 0.0 & $\begin{array}{l}1.0 \\
0.0\end{array}$ \\
\hline Polydora cornuta & 0.0 & 0.0 & 0.0 & Urosalpinx cinerea & 0.0 & 0.0 & 0.0 \\
\hline Polydora unid. spp. & 0.0 & 0.0 & 0.0 & PHYLUM NEMATODA & & & \\
\hline Pseudopolydora kempi & 0.0 & 0.0 & 0.0 & Nematoda unid. spp. & 0.0 & 0.0 & 0.0 \\
\hline Pseudopolydora paucibranchiata & 0.0 & 0.0 & 0.0 & PHYLUM PLATYHELMINTHES & & & \\
\hline Sabaco elongatus & 0.0 & 0.0 & 0.0 & Class Turbellaria & & & \\
\hline Sabellidae unid. spp. & 0.0 & 0.0 & 0.0 & $\begin{array}{l}\text { Class Turbellaria } \\
\text { Turbellaria unid. sp. A }\end{array}$ & & 0.0 & 00 \\
\hline Sphaerosyllis californiensis & 0.0 & 0.0 & 0.0 & & 0.0 & 0.0 & 0.0 \\
\hline Sphaerosyllis erinaceus & 0.0 & 0.0 & 0.0 & Turbellaria unid. spp. & 0.0 & 0.0 & 0.0 \\
\hline Sphaerosyllis unid. sp. A & 0.0 & 0.0 & 0.0 & & & & \\
\hline Streblospio benedicti & 19.0 & 0.0 & 0.0 & & & & \\
\hline Spionidae unid. spp. & 0.0 & 0.0 & 0.0 & & & & \\
\hline PHYLUM ARTHROPODA & & & & & & & \\
\hline Class Copepoda & & & & & & & \\
\hline Calanoida unid. spp. & 0.0 & 0.0 & 0.0 & & & & \\
\hline Harpacticoida unid. spp. & 0.0 & 0.0 & 0.0 & & & & \\
\hline Class Malacostraca & & & & & & & \\
\hline Order Amphipoda & & & & & & & \\
\hline Ampelisca abdita & 81.0 & 219.0 & 1.0 & & & & \\
\hline Ampithoe unid. spp. & 0.0 & 0.0 & 0.0 & & & & \\
\hline Caprella californica & 0.0 & 0.0 & 0.0 & & & & \\
\hline Corophium heteroceratum & 0.0 & 19.0 & 1.0 & & & & \\
\hline Corophiidae unid. spp. & 0.0 & 0.0 & 0.0 & & & & \\
\hline Grandidierella japonica & 3.0 & 0.0 & 0.0 & & & & \\
\hline Monocorophium acherusicum & 0.0 & 0.0 & 0.0 & & & & \\
\hline Monocorophium insidiosum & 0.0 & 0.0 & 0.0 & & & & \\
\hline Monocorophium unid. spp. & 36.0 & 0.0 & 0.0 & & & & \\
\hline Oedicerotidae unid spp. & 0.0 & 0.0 & 0.0 & & & & \\
\hline Order Cumacea & & & & & & & \\
\hline Nippoleucon hinumensis & 0.0 & 1.0 & 67.0 & & & & \\
\hline Order Decapoda & & & & & & & \\
\hline Crangon franciscorum & 0.0 & 0.0 & 0.0 & & & & \\
\hline Hemigrapsus oregonensis & 0.0 & 0.0 & 0.0 & & & & \\
\hline Crab unid spp. & 0.0 & 0.0 & 0.0 & & & & \\
\hline Order Isopoda & & & & & & & \\
\hline Gnorisphaeroma oregonensis & 0.0 & 0.0 & 0.0 & & & & \\
\hline Paranthura japonica & 0.0 & 0.0 & 0.0 & & & & \\
\hline Synidotea laevidorsalis & 0.0 & 1.0 & 0.0 & & & & \\
\hline Synidotea unid. spp. & 0.0 & 0.0 & 0.0 & & & & \\
\hline Class Ostracoda & & & & & & & \\
\hline Eusarsiella zostericola & 0.0 & 0.0 & 0.0 & & & & \\
\hline Cyprideis unid. spp. & 0.0 & 0.0 & 0.0 & & & & \\
\hline Class Thecostraca & & & & & & & \\
\hline Balanomorpha unid. spp. & 0.0 & 0.0 & 0.0 & & & & \\
\hline Cirripedia unid. spp. & 0.0 & 0.0 & 0.0 & & & & \\
\hline
\end{tabular}




\section{Appendix 12. D3 Species List and Abundance-Continued}

\begin{tabular}{|c|c|c|c|c|c|c|c|}
\hline TAXON & 25-Apr-14 & 24-Jun-14 & 19-Aug-14 & TAXON & 25-Apr-14 & 24-Jun-14 & 19-Aug-14 \\
\hline $\begin{array}{l}\text { PHYLUM ANNELIDA } \\
\text { Class Oligochaeta } \\
\text { Naididae unid. spp. }\end{array}$ & 0.0 & 0.0 & 0.0 & $\begin{array}{l}\text { PHYLUM BRYOZOA } \\
\text { Class Gymnolaemata } \\
\text { Cheilostomatida unid. Spp. }\end{array}$ & 0.0 & 0.0 & 0.0 \\
\hline Oligochaeta unid. spp. & 0.0 & 0.0 & 0.0 & PHYLUM CHORDATA & & & \\
\hline Tubificidae unid. spp. & 0.0 & 0.0 & 0.0 & Class Ascidiacea & & & \\
\hline Class Polychaeta & & & & Molgula mahattensis & 0.0 & 0.0 & 0.0 \\
\hline Capitella capitata complex & 0.0 & 0.0 & 0.0 & PHYLUM CNIDARIA & & & \\
\hline Cirratulidae unid. spp. & 0.0 & 0.0 & 0.0 & Class Anthozoa & & & \\
\hline Eteone fauchaldi & 0.0 & 0.0 & 0.0 & Actiniaria - attached & 4.0 & 2.0 & 4.0 \\
\hline Eteone lighti & 0.0 & 0.0 & 0.0 & Actiniaria - burrowing & 0.0 & 0.0 & 0.0 \\
\hline Eteone unid. spp. & 0.0 & 0.0 & 0.0 & Actiniaria unid. spp. & 0.0 & 0.0 & 0.0 \\
\hline Euchone limnicola & 0.0 & 0.0 & 0.0 & Class Hydrozoa & & & \\
\hline Euchone unid. spp. & 0.0 & 0.0 & 0.0 & Hydrozoa unid spp. & 0.0 & 0.0 & 0.0 \\
\hline Exogone lourei & 0.0 & 0.0 & 0.0 & PHYLUM MOLLUSCA & & & \\
\hline Glycera unid. spp. & 0.0 & 0.0 & 0.0 & Class Bivalvia & & & \\
\hline Glycinde armigera & 0.0 & 0.0 & 0.0 & Bivalvia unid. spp. & 0.0 & 0.0 & 0.0 \\
\hline Glycinde picta & 0.0 & 1.0 & 0.0 & Gemma gemma & 1068.0 & 1377.0 & 0.0 \\
\hline Glycinde unid. sp. SF1 & 0.0 & 0.0 & 0.0 & Macoma petalum & 26.0 & 11.0 & 8.0 \\
\hline Glycinde unid. spp. & 0.0 & 0.0 & 0.0 & Musculista senhousia & $\begin{array}{l}20.0 \\
0.0\end{array}$ & 0.0 & $\begin{array}{l}0.0 \\
0.0\end{array}$ \\
\hline Harmothoe imbricata & 0.0 & 0.0 & 0.0 & Mya arenaria & 5.0 & $\begin{array}{l}0.0 \\
1.0\end{array}$ & 3.0 \\
\hline Heteromastus filiformis & 1.0 & 2.0 & 18.0 & Potamocorbula amurensis & 11.0 & 25.0 & 10.0 \\
\hline Leitoscoloplos pugettensis & 0.0 & 0.0 & 0.0 & Theora lubrica & 0.0 & 1.0 & 0.0 \\
\hline Maldanidae unid. spp. & 0.0 & 0.0 & 0.0 & Venerupis philippinarum & 1.0 & 0.0 & 0.0 \\
\hline Marphysa sanguinea & 0.0 & 0.0 & 0.0 & Class Gastropoda & 1.0 & 0.0 & 0.0 \\
\hline Neanthes succinea & 29.0 & 5.0 & 13.0 & Crepidula unid. Spp. & 0.0 & 0.0 & 0.0 \\
\hline Polychaeta unid. spp. (1) & 0.0 & 0.0 & 0.0 & Gastropoda unid. sp. B & 0.0 & 0.0 & 0.0 \\
\hline Polychaeta unid. spp. (2) & 0.0 & 0.0 & 0.0 & Ilyanassa obsoleta & 0.0 & 0.0 & 0.0 \\
\hline Polycirrus spp. & 0.0 & 0.0 & 0.0 & Philine unid. spp. & 0.0 & 0.0 & 0.0 \\
\hline Polydora cornuta & 0.0 & 0.0 & 0.0 & Urosalpinx cinerea & 0.0 & 0.0 & 0.0 \\
\hline Polydora unid. spp. & 0.0 & 0.0 & 0.0 & PHYLUM NEMATODA & & & \\
\hline Pseudopolydora kempi & 0.0 & 0.0 & 0.0 & Nematoda unid. spp. & 0.0 & 0.0 & 0.0 \\
\hline $\begin{array}{l}\text { Pseudopolydora paucibranchiata } \\
\text { Sabaco elongatus }\end{array}$ & $\begin{array}{l}0.0 \\
0.0\end{array}$ & $\begin{array}{l}0.0 \\
0.0\end{array}$ & $\begin{array}{l}0.0 \\
0.0\end{array}$ & PHYLUM PLATYHELMINTHES & & & \\
\hline Sabellidae unid. spp. & 0.0 & 0.0 & 0.0 & Class Turbellaria & & & \\
\hline Sphaerosyllis californiensis & 0.0 & 0.0 & 0.0 & Turbellaria unid. sp. A & 0.0 & 0.0 & 0.0 \\
\hline Sphaerosyllis erinaceus & 0.0 & 0.0 & 0.0 & Turbellaria unid. spp. & 0.0 & 0.0 & 0.0 \\
\hline Sphaerosyllis unid. sp. A & 0.0 & 0.0 & 0.0 & & & & \\
\hline Streblospio benedicti & 0.0 & 0.0 & 0.0 & & & & \\
\hline Spionidae unid. spp. & 0.0 & 0.0 & 0.0 & & & & \\
\hline PHYLUM ARTHROPODA & & & & & & & \\
\hline Class Copepoda & & & & & & & \\
\hline Calanoida unid. spp. & 0.0 & 0.0 & 0.0 & & & & \\
\hline Harpacticoida unid. spp. & 0.0 & 0.0 & 0.0 & & & & \\
\hline Class Malacostraca & & & & & & & \\
\hline Order Amphipoda & & & & & & & \\
\hline Ampelisca abdita & 15.0 & 73.0 & 266.0 & & & & \\
\hline Ampithoe unid. spp. & 0.0 & 0.0 & 0.0 & & & & \\
\hline Caprella californica & 0.0 & 0.0 & 0.0 & & & & \\
\hline Corophium heteroceratum & 10.0 & 2.0 & 0.0 & & & & \\
\hline Corophiidae unid. spp. & 0.0 & 0.0 & 0.0 & & & & \\
\hline Grandidierella japonica & 0.0 & 0.0 & 2.0 & & & & \\
\hline Monocorophium acherusicum & 0.0 & 0.0 & 0.0 & & & & \\
\hline Monocorophium insidiosum & 0.0 & 0.0 & 0.0 & & & & \\
\hline Monocorophium unid. spp. & 0.0 & 0.0 & 0.0 & & & & \\
\hline Oedicerotidae unid spp. & 0.0 & 0.0 & 0.0 & & & & \\
\hline Order Cumacea & & & & & & & \\
\hline Nippoleucon hinumensis & 0.0 & 0.0 & 0.0 & & & & \\
\hline Order Decapoda & & & & & & & \\
\hline Crangon franciscorum & 0.0 & 0.0 & 0.0 & & & & \\
\hline Hemigrapsus oregonensis & 0.0 & 0.0 & 0.0 & & & & \\
\hline Crab unid spp. & 0.0 & 0.0 & 0.0 & & & & \\
\hline Order Isopoda & & & & & & & \\
\hline Gnorisphaeroma oregonensis & 0.0 & 0.0 & 0.0 & & & & \\
\hline Paranthura japonica & 0.0 & 0.0 & 0.0 & & & & \\
\hline Synidotea laevidorsalis & 3.0 & 3.0 & 0.0 & & & & \\
\hline Synidotea unid. spp. & 0.0 & 0.0 & 0.0 & & & & \\
\hline Class Ostracoda & & & & & & & \\
\hline Eusarsiella zostericola & 0.0 & 0.0 & 0.0 & & & & \\
\hline Cyprideis unid. spp. & 0.0 & 0.0 & 0.0 & & & & \\
\hline Class Thecostraca & & & & & & & \\
\hline Balanomorpha unid. spp. & 0.0 & 0.0 & 0.0 & & & & \\
\hline Cirripedia unid. spp. & 0.0 & 0.0 & 0.0 & & & & \\
\hline
\end{tabular}




\section{Appendix 12. D3 Species List and Abundance-Continued}

\begin{tabular}{|c|c|c|c|c|c|c|c|}
\hline TAXON & 24-Sep-14 & 21-Oct-14 & 13-Nov-14 & TAXON & 24-Sep-14 & 21-Oct-14 & 13-Nov-14 \\
\hline $\begin{array}{l}\text { PHYLUM ANNELIDA } \\
\text { Class Oligochaeta }\end{array}$ & & & & $\begin{array}{l}\text { PHYLUM BRYOZOA } \\
\text { Class Gymnolaemata }\end{array}$ & & & \\
\hline Naididae unid. spp. & 0.0 & 0.0 & 0.0 & Cheilostomatida unid. Spp. & 0.0 & 0.0 & 0.0 \\
\hline Oligochaeta unid. spp. & 0.0 & 0.0 & 0.0 & PHYLUM CHORDATA & & & \\
\hline Tubificidae unid. spp. & 0.0 & 0.0 & 0.0 & Class Ascidiacea & & & \\
\hline Class Polychaeta & & & & Molgula mahattensis & 0.0 & 0.0 & 0.0 \\
\hline Capitella capitata complex & 0.0 & 0.0 & 0.0 & PHYLUM CNIDARIA & & & \\
\hline Cirratulidae unid. spp. & 0.0 & 0.0 & 0.0 & Class Anthozoa & & & \\
\hline Eteone fauchaldi & 0.0 & 0.0 & 0.0 & Actiniaria - attached & 2.0 & 0.0 & 0.0 \\
\hline Eteone lighti & 0.0 & 0.0 & 0.0 & Actiniaria - burrowing & 0.0 & 0.0 & 0.0 \\
\hline Eteone unid. spp. & 0.0 & 0.0 & 0.0 & Actiniaria unid. spp. & 0.0 & 0.0 & 0.0 \\
\hline Euchone limnicola & 0.0 & 0.0 & 0.0 & Class Hydrozoa & & & \\
\hline Euchone unid. spp. & 0.0 & 0.0 & 0.0 & Hydrozoa unid spp. & 0.0 & 0.0 & 0.0 \\
\hline Exogone lourei & 0.0 & 0.0 & 0.0 & PHYLUM MOLLUSCA & & & \\
\hline Glycera unid. spp. & 0.0 & 0.0 & 0.0 & Class Bivalvia & & & \\
\hline Glycinde armigera & 0.0 & 0.0 & 0.0 & Bivalvia unid. spp. & 0.0 & 0.0 & 0.0 \\
\hline Glycinde picta & 0.0 & 0.0 & 0.0 & Gemma gemma & 49.0 & 20.0 & 0.0 \\
\hline Glycinde unid. sp. SF1 & 0.0 & 0.0 & 0.0 & Macoma petalum & 1.0 & 6.0 & 16.0 \\
\hline Glycinde unid. spp. & 0.0 & 0.0 & 0.0 & Musculista senhousia & 0.0 & 0.0 & 0.0 \\
\hline Harmothoe imbricata & 0.0 & 0.0 & 0.0 & Mya arenaria & 0.0 & 0.0 & 0.0 \\
\hline Heteromastus filiformis & 4.0 & 10.0 & 0.0 & Potamocorbula amurensis & 7.0 & 14.0 & 2.0 \\
\hline Leitoscoloplos pugettensis & 0.0 & 0.0 & 0.0 & Theora lubrica & 1.0 & 0.0 & 0.0 \\
\hline Maldanidae unid. spp. & 0.0 & 0.0 & 0.0 & Venerupis philippinarum & 0.0 & 0.0 & 0.0 \\
\hline Marphysa sanguinea & 0.0 & 0.0 & 0.0 & Class Gastropoda & 0.0 & 0.0 & \\
\hline Neanthes succinea & 7.0 & 0.0 & 0.0 & Crepidula unid. Spp. & 0.0 & 0.0 & 0.0 \\
\hline Polychaeta unid. spp. (1) & 0.0 & 0.0 & 0.0 & Gastropoda unid. sp. B & 0.0 & 0.0 & 0.0 \\
\hline Polychaeta unid. spp. (2) & 0.0 & 0.0 & 0.0 & $\begin{array}{l}\text { Ilyanassa obsoleta } \\
\text { lla }\end{array}$ & 0.0 & 1.0 & 14.0 \\
\hline Polycirrus spp. & 0.0 & 0.0 & 0.0 & Philine unid. spp. & 0.0 & 0.0 & 0.0 \\
\hline Polydora cornuta & 0.0 & 0.0 & 0.0 & Urosalpinx cinerea & 0.0 & 0.0 & 0.0 \\
\hline Polydora unid. spp. & 0.0 & 0.0 & 0.0 & PHYLUM NEMATODA & & & \\
\hline Pseudopolydora kempi & 0.0 & 0.0 & 0.0 & Nematoda unid. spp. & 0.0 & 0.0 & 0.0 \\
\hline Pseudopolydora paucibranchiata & 0.0 & $\begin{array}{l}0.0 \\
0.0\end{array}$ & $\begin{array}{l}0.0 \\
0.0\end{array}$ & PHYLUM PLATYHELMINTHES & & & \\
\hline $\begin{array}{l}\text { Sabaco elongatus } \\
\text { Sabellidae unid. spp. }\end{array}$ & $\begin{array}{l}0.0 \\
0.0\end{array}$ & $\begin{array}{l}0.0 \\
0.0\end{array}$ & $\begin{array}{l}0.0 \\
0.0\end{array}$ & Class Turbellaria & & & \\
\hline $\begin{array}{l}\text { Sabellidae unid. spp. } \\
\text { Sphaerosyllis californiensis }\end{array}$ & 0.0 & $\begin{array}{l}0.0 \\
0.0\end{array}$ & 0.0 & Turbellaria unid. sp. A & 0.0 & 0.0 & 0.0 \\
\hline $\begin{array}{l}\text { Sphaerosyllis californiensis } \\
\text { Sphaerosyllis erinaceus }\end{array}$ & 0.0 & 0.0 & 0.0 & Turbellaria unid. spp. & 0.0 & 0.0 & 0.0 \\
\hline Sphaerosyllis unid. sp. A & 0.0 & 0.0 & 0.0 & & & & \\
\hline Streblospio benedicti & 0.0 & 0.0 & 0.0 & & & & \\
\hline Spionidae unid. spp. & 0.0 & 0.0 & 0.0 & & & & \\
\hline PHYLUM ARTHROPODA & & & & & & & \\
\hline Class Copepoda & & & & & & & \\
\hline Calanoida unid. spp. & 0.0 & 0.0 & 0.0 & & & & \\
\hline Harpacticoida unid. spp. & 0.0 & 0.0 & 0.0 & & & & \\
\hline Class Malacostraca & & & & & & & \\
\hline Order Amphipoda & & & & & & & \\
\hline Ampelisca abdita & 301.0 & 0.0 & 15.0 & & & & \\
\hline Ampithoe unid. spp. & 0.0 & 0.0 & 0.0 & & & & \\
\hline Caprella californica & 0.0 & 0.0 & 0.0 & & & & \\
\hline Corophium heteroceratum & 0.0 & 2.0 & 0.0 & & & & \\
\hline Corophiidae unid. spp. & 0.0 & 0.0 & 0.0 & & & & \\
\hline Grandidierella japonica & 1.0 & 2.0 & 0.0 & & & & \\
\hline Monocorophium acherusicum & 0.0 & 0.0 & 0.0 & & & & \\
\hline Monocorophium insidiosum & 0.0 & 0.0 & 0.0 & & & & \\
\hline Monocorophium unid. spp. & 0.0 & 0.0 & 0.0 & & & & \\
\hline Oedicerotidae unid spp. & 0.0 & 0.0 & 0.0 & & & & \\
\hline Order Cumacea & & & & & & & \\
\hline Nippoleucon hinumensis & 0.0 & 0.0 & 0.0 & & & & \\
\hline Order Decapoda & & & & & & & \\
\hline Crangon franciscorum & 0.0 & 0.0 & 0.0 & & & & \\
\hline Hemigrapsus oregonensis & 0.0 & 0.0 & 0.0 & & & & \\
\hline Crab unid spp. & 0.0 & 0.0 & 0.0 & & & & \\
\hline Order Isopoda & & & & & & & \\
\hline Gnorisphaeroma oregonensis & 0.0 & 0.0 & 0.0 & & & & \\
\hline Paranthura japonica & 0.0 & 0.0 & 0.0 & & & & \\
\hline Synidotea laevidorsalis & 0.0 & 0.0 & 0.0 & & & & \\
\hline Synidotea unid. spp. & 0.0 & 0.0 & 0.0 & & & & \\
\hline Class Ostracoda & & & & & & & \\
\hline Eusarsiella zostericola & 0.0 & 0.0 & 0.0 & & & & \\
\hline Cyprideis unid. spp. & 0.0 & 0.0 & 0.0 & & & & \\
\hline Class Thecostraca & & & & & & & \\
\hline Balanomorpha unid. spp. & 0.0 & 0.0 & 0.0 & & & & \\
\hline Cirripedia unid. spp. & 0.0 & 0.0 & 0.0 & & & & \\
\hline
\end{tabular}


Appendix 13. SDBGC2 Species List and Abundance

\begin{tabular}{|c|c|c|c|c|c|c|c|}
\hline TAXON & 28-Jan-14 & 27-Feb-14 & 27-Mar-14 & TAXON & 28-Jan-14 & 27-Feb-14 & 27-Mar-14 \\
\hline $\begin{array}{l}\text { PHYLUM ANNELIDA } \\
\text { Class Oligochaeta }\end{array}$ & & & & $\begin{array}{l}\text { PHYLUM BRYOZOA } \\
\text { Class Gymnolaemata }\end{array}$ & & & \\
\hline Naididae unid. spp. & 0.0 & 0.0 & 0.0 & Cheilostomatida unid. Spp. & 0.0 & tmtc & 0.0 \\
\hline Oligochaeta unid. spp. & 0.0 & 0.0 & 0.0 & PHYLUM CHORDATA & & & \\
\hline Tubificidae unid. spp. & 1.0 & 0.0 & 0.0 & Class Ascidiacea & & & \\
\hline Class Polychaeta & & & & Molgula mahattensis & 0.0 & 0.0 & 0.0 \\
\hline Capitella capitata complex & 0.0 & 0.0 & 0.0 & PHYLUM CNIDARIA & & & \\
\hline Cirratulidae unid. spp. & 0.0 & 0.0 & 0.0 & Class Anthozoa & & & \\
\hline Eteone fauchaldi & 0.0 & 0.0 & 0.0 & Actiniaria - attached & 51.0 & 10.0 & 52.0 \\
\hline Eteone lighti & 0.0 & 0.0 & 0.0 & Actiniaria - burrowing & $\begin{array}{l}51.0 \\
1.0\end{array}$ & 0.0 & 0.0 \\
\hline Eteone unid. spp. & 0.0 & 0.0 & 0.0 & Actiniaria unid. spp. & 0.0 & 0.0 & 0.0 \\
\hline Euchone limnicola & 3.0 & 0.0 & 1.0 & Class Hydrozoa & & & \\
\hline Euchone unid. spp. & 0.0 & 0.0 & 0.0 & Hydrozoa unid spp. & 0.0 & 0.0 & 0.0 \\
\hline Exogone lourei & 0.0 & 0.0 & 0.0 & PHYLUM MOLLUSCA & & & \\
\hline Glycera unid. spp. & 0.0 & 0.0 & 0.0 & $\begin{array}{l}\text { PHYYUM MOLLUSCA } \\
\text { Class Bivalvia }\end{array}$ & & & \\
\hline Glycinde armigera & 0.0 & 0.0 & 0.0 & Bivalvia unid. spp. & 0.0 & 0.0 & 0.0 \\
\hline Glycinde picta & 0.0 & 0.0 & 0.0 & $\begin{array}{l}\text { BIVaIvia unid. spp. } \\
\text { Gemma gemma }\end{array}$ & 2.0 & 1.0 & 0.0 \\
\hline Glycinde unid. sp. SF1 & 0.0 & 0.0 & 0.0 & Macoma petalum & 0.0 & 0.0 & 0.0 \\
\hline Glycinde unid. spp. & 0.0 & 0.0 & 0.0 & $\begin{array}{l}\text { Macoma petalum } \\
\text { Musculista senhousia }\end{array}$ & 0.0 & 0.0 & 2.0 \\
\hline Harmothoe imbricata & 0.0 & 0.0 & 1.0 & Mya arenaria & 0.0 & 0.0 & 0.0 \\
\hline Heteromastus filiformis & 1.0 & 2.0 & 3.0 & Potamocorbula amurensis & 0.0 & 1.0 & 0.0 \\
\hline Leitoscoloplos pugettensis & 0.0 & 0.0 & 0.0 & Theora lubrica & 1.0 & 0.0 & 0.0 \\
\hline Maldanidae unid. spp. & 0.0 & 0.0 & 0.0 & $\begin{array}{l}\text { Iheora lubrica } \\
\text { Venerupis philippinarum }\end{array}$ & $\begin{array}{l}1.0 \\
0.0\end{array}$ & 0.0 & 2.0 \\
\hline Marphysa sanguinea & 0.0 & 0.0 & 0.0 & $\begin{array}{l}\text { Venerupis philippinarum } \\
\text { Class Gastropoda }\end{array}$ & 0.0 & & \\
\hline Neanthes succinea & 7.0 & 2.0 & 23.0 & $\begin{array}{l}\text { Class Gastropoda } \\
\text { Crepidula unid. Spp. }\end{array}$ & 0.0 & 0.0 & 0.0 \\
\hline Polychaeta unid. spp. (1) & 0.0 & 0.0 & 0.0 & $\begin{array}{l}\text { Crepidula unid. Spp. } \\
\text { Gastropoda unid. sp. B }\end{array}$ & 0.0 & 0.0 & 0.0 \\
\hline Polychaeta unid. spp. (2) & 0.0 & 0.0 & 0.0 & $\begin{array}{l}\text { Gastropoda unid. sp. B } \\
\text { Ilyanassa obsoleta }\end{array}$ & $\begin{array}{l}0.0 \\
0.0\end{array}$ & $\begin{array}{l}0.0 \\
1.0\end{array}$ & 0.0 \\
\hline Polycirrus spp. & 0.0 & 0.0 & 0.0 & $\begin{array}{l}\text { Ilyanassa obsoleta } \\
\text { Philine unid. spp. }\end{array}$ & 0.0 & $\begin{array}{l}1.0 \\
0.0\end{array}$ & 0.0 \\
\hline Polydora cornuta & 0.0 & 0.0 & 0.0 & Urosalpinx cinerea & 0.0 & 0.0 & 0.0 \\
\hline Polydora unid. spp. & 0.0 & 0.0 & 0.0 & PHYLUM NEMATODA & & & \\
\hline Pseudopolydora kempi & 0.0 & 0.0 & 0.0 & Nematoda unid. spp. & 0.0 & 0.0 & 0.0 \\
\hline $\begin{array}{l}\text { Pseudopolydora paucibranchiata } \\
\text { Sabaco elongatus }\end{array}$ & $\begin{array}{l}0.0 \\
0.0\end{array}$ & $\begin{array}{l}0.0 \\
0.0\end{array}$ & $\begin{array}{l}0.0 \\
0.0\end{array}$ & PHYLUM PLATYHELMINTHES & & & \\
\hline Sabellidae unid. spp. & 0.0 & 0.0 & 0.0 & Class Turbellaria & & & \\
\hline Sphaerosyllis californiensis & 0.0 & 0.0 & 0.0 & Turbellaria unid. sp. A & 0.0 & 0.0 & 0.0 \\
\hline Sphaerosyllis erinaceus & 0.0 & 0.0 & 0.0 & Turbellaria unid. spp. & 0.0 & 0.0 & 0.0 \\
\hline Sphaerosyllis unid. sp. A & 0.0 & 0.0 & 0.0 & & & & \\
\hline Streblospio benedicti & 84.0 & 0.0 & 0.0 & & & & \\
\hline Spionidae unid. spp. & 0.0 & 0.0 & 0.0 & & & & \\
\hline $\begin{array}{l}\text { PHYLUM ARTHROPODA } \\
\text { Class Copepoda }\end{array}$ & & & & & & & \\
\hline Calanoida unid. spp. & 0.0 & 0.0 & 0.0 & & & & \\
\hline Harpacticoida unid. spp. & 0.0 & 0.0 & 0.0 & & & & \\
\hline $\begin{array}{l}\text { Class Malacostraca } \\
\text { Order Amphipoda }\end{array}$ & & & & & & & \\
\hline Ampelisca abdita & 614.0 & 0.0 & 0.0 & & & & \\
\hline Ampithoe unid. spp. & 0.0 & 0.0 & 0.0 & & & & \\
\hline Caprella californica & 0.0 & 0.0 & 0.0 & & & & \\
\hline Corophium heteroceratum & 49.0 & 0.0 & 521.0 & & & & \\
\hline Corophiidae unid. spp. & 0.0 & 0.0 & 0.0 & & & & \\
\hline Grandidierella japonica & 4.0 & 0.0 & 34.0 & & & & \\
\hline Monocorophium acherusicum & 0.0 & 0.0 & 0.0 & & & & \\
\hline Monocorophium insidiosum & 0.0 & 0.0 & 0.0 & & & & \\
\hline Monocorophium unid. spp. & 0.0 & 0.0 & 0.0 & & & & \\
\hline Oedicerotidae unid spp. & 0.0 & 0.0 & 0.0 & & & & \\
\hline Order Cumacea & & & & & & & \\
\hline Nippoleucon hinumensis & 0.0 & 0.0 & 0.0 & & & & \\
\hline Order Decapoda & & & & & & & \\
\hline Crangon franciscorum & 0.0 & 0.0 & 0.0 & & & & \\
\hline Hemigrapsus oregonensis & 0.0 & 0.0 & 0.0 & & & & \\
\hline Crab unid spp. & 0.0 & 0.0 & 0.0 & & & & \\
\hline Order Isopoda & & & & & & & \\
\hline Gnorisphaeroma oregonensis & 0.0 & 0.0 & 0.0 & & & & \\
\hline Paranthura japonica & 1.0 & 0.0 & 0.0 & & & & \\
\hline Synidotea laevidorsalis & 1.0 & 0.0 & 0.0 & & & & \\
\hline Synidotea unid. spp. & 0.0 & 0.0 & 0.0 & & & & \\
\hline Class Ostracoda & & & & & & & \\
\hline Eusarsiella zostericola & 0.0 & 0.0 & 0.0 & & & & \\
\hline Cyprideis unid. spp. & 0.0 & 0.0 & 0.0 & & & & \\
\hline Class Thecostraca & & & & & & & \\
\hline Balanomorpha unid. spp. & 0.0 & 0.0 & 0.0 & & & & \\
\hline Cirripedia unid. spp. & 0.0 & 0.0 & 0.0 & & & & \\
\hline
\end{tabular}


Appendix 13. SDBGC2 Species List and Abundance-Continued

\begin{tabular}{|c|c|c|c|c|c|c|c|}
\hline TAXON & 25-Apr-14 & 24-Jun-14 & 19-Aug-14 & TAXON & 25-Apr-14 & 24-Jun-14 & 19-Aug-14 \\
\hline $\begin{array}{l}\text { PHYLUM ANNELIDA } \\
\text { Class Oligochaeta }\end{array}$ & & & & $\begin{array}{l}\text { PHYLUM BRYOZOA } \\
\text { Class Gymnolaemata }\end{array}$ & & & \\
\hline Naididae unid. spp. & 0.0 & 0.0 & 0.0 & Cheilostomatida unid. Spp. & 0.0 & 0.0 & 0.0 \\
\hline Oligochaeta unid. spp. & 0.0 & 0.0 & 0.0 & PHYLUM CHORDATA & & & \\
\hline Tubificidae unid. spp. & 0.0 & 0.0 & 3.0 & Class Ascidiacea & & & \\
\hline Class Polychaeta & & & & Molgula mahattensis & 0.0 & 0.0 & 0.0 \\
\hline Capitella capitata complex & 0.0 & 0.0 & 0.0 & PHYLUM CNIDARIA & & & \\
\hline Cirratulidae unid. spp. & 0.0 & 0.0 & 2.0 & Class Anthozoa & & & \\
\hline Eteone fauchaldi & 1.0 & 0.0 & 1.0 & $\begin{array}{l}\text { Class Anthozoa } \\
\text { Actiniaria - attached }\end{array}$ & 0.0 & 1.0 & 66.0 \\
\hline Eteone lighti & 0.0 & 0.0 & 0.0 & Actiniaria - burrowing & 0.0 & 0.0 & 0.0 \\
\hline Eteone unid. spp. & 0.0 & 0.0 & 0.0 & Actiniaria unid. spp. & 0.0 & 0.0 & 0.0 \\
\hline Euchone limnicola & 0.0 & 0.0 & 1.0 & Class Hydrozoa & & & \\
\hline Euchone unid. spp. & 0.0 & 0.0 & 0.0 & Hydrozoa unid spp. & 0.0 & 0.0 & 0.0 \\
\hline Exogone lourei & 0.0 & 0.0 & 0.0 & PHYLUM MOLLUSCA & & & \\
\hline Glycera unid. spp. & 0.0 & 0.0 & 0.0 & $\begin{array}{l}\text { PHYLUM MOLLUSCA } \\
\text { Class Bivalvia }\end{array}$ & & & \\
\hline Glycinde armigera & 0.0 & 0.0 & 0.0 & Bivalvia unid. spp. & 0.0 & 0.0 & 0.0 \\
\hline Glycinde picta & 0.0 & 2.0 & 2.0 & $\begin{array}{l}\text { BIValvia unid. spp. } \\
\text { Gemma gemma }\end{array}$ & $\begin{array}{c}0.0 \\
14.0\end{array}$ & $\begin{array}{c}0.0 \\
467.0\end{array}$ & $\begin{array}{l}0.0 \\
86.0\end{array}$ \\
\hline Glycinde unid. sp. SF1 & 0.0 & 0.0 & 0.0 & $\begin{array}{l}\text { Gemma gemma } \\
\text { Macoma petalum }\end{array}$ & $\begin{array}{c}14.0 \\
1.0\end{array}$ & 1.0 & 2.0 \\
\hline Glycinde unid. spp. & 0.0 & 2.0 & 1.0 & Musculista senhousia & 0.0 & 0.0 & 0.0 \\
\hline Harmothoe imbricata & 1.0 & 0.0 & 2.0 & Mya arenaria & 0.0 & 3.0 & 0.0 \\
\hline Heteromastus filiformis & 0.0 & 0.0 & 27.0 & Potamocorbula amurensis & 0.0 & 1.0 & 1.0 \\
\hline Leitoscoloplos pugettensis & 0.0 & 0.0 & 1.0 & Theora lubrica & 0.0 & 0.0 & 0.0 \\
\hline Maldanidae unid. spp. & 0.0 & 0.0 & 0.0 & $\begin{array}{l}\text { Iheora lubrica } \\
\text { Venerupis philippinarum }\end{array}$ & 0.0 & 0.0 & 2.0 \\
\hline Marphysa sanguinea & 1.0 & 0.0 & 0.0 & $\begin{array}{l}\text { Venerupis philippinarum } \\
\text { Class Gastropoda }\end{array}$ & 0.0 & 0.0 & \\
\hline Neanthes succinea & 1.0 & 7.0 & 33.0 & $\begin{array}{l}\text { Class Gastropoda } \\
\text { Crepidula unid. Spp. }\end{array}$ & 0.0 & 0.0 & 0.0 \\
\hline Polychaeta unid. spp. (1) & 0.0 & 0.0 & 0.0 & $\begin{array}{l}\text { Crepidula unid. Spp. } \\
\text { Gastropoda unid. sp. B }\end{array}$ & 0.0 & 0.0 & 0.0 \\
\hline Polychaeta unid. spp. (2) & 0.0 & 0.0 & 0.0 & $\begin{array}{l}\text { Gastropoda unid. sp. B } \\
\text { Ilyanassa obsoleta }\end{array}$ & $\begin{array}{l}0.0 \\
0.0\end{array}$ & 0.0 & 0.0 \\
\hline Polycirrus spp. & 0.0 & 0.0 & 0.0 & $\begin{array}{l}\text { Ilyanassa obsoleta } \\
\text { Philine unid. spp. }\end{array}$ & $\begin{array}{l}0.0 \\
0.0\end{array}$ & 0.0 & 0.0 \\
\hline Polydora cornuta & 3.0 & 0.0 & 5.0 & Urosalpinx cinerea & 0.0 & 0.0 & 0.0 \\
\hline Polydora unid. spp. & 0.0 & 0.0 & 0.0 & PHYLUM NEMATODA & & & \\
\hline Pseudopolydora kempi & 0.0 & 0.0 & 0.0 & Nematoda unid. spp. & 0.0 & 0.0 & 0.0 \\
\hline $\begin{array}{l}\text { Pseudopolydora paucibranchiata } \\
\text { Sabaco elongatus }\end{array}$ & $\begin{array}{l}0.0 \\
0.0\end{array}$ & $\begin{array}{l}0.0 \\
0.0\end{array}$ & $\begin{array}{l}0.0 \\
0.0\end{array}$ & PHYLUM PLATYHELMINTHES & & & \\
\hline Sabellidae unid. spp. & 0.0 & 0.0 & 0.0 & Class Turbellaria & & & \\
\hline Sphaerosyllis californiensis & 0.0 & 0.0 & 0.0 & Turbellaria unid. sp. A & 0.0 & 0.0 & 0.0 \\
\hline Sphaerosyllis erinaceus & 0.0 & $\begin{array}{l}0.0 \\
0.0\end{array}$ & $\begin{array}{l}0.0 \\
0.0\end{array}$ & Turbellaria unid. spp. & 0.0 & 0.0 & 0.0 \\
\hline Sphaerosyllis unid. sp. A & 0.0 & 0.0 & 0.0 & & & & \\
\hline Streblospio benedicti & 0.0 & 0.0 & 74.0 & & & & \\
\hline Spionidae unid. spp. & 0.0 & 0.0 & 0.0 & & & & \\
\hline $\begin{array}{l}\text { PHYLUM ARTHROPODA } \\
\text { Class Copepoda }\end{array}$ & & & & & & & \\
\hline Calanoida unid. spp. & 0.0 & 0.0 & 0.0 & & & & \\
\hline Harpacticoida unid. spp. & 0.0 & 0.0 & 0.0 & & & & \\
\hline Class Malacostraca & & & & & & & \\
\hline Order Amphipoda & & & & & & & \\
\hline Ampelisca abdita & 0.0 & 69.0 & 31.0 & & & & \\
\hline Ampithoe unid. spp. & 0.0 & 0.0 & 0.0 & & & & \\
\hline Caprella californica & 0.0 & 0.0 & 0.0 & & & & \\
\hline Corophium heteroceratum & 158.0 & 0.0 & 0.0 & & & & \\
\hline Corophiidae unid. spp. & 0.0 & 0.0 & 0.0 & & & & \\
\hline Grandidierella japonica & 1.0 & 0.0 & 0.0 & & & & \\
\hline Monocorophium acherusicum & 0.0 & 0.0 & 2.0 & & & & \\
\hline Monocorophium insidiosum & 0.0 & 0.0 & 0.0 & & & & \\
\hline Monocorophium unid. spp. & 3.0 & 0.0 & 0.0 & & & & \\
\hline Oedicerotidae unid spp. & 0.0 & 0.0 & 0.0 & & & & \\
\hline Order Cumacea & & & & & & & \\
\hline Nippoleucon hinumensis & 2.0 & 0.0 & 0.0 & & & & \\
\hline Order Decapoda & & & & & & & \\
\hline Crangon franciscorum & 0.0 & 0.0 & 0.0 & & & & \\
\hline Hemigrapsus oregonensis & 0.0 & 0.0 & 0.0 & & & & \\
\hline Crab unid spp. & 0.0 & 0.0 & 0.0 & & & & \\
\hline Order Isopoda & & & & & & & \\
\hline Gnorisphaeroma oregonensis & 0.0 & 0.0 & 0.0 & & & & \\
\hline Paranthura japonica & 0.0 & 1.0 & 0.0 & & & & \\
\hline Synidotea laevidorsalis & 0.0 & 0.0 & 1.0 & & & & \\
\hline Synidotea unid. spp. & 0.0 & 0.0 & 0.0 & & & & \\
\hline Class Ostracoda & & & & & & & \\
\hline Eusarsiella zostericola & 0.0 & 0.0 & 1.0 & & & & \\
\hline Cyprideis unid. spp. & 0.0 & 0.0 & 0.0 & & & & \\
\hline Class Thecostraca & & & & & & & \\
\hline Balanomorpha unid. spp. & 0.0 & 0.0 & 0.0 & & & & \\
\hline Cirripedia unid. spp. & 0.0 & 0.0 & 0.0 & & & & \\
\hline
\end{tabular}


Appendix 13. SDBGC2 Species List and Abundance-Continued

\begin{tabular}{|c|c|c|c|c|c|c|c|}
\hline TAXON & 24-Sep-14 & 21-Oct-14 & 13-Nov-14 & TAXON & 24-Sep-14 & 21-Oct-14 & 13-Nov-14 \\
\hline $\begin{array}{l}\text { PHYLUM ANNELIDA } \\
\text { Class Oligochaeta }\end{array}$ & & & & $\begin{array}{l}\text { PHYLUM BRYOZOA } \\
\text { Class Gymnolaemata }\end{array}$ & & & \\
\hline Naididae unid. spp. & 0.0 & 0.0 & 0.0 & Cheilostomatida unid. Spp. & 0.0 & 0.0 & 0.0 \\
\hline Oligochaeta unid. spp. & 0.0 & 0.0 & 0.0 & PHYLUM CHORDATA & & & \\
\hline Tubificidae unid. spp. & 0.0 & 13.0 & 0.0 & Class Ascidiacea & & & \\
\hline Class Polychaeta & & & & Molgula mahattensis & 0.0 & 0.0 & 0.0 \\
\hline Capitella capitata complex & 0.0 & 0.0 & 0.0 & PHYLUM CNIDARIA & & & \\
\hline Cirratulidae unid. spp. & 0.0 & 0.0 & 0.0 & Class Anthozoa & & & \\
\hline Eteone fauchaldi & 0.0 & 0.0 & 0.0 & Actiniaria - attached & 12.0 & 11.0 & 9.0 \\
\hline Eteone lighti & 0.0 & 1.0 & 5.0 & Actiniaria - burrowing & 0.0 & 0.0 & 0.0 \\
\hline Eteone unid. spp. & 0.0 & 0.0 & 0.0 & Actiniaria unid. spp. & 0.0 & 0.0 & 0.0 \\
\hline Euchone limnicola & 0.0 & 10.0 & 5.0 & Class Hydrozoa & & & \\
\hline Euchone unid. spp. & 0.0 & 0.0 & 0.0 & Hydrozoa unid spp. & 0.0 & 0.0 & 0.0 \\
\hline Exogone lourei & 0.0 & 0.0 & 1.0 & PHYLUM MOLLUSCA & & & \\
\hline Glycera unid. spp. & 0.0 & 0.0 & 0.0 & Class Bivalvia & & & \\
\hline Glycinde armigera & 0.0 & 0.0 & 0.0 & Bivalvia unid. spp. & 0.0 & 0.0 & 0.0 \\
\hline Glycinde picta & 0.0 & 0.0 & 2.0 & Gemma gemma & 271.0 & 45.0 & 0.0 \\
\hline Glycinde unid. sp. SF1 & 0.0 & 0.0 & 0.0 & Macoma petalum & 0.0 & 0.0 & 0.0 \\
\hline Glycinde unid. spp. & 0.0 & 0.0 & 0.0 & Musculista senhousia & 1.0 & 0.0 & 0.0 \\
\hline Harmothoe imbricata & 0.0 & 0.0 & 0.0 & Mya arenaria & 0.0 & 0.0 & 0.0 \\
\hline Heteromastus filiformis & 0.0 & 15.0 & 25.0 & Potamocorbula amurensis & 0.0 & 1.0 & 0.0 \\
\hline Leitoscoloplos pugettensis & 0.0 & 0.0 & 0.0 & Theora lubrica & 0.0 & 0.0 & 0.0 \\
\hline Maldanidae unid. spp. & 0.0 & 0.0 & 0.0 & Venerupis philippinarum & 1.0 & 0.0 & 0.0 \\
\hline Marphysa sanguinea & 0.0 & 0.0 & 15.0 & Class Gastropoda & 1.0 & 0.0 & \\
\hline Neanthes succinea & 0.0 & 41.0 & 17.0 & Crepidula unid. Spp. & 0.0 & 0.0 & 0.0 \\
\hline Polychaeta unid. spp. (1) & 1 vial & 0.0 & 0.0 & Gastropoda unid. sp. B & 0.0 & 0.0 & 0.0 \\
\hline Polychaeta unid. spp. (2) & 0.0 & 0.0 & 0.0 & Ilyanassa obsoleta & 0.0 & 0.0 & 0.0 \\
\hline Polycirrus spp. & 0.0 & 0.0 & 0.0 & Philine unid. spp. & 0.0 & 0.0 & 0.0 \\
\hline Polydora cornuta & 0.0 & 0.0 & 0.0 & Urosalpinx cinerea & 0.0 & 0.0 & 0.0 \\
\hline Polydora unid. spp. & 0.0 & 0.0 & 0.0 & PHYLUM NEMATODA & & & \\
\hline Pseudopolydora kempi & 0.0 & 0.0 & 0.0 & Nematoda unid. spp. & 0.0 & 0.0 & 0.0 \\
\hline Pseudopolydora paucibranchiata & 0.0 & 0.0 & 0.0 & PHYLUM PLATYHELMINTHES & & & \\
\hline Sabaco elongatus & 0.0 & 0.0 & 0.0 & Class Turbellaria & & & \\
\hline Sabellidae unid. spp. & 0.0 & 0.0 & 0.0 & Turbellaria unid. sp. A & 0.0 & 0.0 & \\
\hline Sphaerosyllis californiensis & 0.0 & 0.0 & 0.0 & Turbellaria unid. spp. & 0.0 & 0.0 & 0.0 \\
\hline $\begin{array}{l}\text { Sphaerosyllis erinaceus } \\
\text { Sphaerosyllis unid. sp. A }\end{array}$ & $\begin{array}{l}0.0 \\
0.0\end{array}$ & $\begin{array}{l}0.0 \\
0.0\end{array}$ & $\begin{array}{l}0.0 \\
0.0\end{array}$ & & & & \\
\hline Streblospio benedicti & 0.0 & 66.0 & 259.0 & & & & \\
\hline Spionidae unid. spp. & 0.0 & 0.0 & 0.0 & & & & \\
\hline PHYLUM ARTHROPODA & & & & & & & \\
\hline Class Copepoda & & & & & & & \\
\hline Calanoida unid. spp. & 0.0 & 0.0 & 0.0 & & & & \\
\hline Harpacticoida unid. spp. & 0.0 & 0.0 & 0.0 & & & & \\
\hline Class Malacostraca & & & & & & & \\
\hline Order Amphipoda & & & & & & & \\
\hline Ampelisca abdita & 121.0 & 49.0 & 7.0 & & & & \\
\hline Ampithoe unid. spp. & 0.0 & 0.0 & 0.0 & & & & \\
\hline Caprella californica & 0.0 & 0.0 & 0.0 & & & & \\
\hline Corophium heteroceratum & 144.0 & 4.0 & 16.0 & & & & \\
\hline Corophiidae unid. spp. & 0.0 & 0.0 & 0.0 & & & & \\
\hline Grandidierella japonica & 0.0 & 0.0 & 0.0 & & & & \\
\hline Monocorophium acherusicum & 0.0 & 0.0 & 0.0 & & & & \\
\hline Monocorophium insidiosum & 0.0 & 0.0 & 0.0 & & & & \\
\hline Monocorophium unid. spp. & 0.0 & 0.0 & 0.0 & & & & \\
\hline Oedicerotidae unid spp. & 0.0 & 0.0 & 0.0 & & & & \\
\hline Order Cumacea & & & & & & & \\
\hline Nippoleucon hinumensis & 0.0 & 0.0 & 0.0 & & & & \\
\hline Order Decapoda & & & & & & & \\
\hline Crangon franciscorum & 0.0 & 0.0 & 0.0 & & & & \\
\hline Hemigrapsus oregonensis & 0.0 & 0.0 & 0.0 & & & & \\
\hline $\begin{array}{l}\text { Crab unid spp. } \\
\text { Order Isopoda }\end{array}$ & 0.0 & 0.0 & 0.0 & & & & \\
\hline Gnorisphaeroma oregonensis & 0.0 & 0.0 & 0.0 & & & & \\
\hline Paranthura japonica & 2.0 & 3.0 & 0.0 & & & & \\
\hline Synidotea laevidorsalis & 0.0 & 0.0 & 0.0 & & & & \\
\hline Synidotea unid. spp. & 0.0 & 0.0 & 0.0 & & & & \\
\hline Class Ostracoda & & & & & & & \\
\hline Eusarsiella zostericola & 0.0 & 0.0 & 0.0 & & & & \\
\hline Cyprideis unid. spp. & 0.0 & 0.0 & 0.0 & & & & \\
\hline Class Thecostraca & & & & & & & \\
\hline Balanomorpha unid. spp. & 0.0 & 0.0 & 0.0 & & & & \\
\hline Cirripedia unid. spp. & 0.0 & 0.0 & 0.0 & & & & \\
\hline
\end{tabular}




\section{Appendix 14. AVS Bivalve Biomass}

STATION: AVS1

\begin{tabular}{l|ccc}
\hline \multicolumn{1}{c|}{ Date } & Macoma petalum & Mya arenaria & Potamocorbula amurensis \\
\hline August 6, 2013 & 5.047 & 0.626 & 16.065 \\
March 27, 2014 & 10.101 & 0.239 & 0.000 \\
June 24 2014 & 13.780 & 0.000 & 5.820 \\
September 24,2014 & 6.220 & 0.000 & 8.098 \\
\hline
\end{tabular}

STATION: AVS2

\begin{tabular}{l|ccc}
\hline \multicolumn{1}{c|}{ Date } & Macoma petalum & Mya arenaria & Potamocorbula amurensis \\
\hline August 6, 2013 & 34.353 & 0.000 & 5.256 \\
March 27, 2014 & 171.334 & 1.412 & 1.904 \\
June 24 2014 & 5.572 & 0.000 & 14.157 \\
September 24,2014 & 12.841 & 0.000 & 26.954 \\
\hline
\end{tabular}

STATION: AVS3

\begin{tabular}{l|ccc}
\hline \multicolumn{1}{c|}{ Date } & Macoma petalum & Mya arenaria & Potamocorbula amurensis \\
\hline August 6, 2013 & 34.353 & 0.000 & 5.256 \\
March 27, 2014 & 0.000 & 0.000 & 0.000 \\
June 24 2014 & 88.669 & 6.127 & 0.814 \\
September 24,2014 & 11.882 & 5.235 & 5.336 \\
\hline
\end{tabular}




\section{Appendix 15. GPE Bivalve Biomass}

STATION: GPE1

\begin{tabular}{lccc}
\hline \multicolumn{1}{c}{ Date } & Macoma petalum & Mya arenaria & Potamocorbula amurensis \\
\hline August 6, 2013 & 9.440 & 0.003 & 15.937 \\
March 27, 2014 & 6.265 & 0.000 & 0.771 \\
June 24 2014 & 2.379 & 0.000 & 5.580 \\
September 24,2014 & 0.215 & 0.000 & 5.416 \\
\hline
\end{tabular}

STATION: GPE2

\begin{tabular}{lccc}
\hline \multicolumn{1}{c}{ Date } & Macoma petalum & Mya arenaria & Potamocorbula amurensis \\
\hline August 6, 2013 & 6.967 & 0.000 & 4.238 \\
March 27, 2014 & 1.358 & 0.000 & 0.242 \\
June 24 2014 & 2.921 & 1.325 & 3.921 \\
September 24,2014 & 8.624 & 1.193 & 3.973 \\
\hline
\end{tabular}

STATION: GPE3

\begin{tabular}{lccc}
\hline \multicolumn{1}{c}{ Date } & Macoma petalum & Mya arenaria & Potamocorbula amurensis \\
\hline August 6, 2013 & 8.988 & 0.284 & 24.366 \\
March 27, 2014 & 3.301 & 0.000 & 0.154 \\
June 24 2014 & 11.226 & 0.000 & 10.818 \\
September 24,2014 & 11.497 & 1.463 & 8.615 \\
\hline
\end{tabular}

STATION: GPE4

\begin{tabular}{lccc}
\hline \multicolumn{1}{c}{ Date } & Macoma petalum & Mya arenaria & Potamocorbula amurensis \\
\hline August 6, 2013 & 19.332 & 0.000 & 7.696 \\
March 27, 2014 & 0.040 & 0.000 & 0.081 \\
June 24 2014 & 15.013 & 0.000 & 0.368 \\
September 24,2014 & 0.000 & 0.000 & 0.000 \\
\hline
\end{tabular}




\section{Appendix 16. SB Bivalve Biomass}

\section{STATION: SB13}

\begin{tabular}{lccc}
\hline \multicolumn{1}{c}{ Date } & Macoma petalum & Mya arenaria & Potamocorbula amurensis \\
\hline March 27, 2014 & 0.576 & 0.000 & 0.000 \\
June 24 2014 & 0.138 & 0.000 & 0.000 \\
September 24,2014 & 1.912 & 0.000 & 0.000 \\
\hline \multicolumn{4}{c}{ STATION: SB14 } \\
\multicolumn{1}{c}{ Date } & Macoma petalum & Mya arenaria & Potamocorbula amurensis \\
\hline March 27, 2014 & 0.000 & 0.000 & 0.744 \\
June 24 2014 & 0.000 & 0.000 & 0.494 \\
September 24,2014 & 0.000 & 0.000 & 0.000 \\
\hline
\end{tabular}


Appendix 17. CC1 Bivalve Biomass

STATION: CC1

\begin{tabular}{|c|c|c|c|}
\hline Date & Macoma petalum & Mya arenaria & Potamocorbula amurensis \\
\hline 15-Oct-09 & 0.000 & 0.000 & 5.186 \\
\hline 10-Nov-09 & 1.043 & 0.357 & 0.710 \\
\hline 10-Feb-10 & 0.000 & 0.000 & 0.168 \\
\hline 16-Mar-10 & 3.013 & 0.000 & 1.392 \\
\hline 8-Apr-10 & 2.345 & 0.000 & 1.552 \\
\hline 11-Мay-10 & 1.218 & 0.000 & 0.284 \\
\hline 7-Jul-10 & 0.000 & 0.000 & 4.208 \\
\hline 9-Nov-10 & 0.408 & 0.000 & 20.112 \\
\hline 19-Jan-11 & 0.000 & 0.000 & 8.709 \\
\hline 15-Mar-11 & 0.215 & 0.000 & 3.105 \\
\hline 27-Apr-11 & 0.000 & 0.000 & 2.714 \\
\hline 23-Jun-11 & 0.000 & 0.000 & 1.802 \\
\hline 7-Sep-11 & 0.994 & 0.000 & 55.581 \\
\hline 5-Oct-11 & 0.795 & 0.000 & 41.096 \\
\hline 2-Nov-11 & 0.000 & 0.000 & 13.032 \\
\hline 3-Apr-12 & 0.000 & 0.000 & 161.974 \\
\hline 13-Jun-12 & 0.000 & 0.000 & 27.660 \\
\hline 10-Jul-12 & 0.000 & 0.000 & 13.690 \\
\hline 6-Sep-12 & 1.290 & 0.000 & 52.578 \\
\hline 11-Oct-12 & 2.912 & 0.000 & 40.534 \\
\hline 15-Nov-12 & 0.590 & 0.000 & 29.044 \\
\hline 10-Jan-13 & 2.010 & 11.302 & 10.967 \\
\hline 7-Мау-13 & 1.127 & 0.335 & 4.743 \\
\hline 4-Jun-13 & 4.665 & 4.091 & 5.278 \\
\hline 1-Aug-13 & 4.342 & 1.760 & 10.396 \\
\hline 4-Sep-13 & 0.319 & 3.071 & 26.366 \\
\hline 5-Nov-13 & 3.268 & 0.000 & 1.578 \\
\hline 28-Jan-14 & 2.317 & 0.000 & 1.730 \\
\hline 27-Feb-14 & 0.078 & 0.000 & 0.968 \\
\hline 27-Mar-14 & 0.000 & 0.000 & 0.263 \\
\hline 25-Apr-24 & 1.089 & 0.141 & 9.771 \\
\hline 24-Jun-14 & 0.000 & 2.542 & 9.170 \\
\hline 19-Aug-14 & 0.684 & 0.000 & 10.961 \\
\hline 24-Sep-14 & 0.000 & 0.000 & 0.733 \\
\hline 21-Oct-14 & 0.454 & 0.000 & 5.333 \\
\hline 13-Nov-14 & 0.000 & 0.000 & 0.926 \\
\hline 18-Dec-14 & 0.531 & 0.000 & 1.495 \\
\hline
\end{tabular}




\section{Appendix 18. D3 Bivalve Biomass}

STATION: D3

\begin{tabular}{|c|c|c|c|}
\hline Date & Macoma petalum & Mya arenaria & Potamocorbula amurensis \\
\hline 10-Mar-09 & 4.475 & 0.000 & 0.440 \\
\hline 30-Jun-09 & 1.807 & 0.119 & 4.563 \\
\hline 11-Aug-09 & 5.093 & 1.372 & 65.179 \\
\hline 15-Oct-09 & 1.000 & 0.000 & 9.082 \\
\hline 10-Nov-09 & 2.500 & 0.000 & 0.000 \\
\hline 10-Feb-10 & 0.987 & 1.113 & 0.529 \\
\hline 16-Mar-10 & 2.540 & 0.572 & 0.284 \\
\hline 11-May-10 & 0.340 & 0.000 & 0.323 \\
\hline 7-Jul-10 & 3.589 & 0.000 & 15.473 \\
\hline 9-Nov-10 & 2.112 & 0.000 & 67.213 \\
\hline 19-Jan-11 & 0.424 & 0.000 & 0.000 \\
\hline 15-Mar-11 & 0.134 & 0.496 & 0.183 \\
\hline 27-Apr-11 & 0.716 & 0.000 & 0.226 \\
\hline 23-Jun-11 & 2.584 & 0.000 & 19.952 \\
\hline 7-Sep-11 & 7.546 & 0.000 & 2.111 \\
\hline 5-Oct-11 & 4.189 & 0.000 & 49.063 \\
\hline 2-Nov-11 & 4.343 & 0.667 & 116.649 \\
\hline 3-Apr-12 & 1.648 & 0.000 & 3.210 \\
\hline 13-Jun-12 & 8.159 & 0.905 & 28.620 \\
\hline 10-Jul-12 & 2.254 & 0.850 & 94.867 \\
\hline 6-Sep-12 & 3.412 & 0.558 & 268.418 \\
\hline 11-Oct-12 & 6.539 & 7.715 & 134.960 \\
\hline 15-Nov-12 & 8.601 & 7.567 & 40.913 \\
\hline 10-Jan-13 & 0.000 & 0.008 & 1.954 \\
\hline 7-Мay-13 & 4.777 & 10.680 & 8.164 \\
\hline 4-Jun-13 & 3.227 & 7.088 & 6.804 \\
\hline 1-Aug-13 & 9.939 & 3.010 & 23.612 \\
\hline 4-Sep-13 & 16.512 & 0.000 & 11.894 \\
\hline 5-Nov-13 & 4.497 & 11.184 & 3.608 \\
\hline 28-Jan-14 & 3.540 & 0.308 & 0.740 \\
\hline 27-Mar-14 & 0.184 & 0.000 & 0.000 \\
\hline 25-Apr-14 & 6.859 & 0.153 & 2.215 \\
\hline 24-Jun-14 & 4.610 & 0.016 & 7.102 \\
\hline 19-Aug-14 & 2.343 & 0.996 & 3.778 \\
\hline 24-Sep-14 & 0.057 & 0.000 & 1.522 \\
\hline 21-Oct-14 & 2.580 & 0.000 & 3.357 \\
\hline 13-Nov-14 & 4.670 & 0.000 & 0.888 \\
\hline 18-Dec-14 & 1.716 & 0.000 & 0.304 \\
\hline
\end{tabular}

